\title{
Discovering Evolving Temporal Information: Theory and Application to Clinical Databases
}

\author{
Pietro Sala ${ }^{1} \cdot$ Carlo Combi $^{1} \cdot$ Matteo Mantovani $^{1} \cdot$ Romeo Rizzi $^{1}$
}

Received: 10 March 2020 / Accepted: 9 April 2020 / Published online: 8 May 2020

(c) The Author(s) 2020

\begin{abstract}
Functional dependencies (FDs) allow us to represent database constraints, corresponding to requirements as "patients having the same symptoms undergo the same medical tests." Some research efforts have focused on extending such dependencies to consider also temporal constraints such as "patients having the same symptoms undergo in the next period the same medical tests." Temporal functional dependencies are able to represent such kind of temporal constraints in relational databases. Another extension for FDs allows one to represent approximate functional dependencies (AFDs), as "patients with the same symptoms generally undergo the same medical tests." It enables data to deviate from the defined constraints according to a user-defined percentage. Approximate temporal functional dependencies (ATFDs) merge the concepts of temporal functional dependency and of approximate functional dependency. Among the different kinds of ATFD, the Approximate Pure Temporally Evolving Functional Dependencies (APE-FDs for short) allow one to detect patterns on the evolution of data in the database and to discover dependencies as "For most patients with the same initial diagnosis, the same medical test is prescribed after the occurrence of same symptom." Mining ATFDs from large databases may be computationally expensive. In this paper, we focus on $A P E$-FDs and prove that, unfortunately, verifying a single $A P E$-FD over a given database instance is in general NP-complete. In order to cope with this problem, we propose a framework for mining complex APE-FDs in real-world data collections. In the framework, we designed and applied sound and advanced model-checking techniques. To prove the feasibility of our proposal, we used real-world databases from two medical domains (namely, psychiatry and pharmacovigilance) and tested the running prototype we developed on such databases.
\end{abstract}

Keywords Temporal data mining · Temporal functional dependencies $\cdot$ Temporal databases · Distributed algorithms · Complexity $\cdot$ Pharmacovigilance $\cdot$ Psychiatric case register

\section{Introduction}

Since some decades, in most of the real-world domains, there is the need of storing and analyzing huge and often overwhelming quantities of data, which are required both for decision making and in the wider area of the management of

Carlo Combi

carlo.combi@univr.it

Pietro Sala

pietro.sala@univr.it

Matteo Mantovani

matteo.mantovani@univr.it

Romeo Rizzi

romeo.rizzi@univr.it

1 Department of Computer Science, University of Verona, Verona, Italy complex organizations $[23,29]$. According to this scenario, and without loss of generality, in this paper, we will focus on the healthcare/medical domain, where such need arises, to support clinical decision-making and healthcare policies [3]. Advanced techniques such as data mining and analysis allow medical stakeholders to extract useful knowledge from these data. In particular, it is often the case that such knowledge is inherently temporal, as it is discovered when analyzing data evolution, time series, and changes of information over time. Temporal data mining is the research area focusing on the analysis and discovery from data having some specific temporal characterization [8, 9, 25].

Considering data stored according to the well-known relational model, functional dependencies (FDs) are usually specified for expressing constraints on data and for improving the quality of database schemata, by deriving normal forms [2]. However, functional dependencies (FDs) 
could be used to derive some knowledge about the given database. As an example, let us consider a simple relation describing the adverse drug reactions patients may have during a hospitalization. Such a relation stores patient demographic data, together with drugs taken and the adverse reactions possibly occurring. Moreover, a temporal attribute time-stamps the adverse reactions. Typically, patients taking the same drug may have the same adverse reaction. Thus, we can derive a functional dependency between the patient drug and the adverse reaction. It may also be that such functional dependencies hold on "most tuples," but not on all of them. Such dependencies have been named approximate functional dependency (AFD) $[13,16]$. As an example, if we consider patients affected by some allergies that make unpredictable the reaction to a drug, the corresponding tuples will likely differ from all the other ones as for the dependency between drugs and adverse reactions.

Finer constraints may also be discovered. As for adverse drug reactions, for example, further drug prescriptions may follow, to mitigate these known effects. For example, suppose that some drugs are prescribed just to mitigate some wellknown adverse reaction. Thus, the drug prescribed just after a given adverse reaction will be related both to the adverse reaction and to the drug previously taken by patients. In such a case, prescribed drugs and related adverse reactions determine drugs administered next. We call this dependency a temporal functional dependency (TFD).

Approximate functional dependencies have been extensively considered, and some tools have been proposed for deriving such dependencies $[13,14,16,19]$. On the other side, temporal functional dependencies have been proposed according to different perspectives and considering different kinds of temporal features [7, 15, 30-32]. To the best of our knowledge, only some recent studies focused on approximate temporal functional dependencies $[4,5,11,26]$.

In this paper, we continue such studies by considering a different kind of approximate TFD and its application to data from clinical domains. More specifically, we will adopt the framework for temporal functional dependencies proposed by Combi et al. in [7], which allows the specification of multiple kinds of temporal functional dependencies. According to this framework, we consider here the issue of mining (approximate) temporal functional dependencies based on tuple temporal evolution. Temporal evolution of tuples has been originally proposed by Vianu [30] for the characterization of dynamic functional dependencies (DFDs), which allow one to specify constraints on the evolution of tuples in consecutive snapshots of a temporal database. Here, we consider the characterization of DFDs introduced in [7], called Pure Temporally Evolving TFDs ( $P E$-FDs). In particular, we consider the problem of extracting all Approximate $P E$-FDs, called APE-FDs, from a given temporal medical database.
Before moving to the more experimental side of our work, we provide a "negative," yet interesting, result about the complexity of checking APE-FDs. First, we prove that checking a single $A P E$-FD against a database instance is $N P$-Complete in the size of the instance (i.e., data complexity). Moreover, we noticed that the $N P$-completeness of this problem heavily relies on instances that are fictitious and imply properties of data that are unreasonable in many contexts such as the clinical one. We thus came out with a series of optimizations and heuristics that improve the performances with respect to the more general problem of checking an APE-FD against a database instance.

As we pointed out, mining APE-FDs introduces many computational challenges that require techniques inherited from different fields of Computer Science (e.g., model checking and combinatorial optimization). We embedded such techniques in a framework that has been implemented as a running prototype and applied to data from pharmacovigilance and psychiatry domains. With respect to the preliminary results presented in [10, 27], we focus here only on $A P E$ -FDs and do not consider the related temporal association rules $[10,27]$. We propose here a new, stronger and more focused definition of $P E-\mathrm{FD}$ and of the related $A P E-\mathrm{FD}$, by introducing also a bounded version of temporal evolution of data. Moreover, we provide a detailed discussion and proof of our theoretical results, by introducing a significantly improved and extended presentation with new and more complete examples. Furthermore, we introduce a completely new section, in which we propose a couple of novel optimization techniques for solving the problem of checking APE-FDs.

In the following, "Background and Related Work" section describes the background and the related work. "Discovering Pure Temporally Evolving Functional Dependencies" section formally introduces the concepts of $P E-F D$ and $A P E-F D$. "Some Motivating Clinical Scenarios" section introduces some motivating clinical scenarios using $P E$-FDs and $A P E$ -FDs. "The Computational Complexity of Checking $A P E$ FD" section proves the $N P$-Hardness of checking an APE-FD against a given temporal database. "Algorithms for Checking $A P E$-FDs" section provides a description of the algorithm that checks a single $A P E-F D$ against a given database plus a series of optimizations and heuristics that may be generally implemented in order to speed up such verification process. "Mining APE-FDs" section provides a high-level description of the main features of our prototype for mining such dependencies and the main ideas underlying its implementation; then, it provides interesting mined APE-FDs from the psychiatry and pharmacovigilance domains; in the last part of this section, we analyze the performances of the implemented prototype. "Conclusions" section draws some conclusions and sketches possible directions for future research. 


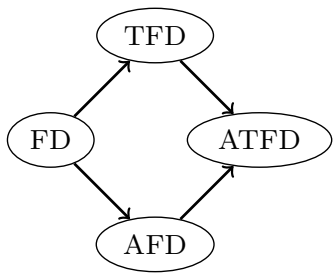

Fig. 1 A graphical account for the IS_A relationships between functional dependency (FD), approximate functional dependency (AFD), temporal functional dependency (TFD) and approximate temporal functional dependency (ATFD)

\section{Background and Related Work}

In this section, we introduce and discuss the main definitions and concepts we will use through this paper. We first recall the definition of functional dependency (FD). Then, we introduce some extensions of FDs, i.e., temporal functional dependencies (TFDs) and approximate functional dependencies (AFDs). The definition of approximate temporal functional dependency (ATFD) is grounded on these concepts. Figure 1 depicts the relationships among such kinds of functional dependencies.

\section{Functional Dependencies and Their Temporal Extensions}

The concept of functional dependency (FD) comes from the database theory [2].

Definition 1 Let $\mathbf{r}$ be a relation with schema $R$. Let $X, Y$ be sets of attributes of $R$. A functional dependency between $X$ and $Y$

$\mathbf{r} \vDash X \rightarrow Y$

represents the constraint that for all couples of tuples $t$ and $t^{\prime}$ in having the same value(s) on attribute(s) $X$, the corresponding value(s) on $Y$ for those tuples are identical.

More formally, relation $\mathbf{r}$ satisfies functional dependency $X \rightarrow Y$ if the following condition holds:

$\forall t, t^{\prime} \in \mathbf{r}\left(t[X]=t^{\prime}[X] \rightarrow t[Y]=t^{\prime}[Y]\right)$

Temporal functional dependencies (TFDs) have been proposed as extensions of (atemporal) functional dependencies [33]. As an example, we may represent the constraint that a pathology functionally determines a corresponding drug, but only considers tuples month by month. In other words, the patients with the same pathology are treated with a common drug during some month, while in another month the same patients affected by the same pathology take another (common) drug. Combi et al. proposed a framework for TFDs that subsumes and extends previous proposals [7]. They use a temporal relational data model, allowing one to represent the notion of temporal relation. Each relation is equipped with a time-stamping temporal attribute $\mathrm{VT}$, which represents the valid time, i.e., the time when the fact is true in the represented real world [6]. VT has values in domain $\mathcal{T}$ isomorphic to $\mathbb{N}$.

Two temporal views allow joining tuples that satisfy specific temporal conditions, which represent relevant cases of (temporal) evolution. On the basis of the introduced data model, and leveraging such temporal views, we may provide a definition for TFDs.

Definition 2 Let $R=U \cup\{V T\}$ be a relational schema where attributes in $U$ are atemporal and $V T$ has domain $\mathcal{T}$. A TFD is expressed as

\section{$[E-\operatorname{Exp}(R), t-$ Group $] X \rightarrow Y$}

where $E-\operatorname{Exp}(R)$ is a relational expression on schema $R$, called evolution expression, $t$ - Group is a mapping $\mathcal{T} \rightarrow 2^{\mathcal{T}}$, called temporal grouping, and $X \rightarrow Y$ is a functional dependency on the (atemporal) attributes $U$ of $E$ - $\operatorname{Exp}(R)$.

A TFD is a statement about admissible temporal relations. A temporal relation $\mathbf{r}$ on a temporal schema $R=U \cup\{V T\}$ satisfies a TFD $[E-\operatorname{Exp}(R), t$ - Group $] X \rightarrow Y$, if it is not possible that the relation obtained from $\mathbf{r}$ by applying the expression $E-\operatorname{Exp}(R)$ features two tuples $t, t^{\prime}$ such that

1. $t[X]=t^{\prime}[X]$,

2. $t[V T]$ and $t^{\prime}[V T]$ (the same for $t[\overline{V T}]$ and $t^{\prime}[\overline{V T}]$, if attribute $V T$, obtained by renaming $V T$, appears in the relation resulting from the evolution expression) belong to the same temporal group, according to the mapping $t$ - Group,

3. $t[Y] \neq t^{\prime}[Y]$.

In other words, FD $X \rightarrow Y$ must be satisfied by each relation obtained from the evolution expression by selecting those tuples whose valid times belong to the same temporal group. Temporal grouping enables us to group tuples together over a set of temporal granules, based on VT. Four different classes of TFD have been proposed in [7]:

- Pure temporally grouping TFD: $E$ - $\operatorname{Exp}(R)$ returns the original temporal relation $\mathbf{r}$. These TFDs force FD $X \rightarrow Y$, where $X, Y \subseteq U$, to hold over each set of tuples temporally grouped according to their $\mathrm{VT}$;

- Pure temporally evolving TFD: $E$ - $\operatorname{Exp}(R)$ specifies how to derive the tuples modeling the evolution of objects. No temporal grouping exists, i.e., all the tuples of $\mathbf{r}$ are considered together; 
- Temporally mixed TFD: in this case, after evaluating the expression $E$ - $\operatorname{Exp}(R)$, a temporal grouping $t$-Group is performed;

- Temporally hybrid TFD $s$ : first, the evolution expression $\operatorname{E-} \operatorname{Exp}(R)$ allows the selection of those tuples that are needed to represent the evolution of real-world objects/ concepts; then, temporal grouping is applied to the selected tuples.

In the remainder of the paper, we shall focus on Pure Temporally Evolving TFDs only.

\section{Approximate Functional Dependencies and Their Temporal Extensions}

The concept of approximate functional dependency (AFD) is defined moving from the concept of plain FD. In fact, given a relation $\mathbf{r}$ where an FD holds for most tuples in $\mathbf{r}$, we may identify some tuples, for which that FD does not hold. Consequently, we can define some measurements of the error we make in considering the FD to hold on $\mathbf{r}$.

One measurement [16] we can apply is known as $G_{1}$, which considers the number of violating pairs of tuples. Formally:

$G_{1}(X \rightarrow Y, \mathbf{r})=\left|\left\{\left(t, t^{\prime}\right): t, t^{\prime} \in \mathbf{r} \wedge t[X]=t^{\prime}[X] \wedge t[Y] \neq t^{\prime}[Y]\right\}\right|$

The related scaled measurement $g_{1}$ is defined as follows:

$g_{1}(X \rightarrow Y, \mathbf{r})=G_{1}(X \rightarrow Y, \mathbf{r}) /|\mathbf{r}|^{2}$

where $|r|$ is the cardinality of relation $\mathbf{r}$, i.e., the number of tuples belonging to relation $\mathbf{r}$.

Another measurement [16] we can apply is known as $G_{2}$, which considers the number of tuples that violate the functional dependency. Formally:

$$
\begin{aligned}
G_{2}(X \rightarrow Y, \mathbf{r}) & =\mid\left\{t: t \in \mathbf{r} \wedge \exists t^{\prime}\left(t^{\prime} \in \mathbf{r} \wedge t[X]\right.\right. \\
& \left.\left.=t^{\prime}[X] \wedge t[Y] \neq t^{\prime}[Y]\right)\right\} \mid
\end{aligned}
$$

The related scaled measurement $g_{2}$ is defined as follows:

$g_{2}(X \rightarrow Y, \mathbf{r})=G_{2}(X \rightarrow Y, \mathbf{r}) /|\mathbf{r}|$

Topics related to approximate functional dependencies have been considered since some years [13, 14, 16, 19]. Instead, to the best of our knowledge, very few studies focused on approximate temporal functional dependencies $[4,5,11$, 26]. In [4], Combi et al. consider the problem of mining approximate TFDs with different kinds of temporal grouping on clinical data. In $[11,26]$, Sala and Combi extend the concept of approximate TFD to deal with interval-based TFDs. In this paper, we continue such studies by considering a different kind of approximate TFD and its application to data from clinical domains.

\section{Discovering Pure Temporally Evolving Functional Dependencies}

In the following, we focus on Pure Temporally Evolving Functional Dependencies (PE-FDs for short), as specified in the framework proposed in [7]. Our temporal functional dependencies will be given on a temporal schema $R=U \cup\{V T\}$ where $U$ is a set of atemporal attributes and $V T$ is a special attribute denoting the valid time of each tuple. Hereinafter, we assume tuples time-stamped with natural numbers (i.e., $\operatorname{Dom}(V T)=\mathbb{N}$ ). Let $J \subseteq U$ be a nonempty subset of $U$. We define the set $W$ as $W=U \backslash J$ and set $\bar{W}$, which is basically a renaming of attributes in $W$. Formally, for each attribute $A \in W$, we have $\bar{A} \in \bar{W}$ (i.e., $\bar{W}=\{\bar{A}: A \in W\}$ ).

Definition 3 (Views Evolution and Bounded Evolution) Given an instance $\mathbf{r}$ of $R$, an instance $\tau_{J}^{\mathbf{r}}$ of schema $R_{e v}=J W \bar{W}\{V T, \overline{V T}\}$ is defined as follows:

$\tau_{J}^{\mathbf{r}}=\left\{u \mid \exists t, t^{\prime}\left(\begin{array}{c}r(t) \wedge r\left(t^{\prime}\right) \wedge t[J]=t^{\prime}[J]=u[J] \wedge u[W]=t[W] \wedge \\ u\left[\bar{W}=t^{\prime}[W] \wedge t[V T]=u[V T] \wedge t^{\prime}[V T]=u[\overline{V T}]\right. \\ \wedge t[V T]<t^{\prime}[V T] \wedge \\ \forall t^{\prime \prime}\left(\left(r\left(t^{\prime \prime}\right) \wedge t[V T]<t^{\prime \prime}[V T]\right) \rightarrow t^{\prime}[V T] \leq t^{\prime \prime}[V T]\right)\end{array}\right)\right\}$

Schema $R_{e v}$ is called the evolution schema of $R$. We will denote as $\tau_{J}^{R}$ the view Evolution on $R$ that is built by expression $\tau_{J}^{\mathbf{r}}$ for every instance $\mathbf{r}$ of $R$. View $\tau_{J}^{R}$ joins two tuples $t_{1}$ and $t_{2}$ that agree on the values of the attributes in $J$ (i.e. $t_{1}[J]=t_{2}[J]$ ), if $t_{2}$ immediately follows $t_{1}$. More precisely, such tuples are joined if $t_{1}[V T]<t_{2}[V T]$ and there does not exist a tuple $t \in \mathbf{r}$ with $t[J]=t_{1}[J]$ and $t_{1}[V T]<t[V T]<t_{2}[V T]$ (i.e., there does not exist a tuple that holds at some point in between the valid times of such tuples).

For application purposes, it would be important to consider in an evolution schema only those pairs of consecutive tuples whose difference between $\overline{V T}$ and $V T$ is within some given bound. Given a parameter $k \in \mathbb{N} \cup\{+\infty\}$, tuples of $\tau_{J}^{\mathbf{r}}$ are filtered by means of the selection $\Delta_{k}\left(\tau_{J}^{\mathbf{r}}\right)=\sigma_{\overline{V T}-V T \leq k}\left(\tau_{J}^{\mathbf{r}}\right)$ (notice that $\Delta_{+\infty}\left(\tau_{J}^{\mathbf{r}}\right)=\tau_{J}^{\mathbf{r}}$ ).

We will denote as $\Delta_{k}\left(\tau_{J}^{\mathbf{r}}\right)$ the view Bounded Evolution. It forces to consider only those tuples belonging to $\tau_{J}^{\mathbf{r}}$ having a temporal distance within the given threshold $k$. In the following, given a tuple $t \in \tau_{J}^{\mathbf{r}}$, we denote its temporal distance $t[\overline{V T}]-t[V T]$ with $\Delta(t)$.

Let us now define, by using the introduced temporal view Evolution, a slightly restricted version of Pure Temporally Evolving Functional Dependency with respect to that defined in [7]. Without loss of generality, such definition will allow us to simplify the notation and to focus on a general kind of temporal evolution of considered data. 
Definition 4 (Pure Temporally Evolving Functional Dependency) A Pure Temporally Evolving Functional Dependency over the temporal schema $R=U \cup\{V T\}, P E$-FD for short, is an expression of the form:

$\left[\Delta_{k}\left(\tau_{J}^{R}\right)\right] X \bar{Y} \rightarrow \bar{Z}$.

We have that $X \subseteq W$ and $\bar{Y}, \bar{Z} \subseteq \bar{W}$ with $X \neq \emptyset$ and $|Z|=1$ ( $Z$ contains a single attribute). An instance $\mathbf{r}$ of $R$ fulfills a $P E$-FD $\left[\Delta_{k}\left(\tau_{J}^{R}\right)\right] X \bar{Y} \rightarrow \bar{Z}$, written $\mathbf{r} \vDash\left[\Delta_{k}\left(\tau_{J}^{R}\right)\right] X \bar{Y} \rightarrow \bar{Z}$, if and only if for each pair of tuples $t, t^{\prime} \in \Delta_{k}\left(\tau_{J}^{\mathbf{r}}\right)$ we have $\left(t[X]=t^{\prime}[X] \wedge t[\bar{Y}]=t^{\prime}[\bar{Y}]\right) \rightarrow t[\bar{Z}]=t^{\prime}[\bar{Z}]$.

A PE-FD could express dependencies as "A common therapy follows the same symptom for all patients."

Now, we introduce two specializations of $P E$-FDs. Given a $P E$-FD $\left[\Delta_{k}\left(\tau_{J}^{R}\right)\right] X \bar{Y} \rightarrow \bar{Z}$, if set $\bar{Y}=\emptyset$ (i.e., the dependency is $\left.\left[\Delta_{k}\left(\tau_{J}^{R}\right)\right] X \rightarrow \bar{Z}\right)$, we will say that the PE-FD is simple. Moreover, if the considered $P E$-FD is of the type $\left[\Delta_{k}\left(\tau_{J}^{R}\right)\right] X \bar{Y} \rightarrow \bar{X}$, we will say that the $P E$-FD is an update. $P E$-FDs featuring both the properties (i.e., $P E$-FDs of type $\left[\Delta_{k}\left(\tau_{J}^{R}\right)\right] X \rightarrow \bar{X}$ ) are called simple updates. A graphical account of such classes is given in Fig. 2.

\section{Approximate Pure Temporally Evolving Functional Dependencies}

We add approximation to $P E$-FDs in a very similar way we did for FDs in "Background and Related Work" section. First, we specialize the measurement $G_{3}$, which considers the minimum number of tuples in $\mathbf{r}$ to be deleted for the FD to hold, to deal with $P E-F D$ as follows:

$G_{3}\left(\left[\Delta_{k}\left(\tau_{J}^{R}\right)\right] X \bar{Y} \rightarrow \bar{Z}, \mathbf{r}\right)=|\mathbf{r}|-\max \{|\mathbf{s}|:$

$\left.\mathbf{s} \subseteq \mathbf{r}, \mathbf{s} \vDash\left[\Delta_{k}\left(\tau_{J}^{R}\right)\right] X \bar{Y} \rightarrow \bar{Z}\right\}$

By means of $G_{3}$, we can define the relative scaled measurement $g_{3}$ as follows:

$$
\begin{aligned}
& g_{3}\left(\left[\Delta_{k}\left(\tau_{J}^{R}\right)\right] X \bar{Y} \rightarrow \bar{Z}, \mathbf{r}\right) \\
& \quad=\frac{G_{3}\left(\left[\Delta_{k}\left(\tau_{J}^{R}\right)\right] X \bar{Y} \rightarrow \bar{Z}, \mathbf{r}\right)}{|\mathbf{r}|} .
\end{aligned}
$$

Now we are ready to define the Approximate Pure Temporally Evolving Functional Dependency.

Definition 5 (Approximate Pure Temporally Evolving Functional Dependency) An Approximate Pure Temporally Evolving Functional Dependency over the temporal schema $R=U \cup\{V T\}, A P E$-FD for short, is an expression of the form:

$\left[\Delta_{k}\left(\tau_{J}^{R}\right)\right] X \bar{Y} \stackrel{\epsilon}{\rightarrow} \bar{Z}$

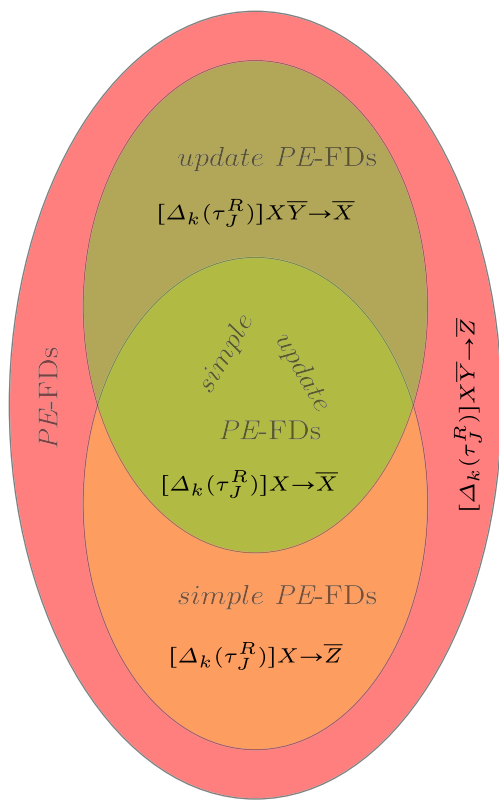

Fig. 2 A graphical account of how different classes of $P E$-FDs are related

with $0 \leq \epsilon \leq 1, X \subseteq W$ and $\bar{Y}, \bar{Z} \subseteq \bar{W}$ with $X \neq \emptyset$ and $|Z|=1$.

An instance $\mathbf{r}$ of $R$ satisfies the APE-FD $\left[\Delta_{k}\left(\tau_{J}^{R}\right)\right] X$ $\bar{Y} \stackrel{\epsilon}{\rightarrow} \bar{Z}$, written $\mathbf{r} \vDash\left[\Delta_{k}\left(\tau_{J}^{R}\right)\right] X \bar{Y} \stackrel{\epsilon}{\rightarrow} \bar{Z}$, if and only if $g_{3}\left(\left[\Delta_{k}\left(\tau_{J}^{R}\right)\right] X \bar{Y} \rightarrow \bar{Z}, \mathbf{r}\right) \leq \epsilon$.

\section{Some Motivating Clinical Scenarios}

In this section, we describe and discuss two scenarios, borrowed from the clinical domain, in order to provide examples of how $P E$-FDs and $A P E$-FDs work. The first scenario is taken from psychiatric case register. Let us consider the temporal schema Contact $=\{$ Name, Phys, $C T, D u r\} \cup\{V T\}$. Such a schema stores values about a phone-call service provided to psychiatric patients. This service is intended for monitoring and helping psychiatric patients, who are not hospitalized. Whenever a patient feels the need to talk to a physician, she can call the service. Data about calls are collected according to schema Contact. For the sake of simplicity, temporal attribute $V T$ identifies the day when the call has been received. In addition, the service may be used by people somehow related to patients, as, for instance, relatives worried about the current condition of a patient.

More precisely, attribute Name identifies patients, Phys identifies physicians, $C T$ (Contact Type) specifies the person who is doing the call (e.g., value "self" stands for the patient himself, "family" for a relative) and Dur stores information about total duration of calls (value $\sim n$ means approximately $n$ minutes). 
Fig. 3 An instance $\mathbf{r}$ of schema Contact that stores the phone contacts about two psychiatric cases. Attribute \# represents the tuple number, and it is used only for referencing tuples in the text (i.e., \# does not belong to the schema Contact)

\begin{tabular}{|c|c|c|c|c|c|}
\hline \# & Name & Phys & $C T$ & Dur(minutes) & $V T$ \\
\hline 1 & McMurphy & Sayer & self & $\sim 15$ & 1 Jan 2016 \\
\hline 2 & McMurphy & Sayer & family & $\sim 5$ & 5 Jan 2016 \\
\hline 3 & McMurphy & Maguire & family & $\sim 15$ & 10 Jan 2016 \\
\hline 4 & McMurphy & Maguire & self & $\sim 15$ & $15 \operatorname{Jan} 2016$ \\
\hline 5 & McMurphy & Maguire & self & $\sim 5$ & $20 \operatorname{Jan} 2016$ \\
\hline$=6$ & McMurphy & Maguire & self & $\sim 5$ & 25 Jan 2016 \\
\hline 7 & Lowe & Sayer & family & $\sim 15$ & 7 Jan 2016 \\
\hline 8 & Lowe & Sayer & family & $\sim 15$ & 15 Jan 2016 \\
\hline 9 & Lowe & Maguire & self & $\sim 15$ & 22 Jan 2016 \\
\hline 10 & Lowe & Sayer & self & $\sim 5$ & 28 Jan 2016 \\
\hline 11 & Lowe & Maguire & self & $\sim 15$ & 3 Feb 2016 \\
\hline 12 & Lowe & Sayer & self & $\sim 5$ & 6 Feb 2016 \\
\hline
\end{tabular}

$\tau_{\text {Name }}^{\mathbf{r}}$

\begin{tabular}{c||c|c|c|c|c|c|c|c|c}
$\#$ & Name & Phys & $C T$ & Dur & $V T$ & $\overline{P h y s}$ & $\overline{C T}$ & $\overline{D u r}$ & $\overline{V T}$ \\
\hline 1,2 & McMurphy & Sayer & self & $\sim 15$ & 1 Jan 2016 & Sayer & family & $\sim 5$ & 5 Jan 2016 \\
2,3 & McMurphy & Sayer & family & $\sim 5$ & 5 Jan 2016 & Maguire & family & $\sim 15$ & 10 Jan 2016 \\
3,4 & McMurphy & Maguire & family & $\sim 15$ & 10 Jan 2016 & Maguire & self & $\sim 15$ & 15 Jan 2016 \\
4,5 & McMurphy & Maguire & self & $\sim 15$ & 15 Jan 2016 & Maguire & self & $\sim 5$ & 20 Jan 2016 \\
5,6 & McMurphy & Maguire & self & $\sim 5$ & 20 Jan 2016 & Maguire & self & $\sim 5$ & 25 Jan 2016 \\
7,8 & Lowe & Sayer & family & $\sim 15$ & 7 Jan 2016 & Sayer & family & $\sim 15$ & 15 Jan 2016 \\
8,9 & Lowe & Sayer & family & $\sim 15$ & 15 Jan 2016 & Maguire & self & $\sim 15$ & 22 Jan 2016 \\
9,10 & Lowe & Maguire & self & $\sim 15$ & 22 Jan 2016 & Sayer & self & $\sim 5$ & 28 Jan 2016 \\
10,11 & Lowe & Sayer & self & $\sim 5$ & 28 Jan 2016 & Maguire & self & $\sim 15$ & 3 Feb 2016 \\
11,12 & Lowe & Maguire & self & $\sim 15$ & 3 Feb 2016 & Sayer & self & $\sim 5$ & 6 Feb 2016 \\
\hline
\end{tabular}

Fig. 4 The evolution expression $\tau_{\text {Name }}^{\mathrm{r}}$

An instance $\mathbf{r}$ of $R$ is provided in Fig. 3. Instance $\tau_{\text {Name }}^{\mathbf{r}}$, and $\tau_{J}^{\mathbf{r}}$ in general, may be seen as the output of a two-phase procedure. First, table Contact is partitioned into subsets of tuples, one for each value of Name. Then, each tuple is joined with its immediate successor in its partition, w.r.t. $V T$ values. The whole relation $\tau_{\text {Name }}^{\mathrm{r}}$ is provided in Fig. 4. In the following, we will use $t$ for referencing tuples of $\mathbf{r}$ and $u$ for referencing tuples of $\tau_{J}^{\mathbf{r}}$. Moreover, in the following, each tuple $u$ in $\tau_{J}^{\mathbf{r}}$ will be identified by the pair of indexes of the tuples in $\mathbf{r}$ that generate $u$. For instance, the first tuple of $\tau_{J}^{\mathbf{r}}$ in Fig. 4 will be denoted by $u_{1,2}$ since it is generated by the join of tuples $t_{1}$ and $t_{2}$ in $\mathbf{r}$.

Going back to our example, it is worth noting that tuples $t_{2}$ and $t_{7}$ are not joined in $\tau_{\text {Name }}^{\mathbf{r}}$, even if $t_{7}[V T]=t_{2}[V T]+2$ and there is no tuple $t$ with $t[V T]=t_{7}[V T]+1$. This is due to the fact that $t_{7}[$ Name $] \neq t_{2}[$ Name $]$ forbids the join in $\tau_{\text {Name }}^{\mathbf{r}}$. Moreover, $t_{1}$ and $t_{3}$ are not joined in $\tau_{\text {Name }}^{\mathbf{r}}$. Indeed, the presence of tuple $t_{2}$ with $t_{1}[$ Name $]=t_{2}[$ Name $]=t_{3}[$ Name $]$ and $t_{1}[V T]<t_{2}[V T]<t_{3}[V T]$ forbids the join in $\tau_{\text {Name }}^{\mathbf{r}}$. Figure 5 graphically depicts how pairs of tuples $\left(t_{1}, t_{2}\right),\left(t_{2}, t_{3}\right),\left(t_{3}, t_{4}\right)$, $\left(t_{4}, t_{5}\right),\left(t_{5}, t_{6}\right)$ and $\left(t_{7}, t_{8}\right),\left(t_{8}, t_{9}\right),\left(t_{9}, t_{10}\right),\left(t_{10}, t_{11}\right),\left(t_{11}, t_{12}\right)$ are joined in $\tau_{\text {Name }}^{\mathbf{r}}$ for the two patients, respectively. Basically, each tuple $u \in \tau_{\text {Name }}^{\mathrm{r}}$ corresponds to an edge in Fig. 5, while we have a node for each tuple in $\mathbf{r}$.

Let us now discuss some temporal dependencies we can derive from such data. We could be interested in verifying whether there is some relationship between some previous features of patient's call and the fact that the considered call was either with him or with a relative. In our example, we have that $\mathbf{r} \vDash\left[\Delta_{5}\left(\tau_{\text {Name }}^{\text {Contact }}\right)\right]$ Phys, $\overline{\text { Phys }} \rightarrow \overline{C T}$.

In other words, given consecutive calls related to the same patient within 5 days, the couple composed by the physician of the first call and by the physician of the next one determines the type of contact of the next call. And it holds for all patients. However, if we consider a wider time window of 6 days, we have that $\mathbf{r} \not \models\left[\Delta_{6}\left(\tau_{\text {Name }}^{\text {Contact }}\right)\right]$ Phys, $\overline{P h y s} \rightarrow \overline{C T}$, because of pairs $\left(t_{2}, t_{3}\right)$ and $\left(t_{10}, t_{11}\right)$. More precisely, we have that $t_{2}[$ Name $]=t_{3}[$ Name $]=$ "McMurphy",$\quad t_{10}[$ Name $]=t_{11}$ $[$ Name $]=$ "Lowe", $\quad t_{2}[$ Phys $]=t_{10}[$ Phys $]=$ "Sleepy", $t_{3}[$ Phys $]=t_{11}[$ Phys $]=$ "Patel", but $t_{3}[C T] \neq t_{11}[C T]$ (i.e., $t_{3}[C T]=$ "family", and $t_{11}[C T]=$ "self"). In other words, we have that the set of tuples $\left\{u_{2,3}, u_{10,11}\right\}$ does not satisfy the FD Phys, $\overline{P h y s} \rightarrow \overline{C T}$. 
Fig. 5 A graph-based representation of $\tau_{\text {Name }}^{\mathbf{r}}$. Nodes represent tuples and are labeled by the corresponding tuple number. Values for attribute Dur are reported above each node. Values of Phys and $C T$ attributes are reported below every node, respectively. Every edge $\left(t_{i}, t_{j}\right)$ is labeled by value $\Delta\left(u_{i, j}\right)=t_{j}[V T]-t_{i}[V T]$ (i.e., the temporal distance between two tuples). The dashed edge represents a different scenario where $t_{2}$ and $t_{4}$ are joined, if $t_{3}$ would be deleted, as explained below for APE-FDs
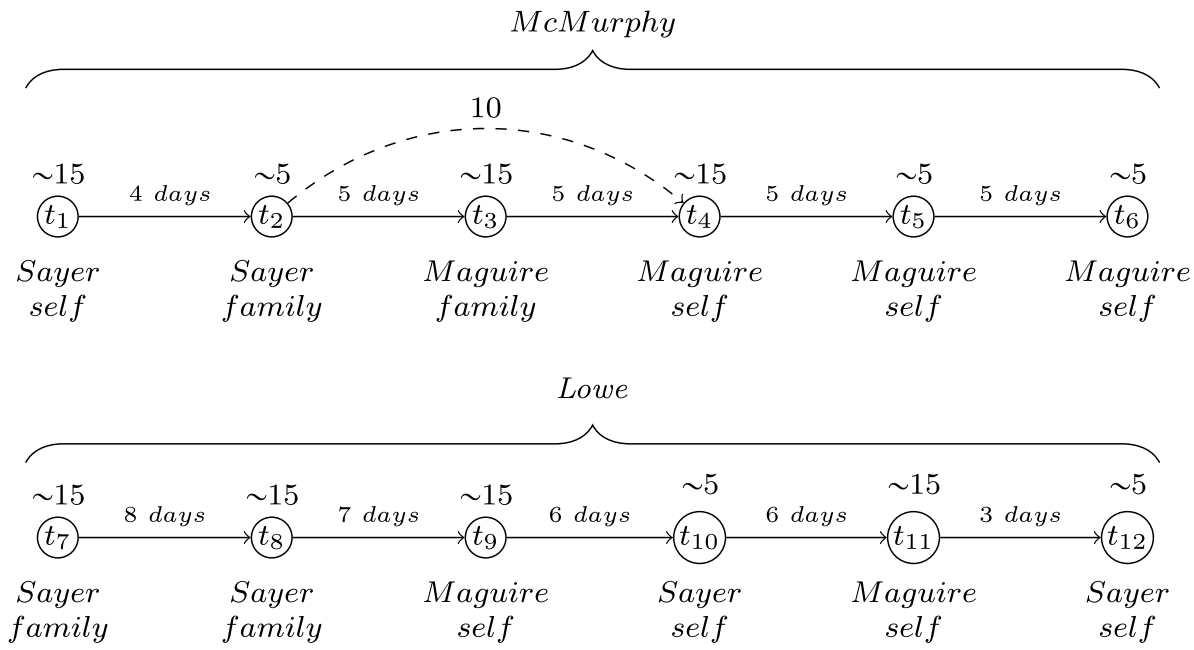

The two proposed PE-FDs differ only for the maximum temporal distance allowed. In particular, tuple $u_{10,11}$ is one of the responsible ones for $\mathbf{r} \not \forall\left[\Delta_{6}\left(\tau_{\text {Name }}^{\text {Contact }}\right)\right]$ Phys, $\overline{\text { Phys }} \rightarrow \overline{C T}$, but it does not belong to $\Delta_{5}\left(\tau_{\text {Name }}^{\text {Contac }}\right)$ because $\Delta\left(u_{10,11}\right)>5$. This allows us to point out a general property of $P E$-FDs and of $A P E$-FDs too. Given a $P E$-FD $\left[\Delta_{k}\left(\tau_{J}^{R}\right)\right] X \bar{Y} \rightarrow \bar{Z}$, if we have that for every instance $\mathbf{r}$ of $R$ it holds $\mathbf{r} \vDash\left[\Delta_{k}\left(\tau_{J}^{R}\right)\right] X \bar{Y} \rightarrow \bar{Z}$, then for every $h \leq k$ it holds $\mathbf{r} \vDash\left[\Delta_{h}\left(\tau_{J}^{R}\right)\right] X \bar{Y} \rightarrow \bar{Z}$.

Moving to the problem of mining approximate dependencies, if we consider APE-FD $\left[\Delta_{6}\left(\tau_{\text {Name }}^{R}\right)\right] P h y s, \overline{P h y s} \leftrightarrow \bar{\epsilon} \rightarrow \overline{C T}$ with $\epsilon=\frac{1}{12}$, we have that $\mathbf{r} \vDash\left[\Delta_{6}\left(\tau_{\text {Name }}^{R}\right)\right]$ Phys, $\overline{P h y s} \stackrel{\epsilon}{\rightarrow} \overline{C T}$. Indeed, by considering relation $\mathbf{r}^{\prime}=\mathbf{r} \backslash\left\{t_{3}\right\}$, this dependency would hold without the need of approximation (i.e., if tuple $t_{3}$ is deleted from relation $\mathbf{r}$ ). More precisely, we have $\tau_{\text {Name }}^{\mathbf{r}^{\prime}}=\tau_{\text {Name }}^{\mathbf{r}} \backslash\left\{u_{2,3}, u_{3,4}\right\} \cup\left\{u_{2,4}\right\}$. Tuple $u_{2,4}$ was not originally in $\tau_{\text {Name }}^{\mathbf{r}}$ because of tuple $t_{3}$. Figure 5 depicts this new scenario, by replacing edges $\left(t_{2}, t_{3}\right)$ and $\left(t_{3}, t_{4}\right)$ with the dashed edge $\left(t_{2}, t_{4}\right)$. Moreover, we have $\mathbf{r}^{\prime} \vDash\left[\Delta_{+\infty}\left(\tau_{\text {Name }}^{R}\right)\right]$ Phys, $\overline{\text { Phys }} \rightarrow \overline{C T}$. Thus, it holds $\mathbf{r} \vDash\left[\Delta_{+\infty}\left(\tau_{\text {Name }}^{R}\right)\right]$ Phys, $\overline{\text { Phys }} \stackrel{\epsilon}{\rightarrow} \overline{C T}$ with $\epsilon=\frac{1}{12}$.

The second example we propose is borrowed from the internal medicine domain. As another simple example of how view $\tau_{J}^{R}$ works, let us consider the temporal schema ThCy $=\{$ PatId, Phys, Dos $\} \cup\{V T\}$. Such a schema allows one to store the values about cycles of therapies in which a specific, fixed, drug is administered to a patient by a given physician. Figure 6 a depicts an instance $\mathbf{r}$ of $R$. Figure $6 \mathrm{~b}$ shows the result of view $\tau_{\text {Patld }}^{\text {ThCy }}$ to $\mathbf{r}$. It is easy to see that tuples $t_{1}$ and $t_{5}$ are not joined in $\tau_{\text {Patd }}^{\mathbf{r}}$. Even if $t_{5}[V T]=t_{1}[V T]+1$ the fact that $t_{1}[$ PatId $] \neq t_{5}[$ PatId $]$ forbids the join in $\tau_{\text {Patld }}^{\mathbf{r}}$. Moreover $t_{1}$ and $t_{3}$ are not joined in $\tau_{\text {Patld }}^{\mathbf{r}}$. Even if $t_{1}[$ PatId $]=t_{3}[$ PatId $]$, we have that the presence of tuple $t_{2}$ with $t_{1}[$ PatId $]=t_{2}[$ PatId $]=t_{3}[$ PatId $]$ and $t_{1}[V T]<t_{2}[V T]<t_{3}[V T]$ forbids the join in $\tau_{\text {Patld }}^{\mathrm{r}}$. In Fig. 7a, we have a graphical account of how the pairs of tuples $\left(t_{1}, t_{2}\right),\left(t_{2}, t_{3}\right),\left(t_{3}, t_{4}\right),\left(t_{5}, t_{6}\right),\left(t_{6}, t_{7}\right)$ and $\left(t_{7}, t_{8}\right)$ are joined in $\tau_{\text {Patld }}^{\mathbf{r}}$. In both the graphs depicted in Fig. 7a, nodes are labeled with the tuple number and the value for Dos attribute is reported above each node. Moreover, we recall from the previous example that each edge $\left(t_{i}, t_{j}\right)$ is labeled with the value $t_{j}[V T]-t_{i}[V T]$ (i.e., the temporal distance between two tuples). In the scenario for $\tau_{\text {Patld }}^{\mathbf{r}}$ the value for the attribute Phys is reported below each node, while for $\tau_{\text {Phys }}^{\mathbf{r}}$ the value of PatId is reported below each node.

We would like to point out that it may be the case that a tuple $t \in \mathbf{r}$ has a more than one immediate successor in $\tau_{J}^{\mathbf{r}}$ (i.e. is joined with more than one tuple). It is the case of view $\tau_{\text {Phys }}^{\mathbf{r}}$ shown in Fig. 7b, where tuples are joined with respect to the values of attribute Phys. We have that, since Dr. Shepherd makes two drug administrations at $V T=20$, tuple $t_{1}$ has both tuples $t_{3}$ and $t_{7}$ as its immediate successors. We will see that the number of immediate successors of a tuple in $\tau_{J}^{\mathbf{r}}$ will play a major role in some of the following complexity results.

In this domain, we could be interested in understanding whether there are dependencies among previous and current drug dosages for a given patient, possibly considering the physicians administering the drug.

In the example depicted in Figs. 6 and 7, we have that $\mathbf{r} \vDash\left[\Delta_{+\infty}\left(\tau_{\text {Patld }}^{R}\right)\right]$ Dos, Phys, $\overline{\text { Phys }} \rightarrow \overline{\text { Dos }}$. It means that the dosage and the couple of physicians related to a drug administration and to the next one, respectively, determine the next drug dosage. However, $\mathbf{r} \backslash \vDash\left[\Delta_{\infty}\left(\tau_{\text {Patld }}^{R}\right)\right]$ Phys, $\overline{\text { Phys }} \rightarrow \overline{\text { Dos }}$ because both tuples $u_{1,2}$ and $u_{7,8}$ belong to $\Delta_{\infty}\left(\tau_{\text {Patld }}^{R}\right)$. More precisely, we have that Phys, $\overline{P h y s} \rightarrow \overline{D o s}$ does not hold on any instance of $\Delta_{k}\left(\tau_{\text {Patld }}^{R}\right)$ that contains both $u_{1,2}$ and $u_{7,8}$, since $u_{1,2}[$ Phys $]=u_{7,8}[$ Phys $]=$ "Shepherd" and $u_{1,2}[\overline{\text { Phys }}]=u_{7,8}[\overline{\text { Phys }}]=$ "Stevens" but $u_{1,2}[\overline{\text { Dos }}]=$ "60 mg" is not equal to $u_{7,8}[\overline{D o s}]=$ "20 $\mathrm{mg}$ ". 
Fig. 6 The instances $\tau_{\text {Patld }}^{\mathbf{r}}(\mathbf{b})$ and $\tau_{P h y s}^{\mathbf{r}}(\mathbf{c})$ obtained from applying views $\tau_{\text {Patld }}^{\text {ThCy }}$ and $\tau_{\text {Phys }}^{\text {ThCy }}$ to the instance $\mathbf{r}(\mathbf{a})$, respectively

\begin{tabular}{cc|c|c|c|c} 
(a) & PatId & Phys & Dos & VT \\
\hline 1 & 1 & Shepherd & $30 \mathrm{mg}$ & 1 May 2016 \\
2 & 1 & Stevens & $60 \mathrm{mg}$ & 5 May 2016 \\
3 & 1 & Shepherd & $40 \mathrm{mg}$ & 20 May 2016 \\
$\mathbf{r}=$ & 1 & Shepherd & $40 \mathrm{mg}$ & 27 May 2016 \\
4 & 2 & Stevens & $30 \mathrm{mg}$ & 2 May 2016 \\
5 & 2 & Stevens & $40 \mathrm{mg}$ & 9 May 2016 \\
6 & 2 & Shepherd & 60 mg & 20 May 2016 \\
8 & 2 & Stevens & 20 mg & 25 May 2016 \\
\hline
\end{tabular}

(b)

\begin{tabular}{cc||c|c|c|c|c|c|c} 
(b) & \#atId & Phys & Dos & VT & $\overline{\text { Phys }}$ & $\overline{\text { Dos }}$ & $\overline{V T}$ \\
\hline 1,2 & 1 & Shepherd & $30 \mathrm{mg}$ & 1 May 2016 & Stevens & $60 \mathrm{mg}$ & 5 May 2016 \\
$\tau_{\text {PatId }}^{\mathbf{r}}=$ & 1 & Stevens & $60 \mathrm{mg}$ & 5 May 2016 & Shepherd & $40 \mathrm{mg}$ & 20 May 2016 \\
& 3,4 & 1 & Shepherd & $40 \mathrm{mg}$ & 20 May 2016 & Shepherd & $40 \mathrm{mg}$ & 27 May 2016 \\
5,6 & 2 & Stevens & $30 \mathrm{mg}$ & 2 May 2016 & Stevens & $40 \mathrm{mg}$ & 9 May 2016 \\
6,7 & 2 & Stevens & $40 \mathrm{mg}$ & 9 May 2016 & Shepherd & $60 \mathrm{mg}$ & 20 May 2016 \\
& 7,8 & 2 & Shepherd & $60 \mathrm{mg}$ & 20 May 2016 & Stevens & 20 mg & 25 May 2016 \\
\hline
\end{tabular}

\begin{tabular}{cc|c|c|c|c|c|c|c}
$(c)$ & $\#$ & Phys & PatId & Dos & $V T$ & $\overline{P a t I d}$ & $\overline{D o s}$ & $\overline{V T}$ \\
\hline 1,3 & Shepherd & 1 & $30 \mathrm{mg}$ & 1 May 2016 & 1 & $40 \mathrm{mg}$ & 20 May 2016 \\
1,7 & Shepherd & 1 & $30 \mathrm{mg}$ & 1 May 2016 & 2 & $60 \mathrm{mg}$ & 20 May 2016 \\
$\tau_{\text {Phys }}^{\mathbf{r}}=3,4$ & Shepherd & 1 & $40 \mathrm{mg}$ & 20 May 2016 & 1 & $40 \mathrm{mg}$ & 27 May 2016 \\
7,4 & Shepherd & 2 & $60 \mathrm{mg}$ & 20 May 2016 & 1 & $40 \mathrm{mg}$ & 27 May 2016 \\
5,2 & Stevens & 2 & $30 \mathrm{mg}$ & 2 May 2016 & 1 & $60 \mathrm{mg}$ & 5 May 2016 \\
2,6 & Stevens & 1 & $60 \mathrm{mg}$ & 5 May 2016 & 2 & $40 \mathrm{mg}$ & 9 May 2016 \\
6,8 & Stevens & 2 & $40 \mathrm{mg}$ & 9 May 2016 & 2 & $20 \mathrm{mg}$ & 25 May 2016 \\
\hline
\end{tabular}

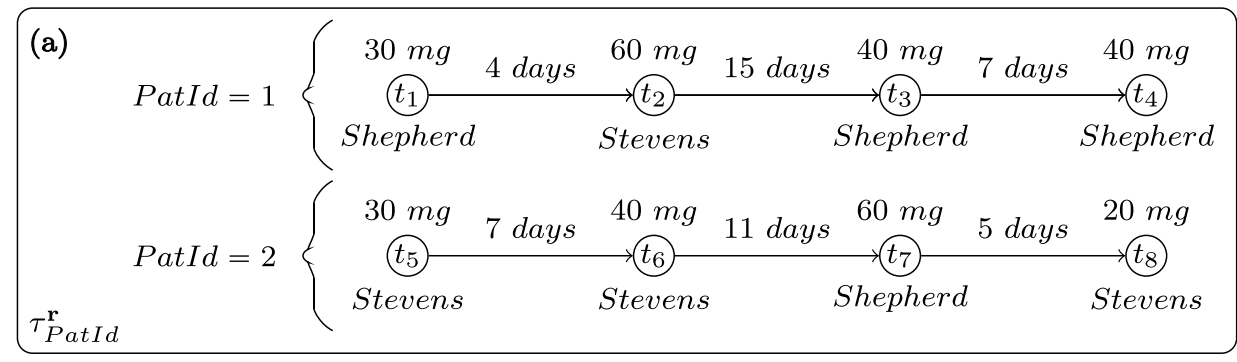

Fig. 7 A graphical account for the instances $\tau_{\text {Patld }}^{\mathbf{r}}(\mathbf{a})$ and $\tau_{\text {Phys }}^{\mathbf{r}}$ (b) related to the instance $\mathbf{r}$ shown in Fig. 6a

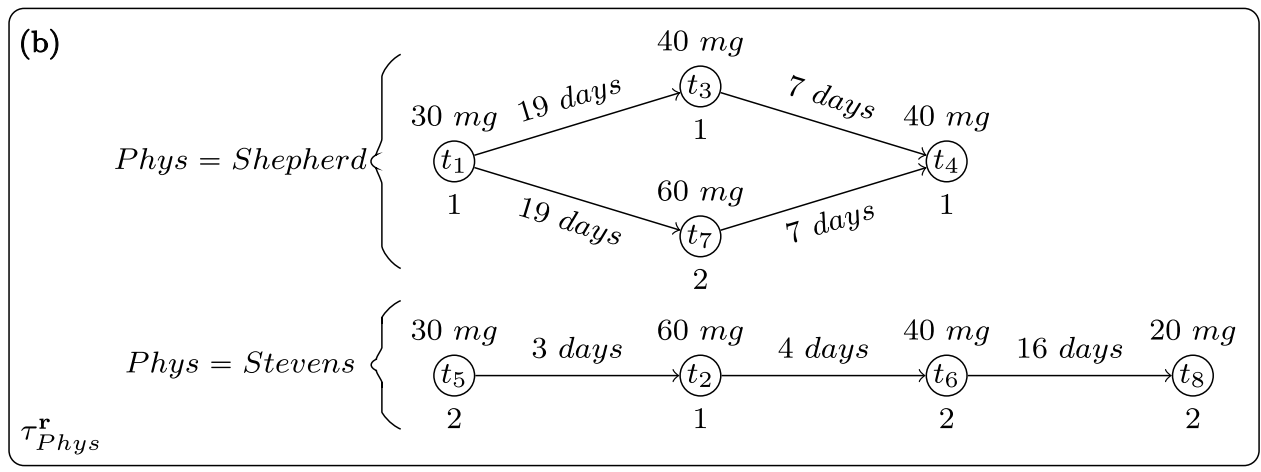

On the other hand, $\mathbf{r} \vDash\left[\Delta_{4}\left(\tau_{\text {Patld }}^{R}\right)\right]$ Phys, $\overline{\text { Phys }} \rightarrow \overline{\text { Dos }}$ because having 4 as maximum allowed time distance implies $u_{7,8} \notin \Delta_{4}\left(\tau_{\text {Patld }}^{\mathbf{r}}\right)$ (i.e., $t_{8}[V T]-t_{7}[V T]>4$ ) and thus the conflict between $u_{1,2}$ and $u_{7,8}$ no longer exists. Furthermore, it is easy to see by considering the pairs $u_{1,2}$ and $u_{5,6}$ that $\mathbf{r} \backslash \vDash\left[\Delta_{+\infty}\left(\tau_{\text {Patld }}^{R}\right)\right] D o s, \overline{\text { Phys }} \rightarrow \overline{\text { Dos }}$. However, by shrinking the maximum allowed time distance to 6 , we obtain $u_{5,6} \notin \Delta_{6}\left(\tau_{P_{a t t d}}^{\mathrm{r}}\right)$ and thus we have that $\mathbf{r} \vDash\left[\Delta_{6}\left(\tau_{\text {Patld }}^{R}\right)\right]$ Dos, $\overline{\text { Phys }} \rightarrow$ Dos.

Obviously, in an instance $\tau_{J}^{\mathbf{r}}$, there may be more than one pair $\left(u, u^{\prime}\right)$ which generates a conflict. Let us consider the simple update $P E-\mathrm{FD}\left[\Delta_{+\infty}\left(\tau_{\text {Patld }}^{R}\right)\right] \operatorname{Dos} \rightarrow \overline{\operatorname{Dos}}$. Such $P E$ FD does not hold on $\mathbf{r}$ (i.e., $\mathbf{r} \backslash \vDash\left[\Delta_{+\infty}\left(\tau_{\text {Patld }}^{R}\right)\right] D o s \rightarrow \overline{D o s}$ ). This can be shown using the pair $u_{1,2}$ and $u_{5,6}$ as a witness for a conflict since $u_{1,2}[D o s]=u_{5,6}[D o s]=" 30 \mathrm{mg}$ " and 
$u_{1,2}[\overline{D o s}] \neq u_{5,6}[\overline{D o s}]$. However, in this case, we have that the conflicting pairs are more than one. As a matter of fact, all pairs $\left(u_{1,2}, u_{5,6}\right),\left(u_{2,3}, u_{7,8}\right)$ and $\left(u_{3,4}, u_{4,6}\right)$ are conflictgenerating. If we want to rule all the conflicts out by playing on the maximum allowed distance, we have to set it to 6 and then we have $\mathbf{r} \vDash\left[\Delta_{6}\left(\tau_{\text {Patld }}^{R}\right)\right]$ Dos $\rightarrow \overline{\text { Dos. }}$.

\section{The Computational Complexity of Checking APE-FD}

In this section, we address the complexity of checking an $A P E-F D$ against an instance $\mathbf{r}$. We call this problem CheckAPE-FD:

Problem 1 (Check- $A P E$-FD). Given a temporal schema $R$, a $P E-\mathrm{FD}\left[\Delta_{k}\left(\tau_{J}^{R}\right)\right] X \bar{Y} \rightarrow \bar{Z}$ on $R$, an instance $\mathbf{r}$ of $R$, and a real number $0 \leq \epsilon \leq 1$, determine whether $\mathbf{r} \vDash\left[\Delta_{k}\left(\tau_{J}^{R}\right)\right] X \bar{Y} \stackrel{\epsilon}{\rightarrow} \bar{Z}$ or not.

Let us consider, for example, the $P E$-FD

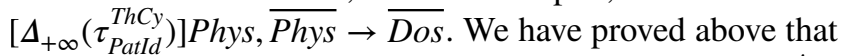
$\mathbf{r} \backslash \vDash f d$. Figure 8 graphically reports all the possible $\tau_{\text {Patld }}^{\mathbf{r}^{\prime}}$ where $\mathbf{r}^{\prime}$ is obtained from $\mathbf{r}$ by deleting exactly one tuple. For example, if $\mathbf{r}^{\prime}=\mathbf{r} \backslash\left\{t_{1}\right\}$, it means that the dotted edge $\left(t_{1}, t_{2}\right)$ has been removed. This means that $t_{1}$ and $t_{2}$ are not joined in $\tau_{\text {Patld }}^{\mathbf{r}^{\prime}}$. Moreover, if we take $\mathbf{r}^{\prime}=\mathbf{r} \backslash\left\{t_{2}\right\}$ we have that both the edges $\left(t_{1}, t_{2}\right)$ and $\left(t_{2}, t_{3}\right)$ are removed and the dashed edge $\left(t_{1}, t_{3}\right)$ turns out to be "active." This means that $t_{1}$ and $t_{2}$ are not joined in $\tau_{\text {Patld }}^{\mathbf{r}^{\prime}}$ as well as $t_{2}$ and $t_{3}$, but $t_{1}$ and $t_{3}$ turn out to be joined in $\tau_{\text {Patld }}^{\mathbf{r}^{\prime}}$ due to the absence of $t_{2}$. Let us observe that in this case, the join operation involving $t_{1}$ and $t_{3}$ belongs to $\tau_{\text {Patld }}^{\mathbf{r}^{\prime}}$ and not to $\tau_{\text {Patld }}^{\mathbf{r}}$. This specific behavior, in which the deletion of a tuple introduces additional, possibly different, constraints as a side effect, instead of just removing existing ones, gives us a hint on the problem Check- APE-FD. Such problem is not so easy to solve. Notice that $\mathbf{r} \backslash\left\{t_{1}\right\} \backslash \vDash\left[\Delta_{+\infty}\left(\tau_{\text {Patld }}^{\text {ThCy }}\right)\right]$ Phys, $\overline{\text { Phys }} \rightarrow \overline{\text { Dos }}$ as well, because of the pairs $\left(t_{2}, t_{3}\right)$ and $\left(t_{6}, t_{7}\right)$. However, $\mathbf{r} \backslash\left\{t_{2}\right\} \vDash\left[\Delta_{+\infty}\left(\tau_{P \text { tId }}^{\text {ThCy }}\right)\right]$ Phys, $\overline{\text { Phys }} \rightarrow \overline{\text { Dos }}$ and thus we have that $\mathbf{r} \vDash\left[\Delta_{+\infty}\left(\tau_{\text {Patld }}^{\text {Thy }}\right)\right]$ Phys, $\overline{\text { Phys } \rightarrow} \rightarrow \overline{\text { Dos }}$ with $\epsilon=\frac{1}{8}$.

Therefore, problem Check-APE-FD belongs to the complexity class $N P$. In order to prove that, it suffices to apply a guess-and-check algorithm. First, this algorithm guesses a set $\mathbf{r}^{\prime}$ with $\left|\mathbf{r}^{\prime}\right| \leq \epsilon \cdot|\mathbf{r}|$. Then, if $\mathbf{r} \backslash \mathbf{r}^{\prime} \vDash\left[\Delta_{k}\left(\tau_{J}^{R}\right)\right] X \bar{Y} \rightarrow \bar{Z}$, the algorithm returns $Y E S$, otherwise $N O$. In the procedure above, we implicitly make use of a function that verifies, given an instance $\mathbf{r}$ of $R$ and a $P E-\mathrm{FD}\left[\Delta_{k}\left(\tau_{J}^{R}\right)\right] X \bar{Y} \rightarrow \bar{Z}$, whether $\mathbf{r} \vDash\left[\Delta_{k}\left(\tau_{J}^{R}\right)\right] X \bar{Y} \rightarrow \bar{Z}$ holds or not. We can call this problem Check-PE-FD. Since there is no approximation,

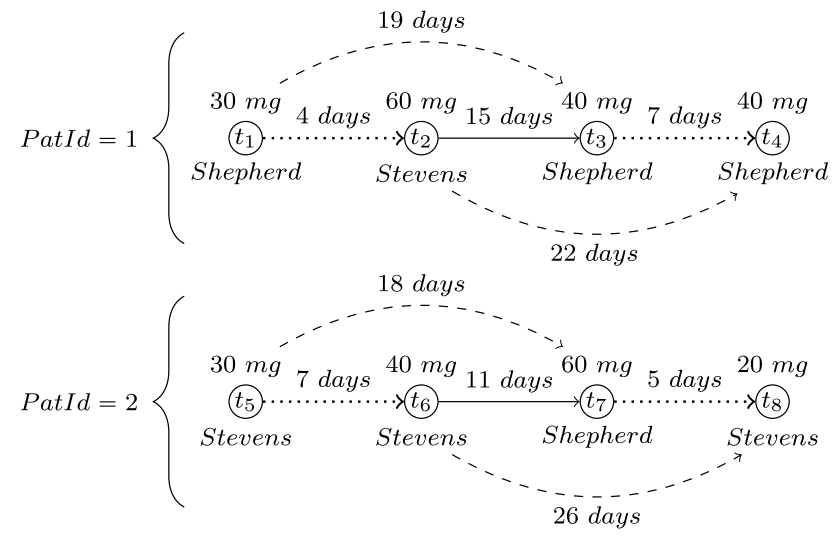

Fig. 8 A graphical account for the possible changes on the view $\tau_{\text {Patld }}^{\mathbf{r}}$ considering the possible deletions of at most one tuple

checking if $\mathbf{r} \vDash\left[\Delta_{k}\left(\tau_{J}^{R}\right)\right] X \bar{Y} \rightarrow \bar{Z}$ may be performed in polynomial time [7]. For this reason, we can conclude that Check$A P E$-FD belongs to the complexity class NP. In the following, we will prove that Check- $A P E$-FD is NP-hard even in the case of the most constrained kind of $A P E$-FDs, which is represented by the class of simple update $A P E$-FDs. From now on, we will consider Problem 1 only for simple update APE-FDs. Considering the inclusions shown in Fig. 2, we can immediately conclude that our hardness result directly propagates to the other classes of APE-FDs.

In this section, we will make use of finite words $w$ on a finite nonempty alphabet $\Sigma$ (i.e., $w \in \Sigma^{*}$ ). We will use the standard notation $w[i]$ for denoting the $i$ th symbol of word $w$. Given a word $w$, we denote with first (w) and last $(w)$ its first and its last element, respectively (i.e., first $(w)=w[1]$ and $\operatorname{last}(w)=w[|w|])$. Moreover, a finite increasing sequence of $\mathbb{N}\left(\mathbb{N}^{>}\right.$-sequence) is a finite word $s$ on $(\mathbb{N} \backslash\{0\})^{*}$, where for every $i, i^{\prime}$, with $1 \leq i<i^{\prime} \leq|s|$, we have $s[i]<s\left[i^{\prime}\right]{ }^{1}$ Given a $\mathbb{N}^{>}$-sequence $s$ we denote with $\operatorname{first}(s)$ and last $(s)$ its first and its last element, respectively (i.e., first $(s)=s[1]$ and $\operatorname{last}(s)=s[|s|])$. A $\mathbb{N}^{>}$-sequence $s$, for which for every $i$, with $1 \leq i<|s|$, we have $w[i+1]=w[i]+1$, is called strict and we denote it with $[b, e]$, where $b=f i r s t(s)$ and $e=$ last $(s)$. Given a word $w$ and a $\mathbb{N}^{>}$-sequence $s$, we denote with $w \|_{s}$ the word $w \|_{s}=w[\operatorname{first}(s)] \ldots w[\operatorname{last}(s)]$ (for a graphical account of how a word is filtered by a sequence please refer to Fig. 9). Given a word $w$ and a pair $(b, e)$, with $1 \leq b \leq e \leq|w|$, we call the word $w \|_{[b, e]}$ a slice of $w$. Given two words $w_{1}, w_{2} \in \Sigma^{*}$ we say that

\footnotetext{
$\overline{1}$ a $\mathbb{N}^{>}$-sequence is nothing more than a different representation for a finite set of positive naturals, but it turns out for our purposes to see it as a particular kind of word over positive naturals.
} 
Fig. 9 An example of a word $w \|_{s}$ obtained by applying a sequence $s$ to a word $w$

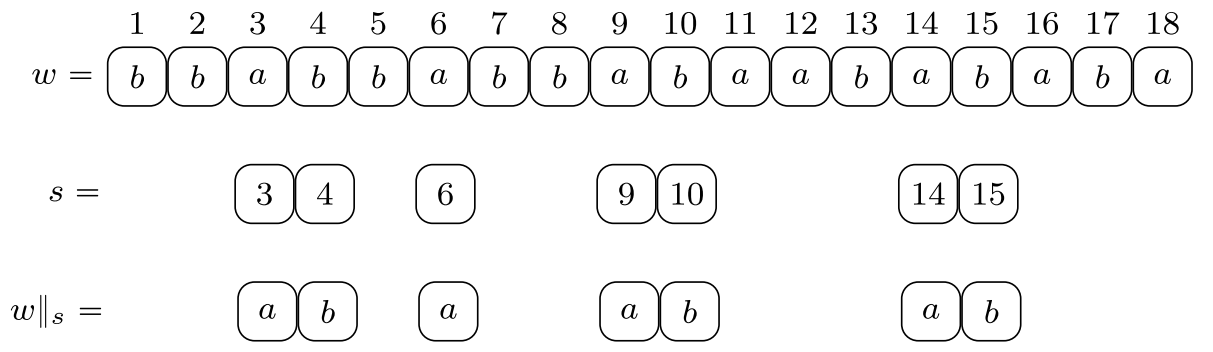

Fig. 10 A graphical account of how $s_{n}^{1}, s_{w_{1}}, s_{n}^{2}, s_{w_{2}}$, and $s_{n}^{3}$ filter blocks of $w$

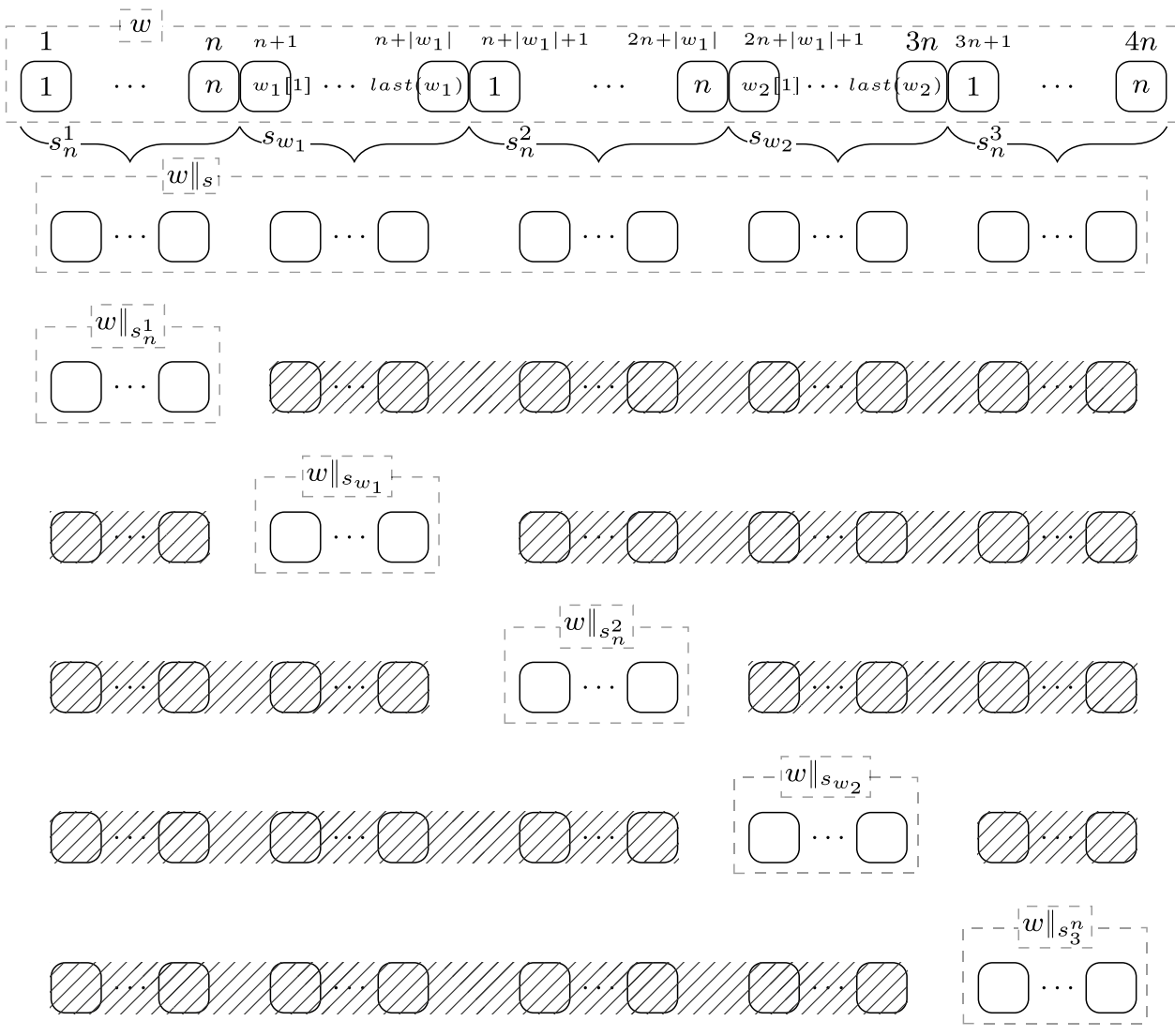

$w_{1}$ is a subsequence of $w_{2}$ (written $w_{1} \sqsubseteq w_{2}$ ) if and only if there exists an $\mathbb{N}^{>}$-sequence $s$ for which $w_{1}=w_{2} \|_{s}$. For instance, $w_{1}=a b a a b b a$ is a subsequence of $w_{2}=$ bbabbabbabaabababa with $s=3469101415$. A word $w$ is repetition free if and only if for every $a \in \Sigma$ we have $|\{i: w[i]=a\}| \leq 1$. A word $w$ is a permutation of $\Sigma$ if and only if $w$ is repetition free and $|w|=|\Sigma|$.

The proof that Check-APE-FD is NP-hard is done in two steps. First, we describe a known NP-Complete problem called Common Permutation Problem ( $C P-\mathrm{P}$ for short). Then, we introduce a problem called Periodic Repair Problem ( $P R-\mathrm{P})$ and we prove that $C P-\mathrm{P}$ may be reduced to it using logarithmic space. Finally, we reduce the $P R-\mathrm{P}$ to Check- $A P E$-FD using logarithmic space.

Let us begin with the Common Permutation Problem which has been proved to be NP-Complete in [12].
Problem 2 (CP-P). Given a finite alphabet $\Sigma$ and two words $w_{1}, w_{2}$ over it, is there a permutation $w_{p}$ of $\Sigma$ for which $w_{p} \sqsubseteq w_{1}$ and $w_{p} \sqsubseteq w_{2}$ ?

Consider $\Sigma=\{a, b, c\}$ we have that the pair $w_{1}=b c b a b$ and $w_{2}=a c c a a c b$ is a positive instance of Problem 2 because $c a b \sqsubseteq w_{1}$ and $c a b \sqsubseteq w_{2}$. On the other hand, the pair $w_{1}=b c b a c$ and $w_{2}=a c a b$ is a negative instance of Problem 2 since both words do not share any permutation of $\Sigma$ as their subsequence. More precisely, $w_{1}$ contains the permutations $b c a, b a c$ and $c b a$ while $w_{2}$ contains the permutations $a c b$ and $c a b$.

A word $w$ is periodic if and only if for every pair of indexes $\left(i, i^{\prime}\right)$, with $1 \leq i, i^{\prime}<|w|$, we have that $w[i]=w\left[i^{\prime}\right]$ implies $w[i+1]=w\left[i^{\prime}+1\right]$. Let us observe that if $w$ is repetition-free, then it is periodic. Moreover, if $w$ is periodic for 
every pair $(b, e)$, with $1 \leq b \leq e \leq|w|$, we have that $w \|_{[b, e]}$ is periodic (i.e., every slice of a periodic word is itself periodic). The following lemma turns out to be useful for our reduction.

Lemma 1 Given a periodic word $w$, if $w$ is not repetition-free, then there exists an index $i<|w|$ such that $\operatorname{last}(w)=w[i]$.

Proof Since $w$ is not repetition free, there exists two indexes $i, i^{\prime}$, with $1 \leq i<i^{\prime} \leq|w|$, such that $w[i]=w\left[i^{\prime}\right]$. We prove the claim by induction on $\Delta=|w|-i^{\prime}$. For the base of the induction, we have $\Delta=0$ and thus the claim trivially holds since $i$ is the index we were looking for. Let us consider $\Delta=n+1$. Since $w$ is periodic and $w[i]=w\left[i^{\prime}\right]$, we have that $w[i+1]=w\left[i^{\prime}+1\right]$. Thus, positions $i+1$ and $i^{\prime}+1$ witness a repetition and since $|w|-\left(i^{\prime}+1\right)<|w|-i^{\prime}=\Delta$ we can apply the inductive hypothesis and prove our claim.

In order to prove that Check- APE -FD is NP-Complete even for simple update $P E$-FD $\left[\Delta_{k}\left(\tau_{J}^{R}\right)\right] X \rightarrow \bar{X}$, we introduce the following intermediate problem called Periodic Repair Problem (PR-P for short):

Problem $3\left(P R\right.$-P) Given a word $w=a_{1} \ldots a_{n}$, a finite alphabet $\Sigma$ and a natural number $k$, determine whether a periodic word $w^{\prime} \sqsubseteq w$ exists such that $\left|w^{\prime}\right| \geq k$.

Problem 3 belongs to the complexity class NP. A simple nondeterministic algorithm for $P R$-P guesses an $\mathbb{N}^{>}$ -sequence $s$ such that $|s| \geq k$ and $\operatorname{last}(s) \leq|w|$ (i.e., $s$ "chooses" only positions in $1 \ldots|w|)$. Then, it suffices to check whether or not $w \|_{s}$ is periodic (periodicity checking may be performed in logarithmic space).

In the following, we describe how to reduce $C P$-P to $P R$ -P. Let us consider two words $w_{1}$ and $w_{2}$ on an alphabet $\Sigma$ with length $n_{1}$ and $n_{2}$, respectively. We assume without loss of generality that $\Sigma$ is a finite subset of the negative integers (i.e., $\left.\Sigma \subseteq \mathbb{Z}^{-}\right)$. Let $n=\max \left(n_{1}, n_{2}\right)$ and $\sigma=|\Sigma|$. Let us consider the following word $w$ over the alphabet $\Sigma \cup\{1, \ldots, n\}$ $(\cdot$ is the classical word concatenation operator):

$w=1 \cdot \ldots \cdot n \cdot w_{1} \cdot 1 \cdot \ldots \cdot n \cdot w_{2} \cdot 1 \cdot \ldots \cdot n$.

Finally, we put $k=3 n+2 \sigma$. Such reduction operates in logarithmic space. The following two lemmas prove the soundness and completeness of the above reduction.

Lemma 2 If there exists a permutation $w_{p}$ of $\Sigma$ which is a common subsequence of $w_{1}, w_{2}$, then there exists $a \mathbb{N}^{>}$-sequence s, with $|s| \geq 3 n+2 \sigma$ and last $(s) \leq 3 n+\left|w_{1}\right|+\left|w_{2}\right|$, such that $w \|_{s}$ is periodic.
Proof First, let us recall that $w_{p}$ is a repetition-free sequence of symbols in $\mathbb{Z}^{-}$. By hypothesis, we have $w_{p} \sqsubseteq w_{1}$ and $w_{p} \sqsubseteq w_{2}$ and thus there exists a pair of $\mathbb{N}^{>}$-sequence $s_{1}$ and $s_{2}$ with $\left|s_{1}\right|=\sigma,\left|s_{2}\right|=\sigma$ and $w_{1}\left\|_{s_{1}}=w_{2}\right\|_{s_{2}}=w_{p}$.

Let $\bar{s}_{j}$ with $j \in\{1,2\}$ be the $\mathbb{N}^{>}$-sequence such that $\left|\bar{s}_{j}\right|=\sigma$ and for every $1 \leq i \leq \sigma$, we have $\bar{s}_{j}[i]=s_{j}[i]+n j+\sigma(j-1)$. Let us observe that $\bar{s}_{j}$ is a simple shift of the indexes in the sequence $s_{j}$ with $j \in\{1,2\}$. Then, we may define $s$ as follows:

$s=\begin{aligned} & {[1, n] \cdot \bar{s}_{1} \cdot\left[n+\left|w_{1}\right|+1,2 n+\left|w_{1}\right|\right] \cdot \bar{s}_{2}} \\ & \cdot\left[2 n+\left|w_{1}\right|+\left|w_{2}\right|+1,3 n+\left|w_{2}\right|+\left|w_{1}\right|\right]\end{aligned}$

By construction, we have $w \|_{s}=1 \ldots n \cdot w_{p} \cdot 1 \ldots n \cdot w_{p} \cdot 1 \ldots n$. Since $\Sigma \cap\{1, \ldots n\}=\emptyset$ there are not "conflicts" between the blocks $1 \ldots n$ and $w_{p}$, we can conclude that $w \|_{s}$ is periodic.

Lemma 3 If there exists a sequence $s$ with $3 n+2 \sigma \leq|s| \leq 3 n+\left|w_{1}\right|+\left|w_{2}\right|$ for which $w \|_{s}$ is periodic, then there exists a permutation $w_{p}$ of $\Sigma$ which is a common subsequence of $w_{1}, w_{2}$.

Proof First, we define $s_{n}^{1}, s_{w_{1}}, s_{n}^{2}, s_{w_{2}}, s_{n}^{3}$ such that $s=s_{n}^{1} \cdot s_{w_{1}}$ $\cdot s_{n}^{2} \cdot s_{w_{2}} \cdot s_{n}^{3}$ and $\operatorname{last}\left(s_{n}^{1}\right) \leq n, \quad n+1 \leq s_{w_{1}}[1] \leq \operatorname{last}\left(s_{w_{1}}\right)$ $\leq n+\left|w_{1}\right|, \quad n+\left|w_{1}\right|+1 \leq s_{n}^{2}[1] \leq \operatorname{last}\left(s_{n}^{2}\right) \leq 2 n+\left|w_{1}\right|$, $2 n+\left|w_{1}\right|+1 \leq s_{w_{2}}[1] \leq \operatorname{last}\left(s_{w_{2}}\right) \leq 2 n+\left|w_{1}\right|+\left|w_{2}\right|$ and $n+\left|w_{1}\right|+\left|w_{2}\right|+1 \leq s_{n}^{3}[1]$. Informally $s_{n}^{1}, s_{w_{1}}, s_{n}^{2}, s_{w_{2}}, s_{n}^{3}$ are the indexes in $s$ that concern the subwords $1 \ldots n$ (first block), $w_{1}, 1 \ldots n$ (second block), $w_{2}$ and $1 \ldots n$ (third block) respectively. A graphical account of this decomposition of $w \|_{s}$ is given in Fig. 10. This means that we may retrieve the subsequence selected by $s$ on $w$ restricted to the first block by means of the operation $w \|_{s_{n}^{1}}$. If we want to retrieve the subsequence selected by $s$ on $w$ restricted to the $w_{1}$ block, we write $w \|_{s_{w_{1}}}$, and so on, for the second block $1 \ldots n$ (i.e., $w \|_{s_{n}^{2}}$, the block $w_{2}$ (i.e., $w \|_{s_{w_{2}}}$ ) and the third block $1 \ldots n$ (i.e., $w \|_{s_{n}^{3}}$ ). Let us notice that $w \|_{s}$ is equal to $w\left\|_{s_{n}^{1}} \cdot w\right\|_{s_{w_{1}}}$. $w\left\|_{S_{n}^{2}} \cdot w\right\|_{S_{w_{2}}} \cdot w \|_{S_{n}^{3}}$.

Suppose by contradiction that $\left|s_{n}^{2}\right|=0$. Then, we have that $w^{\prime}=w\left\|_{s_{w_{1}}} \cdot w\right\|_{s_{w_{2}}}$ is a slice of $w \|_{s}$ and thus $w^{\prime}$ is periodic. Moreover, we have that $w^{\prime \prime}=w^{\prime} \cdot\left(w \|_{s_{n}^{3}}\right)$ is a slice of $w \|_{s}$ and thus $w^{\prime \prime}$ is periodic. Two cases may arise, i.e., either $\left|s_{n}^{3}\right|=0$ or not.

If $\left|s_{n}^{3}\right|=0$ we have that $w \|_{s} \sqsubseteq 1 \ldots n \cdot w^{\prime}$ and by a counting argument we have that $\left|w \|_{s}\right| \leq 3 n$ which is a contradiction since $3 n+2 \sigma \leq\left|w \|_{s}\right|$ and $\sigma>0$ by definition.

If $\left|s_{n}^{3}\right|>0$, we prove that $w^{\prime \prime}$ is repetition free. Again by contradiction, from Lemma 1, we will have that, since $w^{\prime \prime}$ is periodic, there must exist an index $i<\left|w^{\prime \prime}\right|$ for which $w^{\prime \prime}[i]=w^{\prime \prime}[n]$. However, $\left|s_{n}^{3}\right|>0$ implies $w^{\prime \prime}[n] \in\{1, \ldots, n\}$ and thus, since $\Sigma \cap\{1, \ldots, n\}=\emptyset$, such $i^{\prime}$ cannot exist 
(contradiction). If $w^{\prime \prime}$ is repetition free, we have that $\left|w^{\prime \prime}\right| \leq \sigma+n$, and since $w \|_{s} \sqsubseteq 1 \ldots n \cdot w^{\prime \prime}$, we have that $\left|w \|_{s}\right| \leq 2 n+\sigma$, which contradicts $\left|w \|_{s}\right| \geq 3 n+2 \sigma$.

We have now that $\left|s_{n}^{2}\right|>0$. Consider the slice $w^{\prime}=w\left\|_{s_{w_{1}}} \cdot w\right\|_{s_{n}^{2}}$ : we have just proved that $\left|w \|_{s_{n}^{2}}\right|>0$ and we have that $w^{\prime}$ is periodic being a slice of $w \|_{s}$. By applying Lemma 1 as we did above, we can claim that $w \|_{s_{w_{1}}}$ is repetition free and thus $\left|w \|_{s_{w_{1}}}\right| \leq \sigma$. Suppose now by contradiction that $w \|_{s_{w_{2}}}$ is not repetition free. If $\left|s_{n}^{3}\right|>0$ we reach immediately a contradiction by applying Lemma 1 on the word $w\left\|_{s_{w_{2}}} \cdot w\right\|_{s_{n}^{3}}$. Then, we have $\left|s_{n}^{3}\right|=0$ and by definition $\left|w \|_{s_{w_{2}}}\right| \leq n$. This implies $w\left\|_{s} \sqsubseteq 1 \ldots n \cdot w\right\|_{s_{w_{1}}} \cdot 1 \ldots n \cdot w \|_{s_{w_{2}}}$ which means $\left|w \|_{s}\right| \leq 3 n+\sigma$ (contradiction).

At this point, we have that both $w \|_{s_{w_{1}}}$ and $w \|_{s_{w_{2}}}$ are repetition free and thus $\left|w \|_{s_{w_{1}}}\right| \leq \sigma$ and $\left|w \|_{s_{w_{2}}}\right| \leq \sigma$. Since $3 n+2 \sigma \leq\left|w \|_{s}\right|$, we have that $\left|w \|_{s_{w_{1}}}\right|=\sigma$ and $\left|w \|_{s_{w_{2}}}\right|=\sigma$ and thus both $w \|_{s_{w_{1}}}$ and $w \|_{s_{w_{2}}}$ are permutations of $\Sigma$. It remains to prove that they are the same permutation. Let us observe that, since $\left|w\left\|_{s_{w_{1}}}|=| w\right\|_{s_{w_{2}}}\right|=\sigma$ and $\left|w \|_{s}\right| \geq 3 n+2 \sigma$, by a counting argument we have that $w\left\|_{S_{n}^{1}}=w\right\|_{s_{n}^{2}}=w \|_{s_{n}^{3}}=1 \ldots n$. and thus $w\left\|_{s}=1 \ldots n \cdot w\right\|_{s_{w_{1}}} \cdot 1 \ldots n \cdot w \|_{s_{n}^{2}} \cdot 1 \ldots n$.

Suppose by contradiction that there exists $i$, with $1 \leq i \leq \sigma$, such that $w\left\|_{S_{w_{1}}}[i] \neq w\right\|_{S_{w_{2}}}[i]$, and let $i$ be the minimum index that fulfills such a property. Two cases may arise:

1. If $i=1$ we have that $w\left\|_{s}[n+1]=w\right\|_{s_{w_{1}}}[i]$ and $w\left\|_{s}[2 n+\sigma+1]=w\right\|_{s_{w_{2}}}[i]$. Let us recall that $w\left\|_{s}[n]=w\right\|_{s}[2 n+\sigma]=n$, and thus by periodicity of $w \|_{s}$, we have $w\left\|_{s}[n+1]=w\right\|_{s}[2 n+\sigma+1]$ (contradiction).

2. If $i>1$ since $w \|_{s}$ is periodic, we have that $w\left\|_{s_{w_{1}}}[i-1] \neq w\right\|_{s_{w_{2}}}[i-1]$ but this contradicts the minimality in the choice of $i$.

Now we reduce Problem $P R$-P to Check-APE-FD in logarithmic space. Suppose that we have an instance of $P R$-P consisting of a word $w \in \Sigma^{*}$ and a natural number $k$. We define the instance $\mathbf{r}_{w}$ on the temporal schema $R=\{J, X\} \cup V T$ as $\mathbf{r}_{w}=\{t \mid t[J]=0 \wedge \exists i(t[X]=w[i] \wedge t[V T]=i)\}$, and we put $\epsilon_{w, k}=\frac{|w|-k}{|w|}$. The pair $w, k$ is a positive instance of $P R$-P if and only if the triple $\left[\Delta_{+\infty}\left(\tau_{J}^{R}\right)\right] X \rightarrow \bar{X}, \mathbf{r}_{w}$ and $\epsilon_{w, k}$ is a positive instance of Check-APE-FD. $\left[\Delta_{+\infty}\left(\tau_{J}^{R}\right)\right] X \rightarrow \bar{X}, \mathbf{r}_{w}$ and $\epsilon_{w, k}$ may be built using logarithmic space on the input $w, k$. Finally, we can conclude this section by explicitly providing the desired result.

Theorem 1 Problem Check-APE-FD is NP-Complete.

\section{Algorithms for Checking APE-FDs}

As we proved in "The Computational Complexity of Checking APE-FD" section, given an APE-FD $\left[\Delta_{k}\left(\tau_{J}^{R}\right)\right] X \bar{Y} \stackrel{\epsilon}{\rightarrow} \bar{Z}$ and an instance $\mathbf{r}$ of $R$, the problem Check- $A P E$-FD is NP-Complete in $|\mathbf{r}|$. Then, in principle, there is no asymptotically better algorithm than exploring the whole set of possible subsets $\mathbf{r}^{\prime}$ of $\mathbf{r}$ with $\frac{|\mathbf{r}|-\left|\mathbf{r}^{\prime}\right|}{|\mathbf{r}|} \leq \epsilon$.

In the following, we provide two algorithms that make use of heuristics, for pruning the search space in order to achieve the tractability for many cases.

The first algorithm is the more general one, and it may be applied without assumptions on the input instance $\mathbf{r}$. Such algorithm makes use of two optimization techniques. The first one consists of trying, whenever it is possible, to split the current subset of $\mathbf{r}$ into two subsets, on which the problem may be solved independently (i.e., choices in one subset do not affect those in the other one and vice versa). The latter optimization technique consists of checking whether the current partial solution may not lead to an optimal solution (i.e., a solution $\mathbf{r}^{\prime}$ where $\left|\mathbf{r}^{\prime}\right|$ is the maximum possible number of tuples that may be kept). If this happens, the subtree is pruned immediately (i.e., we are looking only for optimal solutions).

The second algorithm is applicable under the assumption that we have a bounded and relatively small number of tuples that share the same values for both $V T$ and $J$ which is often the case in clinical domains, as we will discuss later on. In this setting, we show how to provide an upper bound value for all the candidate solutions that contain the current partial solution and thus we can apply a pure branch-and-bound approach in order to speed up the algorithm even more.

Before discussing in detail the algorithms and their properties, we need to introduce some basic concepts and features for the representation of tuples through graph-based structures.

\section{Graph-Based Structures for Tuple Representation}

To this regard, we use a suitable graph representation of tuples. A directed graph is a pair $G=(V, E)$, where $V$ is a finite set of nodes and $E \subseteq\left(\begin{array}{l}V \\ 2\end{array}\right)$ is its edge set. Our graphs are simple, i.e., there are no loops and no parallel edges. Let $U \subseteq V$ : we denote by $\left.G\right|_{U}=\left(U,\left.E\right|_{U}\right)$ the subgraph of $G$ induced by $U$, that is, the graph on node set $U$ such that, for every $u, v \in U,(u, v)$ is an edge in $\left.G\right|_{U}$ if and only if $(u, v)$ is an edge in $G$. A Layered Directed Acyclic Graph ( $L-D A G$ for short) is a triple $\mathcal{L}_{G}=(V, E, l)$ where $(V, E)$ is a DAG and $l$ is a function $l: V \rightarrow\{1, \ldots, p\}$ for some $p \in \mathbb{N}$ where for every $(u, v) \in E$ we have $l(u)<l(v)$. We define the jump value of an edge $(u, v) \in E$ as jump $(u, v)=l(v)-l(u)$. For each $i \in\{1, \ldots, p\}$, we denote 


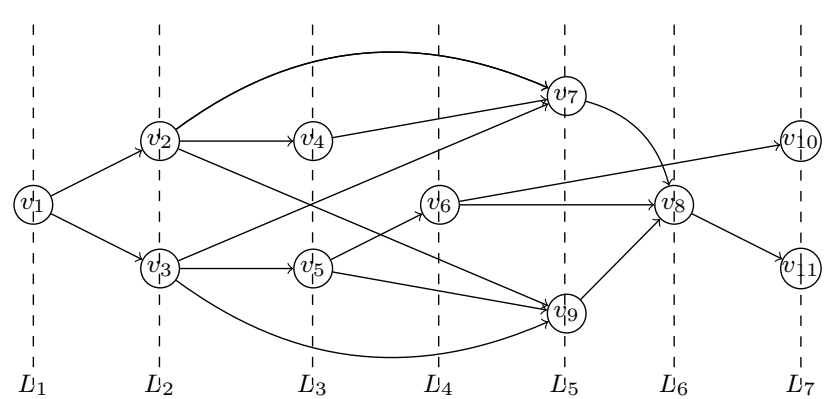

Fig. 11 An example of $L-D A G$

with $L_{i}$ the set $L_{i}=\{v \in V: l(v)=i\}$. Obviously, the notion of induced subgraph naturally extends to $L-D A G$. Given an $L-D A G \mathcal{L}_{G}=(V, E, l)$ and a subset $U$, we define the $L-D A G$ induced by $U$ as $\left.\mathcal{L}_{G}\right|_{U}=\left(U,\left.E\right|_{U},\left.l\right|_{U}\right)$ where $\left(U,\left.E\right|_{U}\right)$ is the $U$-induced subgraph of the graph $(V, E)$ and $\left.l\right|_{U}: U \rightarrow \mathbb{N}$ with $\left.l\right|_{U}(v)=l(v)$ for every $v \in U$. An example of $L-D A G \mathcal{L}_{G}=(V, E, l)$ is given in Fig. 11. Edges $\left(v_{2}, v_{7}\right),\left(v_{2}, v_{9}\right),\left(v_{3}, v_{7}\right)$ and $\left(v_{3}, v_{9}\right)$ have jump value equal to 3. For instance, for $\mathcal{L}_{G}$, we may change the layering function $l$ into $l^{\prime}$ where $L_{i}=L_{i}^{\prime}$ for every $i=1, \ldots 4, L_{5}^{\prime}=L_{5} \backslash\left\{v_{7}\right\}$ and $L_{6}^{\prime}=L_{6} \cup\left\{v_{7}\right\}$.

We extend the notion of $L-D A G$ with weights, denoting it as $w L-D A G$. A $w L-D A G$ is expressed through the tuple $\mathcal{W L}_{G}=(V, E, l, \mathcal{W})$, where $\mathcal{L}_{G}=(V, E, l)$ is an $L-D A G$ and $\mathcal{W}$ is a function $\mathcal{W}: V \rightarrow \mathbb{N}^{+}$. Let us notice that in our notion of weighted $L-D A G$, weights are associated with nodes. Let us now introduce a general problem on $L-D A G$, called $k$-Thick Path ( $k$-TP for short).

Problem $4(k-T P)$. Given an $L-D A G \mathcal{L}_{G}=(V, E, l)$ and a natural number $k$, determine whether or not there exists a node subset $V^{\prime} \subseteq V$, such that $\left|V^{\prime}\right| \geq k$ and for every $u, v \in V^{\prime}$, with $l(u)<l(v)$, there exists a directed path from $u$ to $v$ in $\left.\mathcal{L}_{G}\right|_{V^{\prime}}$.

For instance, if we consider the $L-D A G$ in Fig. 11, we have that the set $V^{\prime}=\left\{v_{1}, v_{2}, v_{3}, v_{7}, v_{9}, v_{8}, v_{11}\right\}$ is a possible solution for $k$-TP with $k \leq 7$ while $V^{\prime \prime}=\left\{v_{1}, v_{2}, v_{3}, v_{4}, v_{7}, v_{8}, v_{11}\right\}$ is not a candidate solution since there is no path from $v_{3}$ to $v_{4}$ and $l\left(v_{3}\right)<l\left(v_{4}\right)$. In a solution, we may choose to take more than one node per layer as well as completely ignore all the nodes in a layer. Then, we may see a candidate solution $V^{\prime}$ as the result of a two-step nondeterministic guess:

1. First we select a set of $p^{\prime} \leq p$ layers $\left\{l_{1}, \ldots l_{p^{\prime}}\right\} \subseteq\{1, \ldots, p\}$ (let us assume $l_{i}<l_{j}$ for every $1 \leq i<j \leq p^{\prime}$ ), which will be all and only the layers which contain at least one node in our solution;
2. For $1 \leq i \leq p^{\prime}$ we select a nonempty set $V_{i} \subseteq V$ such that $l(v)=l_{i}$ for every $v \in V_{i}$ and for every $\left(v^{\prime}, v\right) \in V_{i-1} \times V_{i}$, we have $\left(v^{\prime}, v\right) \in E$.

Going back to the example in Fig. 11, in $V^{\prime \prime}$ condition 2 is violated because by choosing $v_{4}$ we choose layer 3 as the nonempty layer following layer 2 but $\left(v_{3}, v_{4}\right) \notin E$. As a matter of fact, $V^{\prime \prime} \backslash\left\{v_{3}\right\}$ (i.e., we choose only $v_{2}$ in the layer 2) turns out to be candidate solution. In $V^{\prime}$, we ignore layers 3 and 4 by not choosing any node in them. Instead, we choose layer 5 as the nonempty layer following layer 2 and everything works just fine.

The $k$-TP problem may naturally be extended to $w L$ $D A G$ by imposing the set $V^{\prime}$ to satisfy $\Sigma_{v \in V^{\prime}} \mathcal{W}(v) \geq k$. In [10], we prove that the $k$-TP problem on $w L-D A G$ is NPhard. Our proof can be naturally extended to prove that the nonweighted version of the problem is NP-hard too.

\section{The First Algorithm}

Both algorithms rely on the concept of color that we will explain through an example in the following. Given an $A P E-$ $\mathrm{FD}\left[\Delta_{k}\left(\tau_{J}^{R}\right)\right] X \bar{Y} \stackrel{\epsilon}{\rightarrow} \bar{Z}$ and an instance $\mathbf{r}$ of $R$, let us suppose that we are solving the problem Check- $A P E$-FD on such instance with a simple guess-and-check procedure, which makes use of two, initially empty, subsets $\mathbf{r}^{+}$(the tuples to be kept in the solution) and $\mathbf{r}^{-}$(the tuples to be deleted in the solution) of $\mathbf{r}$. At each step, the procedure guesses a tuple $t$ in $\mathbf{r} \backslash\left(\mathbf{r}^{+} \cup \mathbf{r}^{-}\right)$ and decides nondeterministically (guessing phase) either to update $\mathbf{r}^{+}$to $\mathbf{r}^{+} \cup\{t\}$ (i.e., $t$ is kept in the current partial solution) or to update $\mathbf{r}^{-}$to $\mathbf{r}^{-} \cup\{t\}$ (i.e., $t$ is deleted in the current partial solution). When $\mathbf{r}=\mathbf{r}^{+} \cup \mathbf{r}^{-}$(checking phase), the procedure returns $Y E S$ if $\mathbf{r}^{+} \vDash\left[\Delta_{k}\left(\tau_{J}^{R}\right)\right] X \bar{Y} \stackrel{\epsilon}{\rightarrow} \bar{Z}$ and $\left|\mathbf{r}^{-}\right| \leq \epsilon \cdot|\mathbf{r}|$, otherwise it returns $N O$. Hereinafter, we call partial solution a triple $\left(\mathbf{r}, \mathbf{r}^{+}, \mathbf{r}^{-}\right)$, such that $\left(\mathbf{r}^{+} \cup \mathbf{r}^{-}\right) \subseteq \mathbf{r}$ and $\mathbf{r}^{+} \cap \mathbf{r}^{-}=\emptyset$. If $\mathbf{r}=\mathbf{r}^{+} \cup \mathbf{r}^{-}$we simply say that $\left(\mathbf{r}, \mathbf{r}^{+}, \mathbf{r}^{-}\right)$ is a solution. A solution $\left(\mathbf{r}, \mathbf{r}^{+}, \mathbf{r}^{-}\right)$is consistent if and only if $\mathbf{r}^{+} \vDash\left[\Delta_{k}\left(\tau_{J}^{R}\right)\right] X \bar{Y} \stackrel{\epsilon}{\rightarrow} \bar{Z}$. Given two partial solutions $\left(\mathbf{r}, \mathbf{r}_{1}^{+}, \mathbf{r}_{1}^{-}\right)$ and $\left(\mathbf{r}, \mathbf{r}_{2}^{+}, \mathbf{r}_{2}^{-}\right)$, we say that $\left(\mathbf{r}, \mathbf{r}_{2}^{+}, \mathbf{r}_{2}^{-}\right)$extends $\left(\mathbf{r}, \mathbf{r}_{1}^{+}, \mathbf{r}_{1}^{-}\right)$if and only if $\mathbf{r}_{1}^{+} \subseteq \mathbf{r}_{2}^{+}$and $\mathbf{r}_{1}^{-} \subseteq \mathbf{r}_{2}^{-}$.

Is there a way to check whether we are generating an inconsistent solution, possibly without guessing all tuples in $\mathbf{r}$ ? Violations of the latter constraint (i.e., $\left|\mathbf{r}^{-}\right| \leq \epsilon \cdot|\mathbf{r}|$ ) are fairly simple to detect during the guessing phase. Indeed, it suffices to check after each insertion in $\mathbf{r}^{-}$if $\left|\mathbf{r}^{-}\right|$exceeds $\epsilon \cdot|\mathbf{r}|$. If it is the case, the procedure may return $N O$ immediately without guessing any further. Violations of the first constraint (i.e., $\mathbf{r}^{+} \vDash\left[\Delta_{k}\left(\tau_{J}^{R}\right)\right] X \bar{Y} \rightarrow \bar{Z}$ ) during the guessing phase are trickier to detect.

When two tuples $t, t^{\prime}$ share the same value for attribute $J$ (i.e., $t[J]=t^{\prime}[J]$ ), we say that they are in the same $J$-group and $t[J]$ is the value of the $J$-group containing $t$ and $t^{\prime}$. For the sake of brevity, for a given $j \in \operatorname{Dom}(J)$ we will use 
$j$-group for denoting the $J$-group with value $j$. An ordered pair, written o-pair, is a pair $\left(t, t^{\prime}\right) \in \mathbf{r} \times \mathbf{r}$ such that $t$ and $t^{\prime}$ are in the same $J$-group and $t[V T]<t^{\prime}[V T]$. We say that a pair $\left(t, t^{\prime}\right)$ is an edge if and only if $0<t^{\prime}[V T]-t[V T] \leq k$. Given a triple $\left(\mathbf{r}, \mathbf{r}^{+}, \mathbf{r}^{-}\right)$, an o-pair $\left(t, t^{\prime}\right)$ is active if and only if $t, t^{\prime} \in \mathbf{r}^{+}$and for every tuple $\bar{t}$ in the same $J$-group of $t$ if $t[V T]<\bar{t}[V T]<t^{\prime}[V T]$, we have $\bar{t} \in \mathbf{r}^{-}$(i.e., $t$ and $t^{\prime}$ are selected in the current partial solution and all the tuples between $t$ and $t^{\prime}$ in the same $J$-group have been deleted). Given two valid times $v t, v t^{\prime} \in \operatorname{Dom}(V T)$ and a value $j \in \operatorname{Dom}(J)$, $v t$ and $v t^{\prime}$ are consecutive in the $j$-group if and only if there exists an active o-pair $\left(t, t^{\prime}\right)$ with $t[V T]=v t, t^{\prime}[V T]=v t^{\prime}$ and $t[J]=j$. It is important to observe that we may have two distinct values $j, j^{\prime} \in \operatorname{Dom}(J)$ and two distinct valid times $v t, v t^{\prime} \in \operatorname{Dom}(V T)$ which are consecutive in $j$-group and not consecutive in $j^{\prime}$-group. Moreover, we may have edges $\left(t, t^{\prime}\right)$ that are not active and active pairs $\left(t, t^{\prime}\right)$ that are not edges; such is the case of active pairs $\left(t, t^{\prime}\right)$ with $t^{\prime}[V T]-t[V T]>k$. A color is a tuple $c$ on the schema $C=X Y Z$, and we say that two colors $(x, y, z)$ and $\left(x^{\prime}, y^{\prime}, z^{\prime}\right)$ are conflicting if and only if $x=x^{\prime}, y=y^{\prime}$, and $z \neq z^{\prime}$. Given an o-pair $\left(t, t^{\prime}\right)$, its color, denoted by $c\left(t, t^{\prime}\right)$, is the tuple $c\left(t, t^{\prime}\right)=\left(t[X], t^{\prime}[Y], t^{\prime}[Z]\right)$. Two o-pairs $\left(t, t^{\prime}\right),\left(t^{\prime \prime}, t^{\prime \prime \prime}\right)$ are conflicting if and only if $c\left(t, t^{\prime}\right)$ and $c\left(t^{\prime \prime}, t^{\prime \prime \prime}\right)$ are conflicting.

Theorem 2 Given an APE-FD $\left[\Delta_{k}\left(\tau_{J}^{R}\right)\right] X \bar{Y} \stackrel{\epsilon}{\rightarrow} \bar{Z}$, an instance $\mathbf{r}$ of $R$, and a partial solution $\left(\mathbf{r}, \mathbf{r}^{+}, \mathbf{r}^{-}\right)$, if there exist two active and conflicting edges $\left(t, t^{\prime}\right)$ and $\left(t^{\prime \prime}, t^{\prime \prime \prime}\right)$, then for every solution $\left(\mathbf{r}, \mathbf{r}_{f}^{+}, \mathbf{r}_{f}^{-}\right)$that extends $\left(\mathbf{r}, \mathbf{r}^{+}, \mathbf{r}^{-}\right)$it holds $\mathbf{r}_{f}^{+} \not \forall\left[\Delta_{k}\left(\tau_{J}^{R}\right)\right] X \bar{Y} \rightarrow \bar{Z}$ (i.e., the solution is inconsistent).

The above theorem guarantees that from a partial solution $\left(\mathbf{r}, \mathbf{r}^{+}, \mathbf{r}^{-}\right)$that features at least two conflicting edges, we cannot reach a consistent solution $\left(\mathbf{r}, \mathbf{r}_{f}^{+}, \mathbf{r}_{f}^{-}\right)$. In such a case, we may return immediately $N O$ without considering any further $\left(\mathbf{r}, \mathbf{r}^{+}, \mathbf{r}^{-}\right)$. The colors of a partial solution $\left(\mathbf{r}, \mathbf{r}^{+}, \mathbf{r}^{-}\right)$are represented by the set $\operatorname{colors}\left(\mathbf{r}, \mathbf{r}^{+}, \mathbf{r}^{-}\right)=\left\{\left(t[X], t^{\prime}[Y], t^{\prime}[Z]\right)\right.$ : $\left(t, t^{\prime}\right)$ is an active edge in $\left.\left(\mathbf{r}, \mathbf{r}^{+}, \mathbf{r}^{-}\right)\right\}$. Clearly, the hypothesis of Theorem 2 applies if and only if set $\operatorname{colors}\left(\mathbf{r}, \mathbf{r}^{+}, \mathbf{r}^{-}\right)$contains at least two conflicting colors.

Then, by means of colors, our above guess-and-check procedure may be improved by adding the control on the size of $\mathbf{r}^{-}$and by keeping updated the current set of colors $\operatorname{colors}\left(\mathbf{r}, \mathbf{r}^{+}, \mathbf{r}^{-}\right)$. Once an insertion of a tuple in either $\mathbf{r}^{+}$or $\mathbf{r}^{-}$introduces a color $c$ that is conflicting with at least one color in $\left(\mathbf{r}, \mathbf{r}^{+}, \mathbf{r}^{-}\right)$, the procedure answers $N O$ immediately. An example of how the procedure works is given in Fig. 12, where we have an instance of five tuples with $\epsilon=0.2$ (i.e., we may delete at most one tuple) and $k=6$ (all the tuples are in the same window). The execution depicted in Fig. 12 guesses the values of tuples from the oldest $\left(t_{1}\right)$ to the newest one $\left(t_{2}\right)$ according to the value of $V T$. First, it tries to put

SN Computer Science a SPRINGER Nature journal. the current tuple $t$ in $\mathbf{r}^{+}$; if no violation arises, it continues; if some violation arises, it tries to insert tuple $t$ in $\mathbf{r}^{-}$; if no violation arises, it continues; otherwise, it goes back to the previous choice (i.e., backtracking). Every internal node is labeled with the current tuple, which will be guessed next; every leaf is labeled either with YES (i.e., the current branch is a solution) or $N O$ (i.e., a violation has arisen); the current set of colors is reported within the node. Nodes are numbered according to their order of appearance. We have that the root is $n_{1}$ followed by the introduction of nodes $n_{2} \ldots n_{4}$ in this precise order. If we introduce $t_{4}$ in the partial solution associated to $n_{4}$, we violate the first constraint. Since in $n_{4}$ adding $t_{4}$ in $\mathbf{r}^{-}$does not generate any violation, node $n_{5}$ is created as child of $n_{4}$. However, node $n_{5}$ cannot be extended without introducing a violation in the above constraints. Indeed, if we put $t_{5}$ in $\mathbf{r}^{+}$, we introduce a conflicting color; if we put $t_{5}$ in $\mathbf{r}^{-}$, we exceed the maximum number of allowed deletions. We backtrack to $n_{4}$. As all the possible choices have been explored, we backtrack to $n_{3}$, where the choice of adding $t_{3}$ to $\mathbf{r}^{-}$is attempted, generating node $n_{6}$. From $n_{6}$, we put $t_{5}$ in $\mathbf{r}^{+}$without violating any constraint and thus we have that $\left\{t_{1}, t_{2}, t_{4}, t_{5}\right\} \vDash\left[\Delta_{6}\left(\tau_{J}^{R}\right)\right] X \bar{Y} \stackrel{0.2}{\longrightarrow} \bar{Z}$.

Let us now consider in some more detail the first algorithm. Basically, the algorithm works similarly to the previous procedure, except for some trivial technicalities. Two more heuristics have been introduced, to possibly stop earlier, during the exploration of a branch in the tree of computation. The main procedure of the algorithm is reported in Fig. 15, while auxiliary procedures are reported in Figs. 13 and 14. The algorithm is implemented by function TupleWiseMin that takes four arguments. The first argument is $G_{\mathbf{r}}$, which is derived from $\mathbf{r}$ considering the $A P E-\mathrm{FD}\left[\Delta_{k}\left(\tau_{J}^{R}\right)\right] X \bar{Y} \stackrel{\epsilon}{\rightarrow} \bar{Z}$ that has to be checked. More precisely, $G_{\mathbf{r}}$ is an instance of schema $J, X, Y, Z, V T$, count, with Dom $($ count $)=\mathbb{N}$. We have that $t \in G_{\mathbf{r}}$ if and only if there exists $t^{\prime} \in \mathbf{r}$ for which $\left(t^{\prime}[J], t^{\prime}[X], t^{\prime}[Y], t^{\prime}[Z]\right)=(t[J], t[X], t[Y], t[Z])$ and $t[$ count $]=\left|\left\{t^{\prime} \in \mathbf{r}:\left(t^{\prime}[J], t^{\prime}[X], t^{\prime}[Y], t^{\prime}[Z]\right)=(t[J], t[X], t[Y], t[Z])\right\}\right|$ that is, we count how many tuples in $\mathbf{r}$ share the same values for attributes $J, X, Y$ and $Z$, respectively. The input parameter $k$ is the length for the grouping sliding window. Sets $G_{\mathbf{r}}^{+}$and $G_{\mathbf{r}}^{-}$, originally initialized to $\emptyset$, represent the tuples of $G_{\mathbf{r}}$ that are either kept or deleted in the current solution, respectively. On instances $s$ of schema $J, X, Y, Z, V T$, count, we denote with $\|r\|$ the sum on the count attribute for the tuples in $s$ (i.e., $\|s\|=\sum_{t \in s} t[$ count $]$ ). Finally, $\mathcal{C}$ is a set of colors which is initially set to $\emptyset$. A color $c$ is a tuple on the schema $X, Y, Z$. As we will see, $\mathcal{C}$ keeps track via colors of the constraints introduced so far in the construction of the solution.

Procedure TupleWiseMin returns the minimum number of tuples that has to be deleted from $\mathbf{r}$ in order to obtain an instance $\mathbf{r}^{\prime}$ such that $\mathbf{r}^{\prime} \vDash\left[\Delta_{k}\left(\tau_{J}^{R}\right)\right] X \bar{Y} \rightarrow \bar{Z}$. Then, if such minimum is less or equal than $\epsilon \cdot|\mathbf{r}|$ we can conclude $\mathbf{r} \vDash\left[\Delta_{k}\left(\tau_{J}^{R}\right)\right] X \bar{Y} \stackrel{\epsilon}{\rightarrow} \bar{Z}$, else we have $\mathbf{r} \backslash \vDash\left[\Delta_{k}\left(\tau_{J}^{R}\right)\right] X \bar{Y} \stackrel{\epsilon}{\rightarrow} \bar{Z}$. 


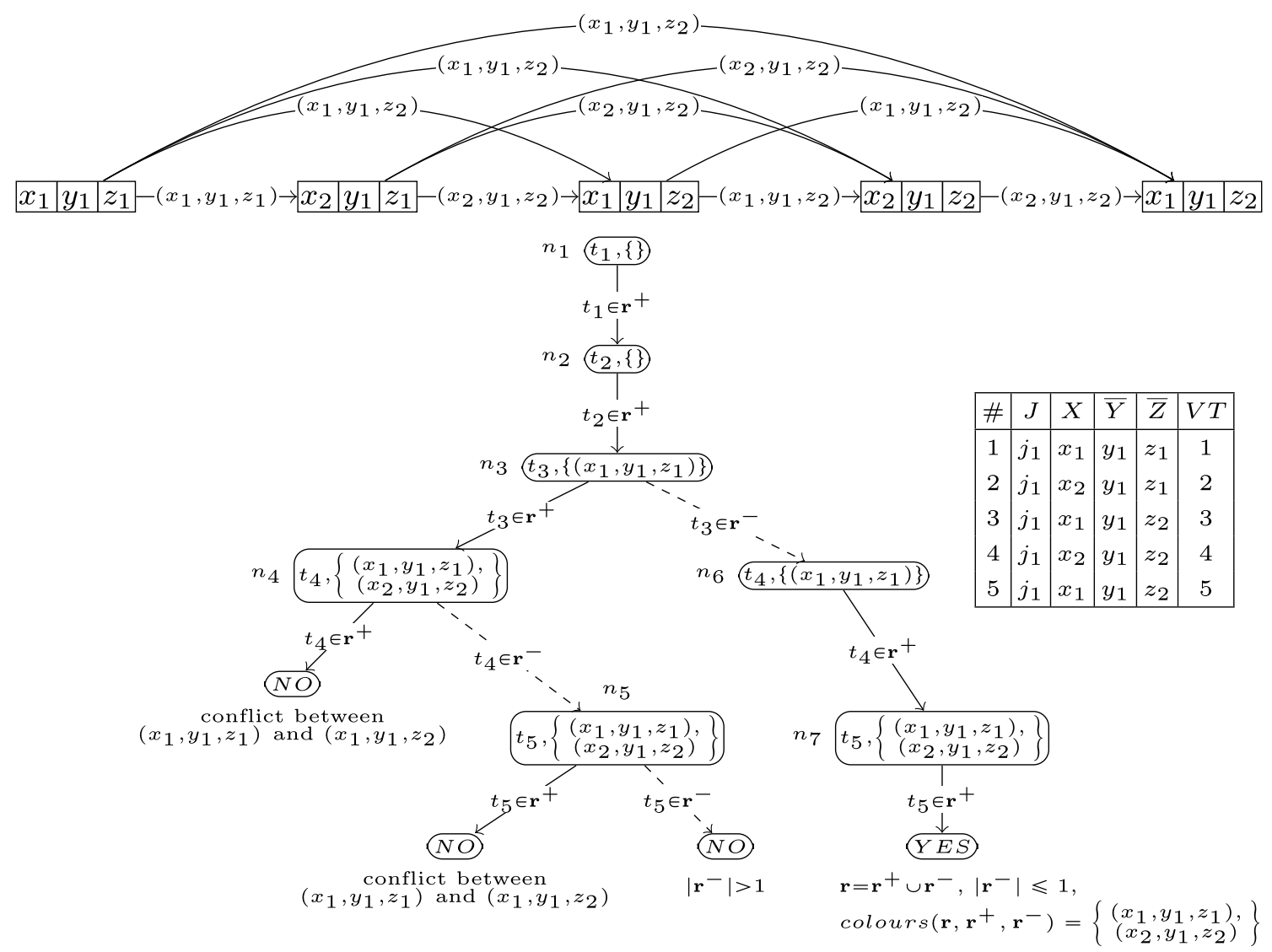

Fig. 12 An example of how the use of colors improves a guess and check procedure for solving the problem Check- $A P E-F D$

Given $G_{\mathbf{r}}, G_{\mathbf{r}}^{+}, G_{\mathrm{r}}^{-}$and a set of colors $\mathcal{C}$ we say that an edge $\left(t, t^{\prime}\right) \in G_{\mathbf{r}} \times G_{\mathbf{r}}$ is pending if and only if the following conditions hold:

1. $t, t^{\prime} \notin G_{\mathbf{r}}^{-}$and $\left(t[X], t^{\prime}[Y], z\right) \notin \mathcal{C}$ for every $z \in \operatorname{Dom}(Z)$;

2. for every $t^{\prime \prime}$ with $t^{\prime \prime}[J]=t[J]$ and $t[V T]<t^{\prime \prime}[V T]<t^{\prime}[V T]$ we have $t^{\prime \prime} \notin G_{\mathbf{r}}^{+}$;

3. there exists $t^{\prime \prime} \in\left(G_{\mathbf{r}} \cup\left\{t, t^{\prime}\right\}\right) \backslash\left(G_{\mathbf{r}}^{-} \cup G_{\mathbf{r}}^{+}\right)$with $t^{\prime \prime}[J]=t[J]$ and $t[V T] \leq t^{\prime \prime}[V T] \leq t^{\prime}[V T]$.

Informally speaking, a pending edge is an edge that is not active in the current partial solution but it may become active during the computation and, if it happens, it introduces a new color in $\mathcal{C}$. In our algorithm, pending edges for the current partial solution are retrieved by procedure $E$ ?, while active edges are retrieved by procedure $E$ !.

Procedure TupleWiseMin (Fig. 15) works as follows. If $G_{\mathbf{r}}^{+} \cup G_{\mathbf{r}}^{-}=G_{\mathbf{r}}$, it means that we have obtained a solution without violating any constraint and thus we can return $\left\|G_{\mathbf{r}}^{-}\right\|$ (i.e., the number of deleted tuples). If $G_{\mathbf{r}}^{+} \cup G_{\mathbf{r}}^{-} \neq G_{\mathbf{r}}$, the algorithm guesses a tuple $t \in G_{\mathbf{r}} \backslash\left(G_{\mathbf{r}}^{+} \cup G_{\mathbf{r}}^{-}\right)$and proceeds as follows. First, it checks whether inserting $t$ into $G_{\mathbf{r}}^{+}$does not cause any violation of constraints. If so, it stores in $m_{t}$ the value of the recursive call to TupleWiseMin where $t$ belongs to $G_{\mathbf{r}}^{+}$and $\mathcal{C}$ has been updated accordingly. By inserting a tuple $t$ in $G_{\mathrm{r}}^{+}$, the algorithm is asserting that $t$ belongs to the current partial solution, while by inserting $t$ in $G_{\mathbf{r}}^{-}$the algorithm is asserting that $t$ does not belong to the current partial solution. If a constraint is violated, the algorithm stores in $m_{t}$ the value $+\infty$, which means that $t$ may not be kept in the current solution.

Then, it checks whether inserting $t$ into $G_{\mathbf{r}}^{-}$does not cause any violation in the constraints. If it is the case, it stores in $m_{\backslash t}$ the value of the recursive call to TupleWiseMin, where $G_{\mathbf{r}}^{-}$and $\mathcal{C}$ are updated accordingly. If a constraint is violated, the algorithm stores in $m_{\backslash t}$ the value $+\infty$, which means that $t$ must be kept in the current partial solution. In procedure TupleWiseMin, the only way in which a constraint may be violated is that, after the insertion a tuple $t$ in $G_{\mathbf{r}}^{+}\left(\operatorname{resp} . G_{\mathbf{r}}^{-}\right.$), an edge $\left(t^{\prime}, t^{\prime \prime}\right)$ turns out to be active and its color $\left(t^{\prime}[X], t^{\prime \prime}[Y], t^{\prime \prime}[Z]\right)$ turns out to be conflicting with at least one color in $\mathcal{C}$.

As pointed out by the example in Fig. 12, checking each step for consistency is itself an optimization, even if it is trivial, since it allows us to prune entire subtrees in the tree of computations without exploring them. We propose 
Fig. 13 Auxiliary procedures used by procedures presented in Figs. 14, 15 and 17

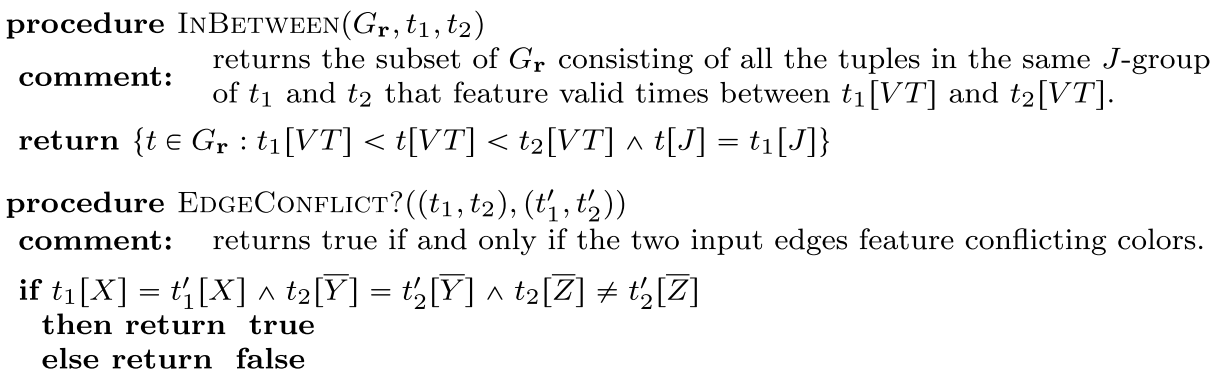

here two further optimizations for this procedure. The first one allows us to restrict the search space by splitting the problem into independent subproblems in a divide-andconquer fashion. Let us suppose that at a certain step of our computation, there exists a value $j \in\left\{t[J]: t \in G_{\mathbf{r}}\right\}$, for which for each pair of conflicting pending edges $\left(t, t^{\prime}\right)$ and $\left(\bar{t}, \bar{t}^{\prime}\right)$ we have that either all $t, t^{\prime}, \bar{t}$, and $\bar{t}^{\prime}$ belong to the $j$-group or all $t, t^{\prime}, \bar{t}$ and $\bar{t}^{\prime}$ do not belong to the $j$-group (such condition is verified by subprocedure Group Independent? reported in Fig. 14.) Let $\bar{G}_{\mathbf{r}}=\{t: t[J]=j\}$. As every edge involving tuples in the $j$-group is not conflicting with every edge that may be introduced outside the $j$-group, then we can split the problem into the two subproblems $\left(\bar{G}_{\mathbf{r}}, k, G_{\mathbf{r}}^{+} \cap \bar{G}_{\mathbf{r}}, G_{\mathbf{r}}^{-} \cap \bar{G}_{\mathbf{r}}\right)$ and $\left(G_{\mathbf{r}} \backslash \bar{G}_{\mathbf{r}}, k, G_{\mathbf{r}}^{+} \backslash \bar{G}_{\mathbf{r}}, G_{\mathbf{r}}^{-} \backslash \bar{G}_{\mathbf{r}}\right)$. Such problems are independent and may be solved separately. The resulting value for the solution is the sum of the values returned by TupleWiseMin applied to both the two subproblems. Let $H=\left|G_{\mathbf{r}} \backslash\left(G_{\mathbf{r}}^{+} \cup G_{\mathbf{r}}^{-}\right)\right|$and $h=\left|\left\{t \in\left(G_{\mathbf{r}} \backslash\left(G_{\mathbf{r}}^{+} \cup G_{\mathbf{r}}^{-}\right)\right): t[J]=j\right\}\right|$. In this case, the upper bound of the complexity at the current step of computation drops from $\mathcal{O}\left(2^{H}\right)$ to $\mathcal{O}\left(2^{H-h}+2^{h}\right)$.

The second optimization allows us to prune a subtree of computation even before a contradiction arises. It verifies, in many cases, whether every possible solution that may be built starting from the current partial one turns to be not minimal. Suppose that there exists an active o-pair $\left(t, t^{\prime}\right)$ in a partial solution $\left(G_{\mathbf{r}}, G_{\mathbf{r}}^{+}, G_{\mathbf{r}}^{-}\right)$, such that there exists $\bar{t} \in G_{\mathbf{r}}$ in the same $J$-group of $t$ with $t[V T]<\bar{t}[V T]<t^{\prime}[V T]$. By definition of active o-pair, we have that $\bar{t}$ belongs to $G_{\mathrm{r}}^{-}$as well as every tuple $\bar{t}^{\prime}$ in the same $J$-group of $t$ with $t[V T]<\bar{t}^{\prime}[V T]<t^{\prime}[V T]$. Here, the additional condition is that there exists at least one of such tuples. Given a partial solution $\left(G_{\mathbf{r}}, G_{\mathbf{r}}^{+}, G_{\mathbf{r}}^{-}\right)$, we define set $\operatorname{colors}\left(G_{\mathbf{r}}, G_{\mathbf{r}}^{+}, G_{\mathbf{r}}^{-}\right)=\left\{(x, y, z):\right.$ there exists an active edge $\left(t, t^{\prime}\right)$ with $\left.c\left(t, t^{\prime}\right)=(x, y, z)\right\}$. Let us define the set of colors pending $\left(G_{\mathbf{r}}, G_{\mathbf{r}}^{+}, G_{\mathbf{r}}^{-}\right)=\left\{(x, y, z)\right.$ : there exists a pending edge $\left(t, t^{\prime}\right)$ with $\left.c\left(t, t^{\prime}\right)=(x, y, z)\right\}$, which collects all and only the colors that may be introduced later on in the current computation.

A color $(x, y, z)$ is safe in $\left(v t, v t^{\prime}, j\right)$ if and only if one of the following three conditions hold:

1. $(x, y, z) \in \operatorname{colors}\left(G_{\mathbf{r}}, G_{\mathbf{r}}^{+}, G_{\mathbf{r}}^{-}\right)$;

2. Every color $\left(x, y, z^{\prime}\right) \in \operatorname{pending}\left(G_{\mathbf{r}}, G_{\mathbf{r}}^{+}, G_{\mathbf{r}}^{-}\right)$satisfies $z^{\prime}=z$ (i.e., $(x, y, z)$ is a pending color and there is no pending color that is conflicting with $(x, y, z))$;

3. The color is not conflicting with any color in $\operatorname{colors}\left(G_{\mathbf{r}}, G_{\mathbf{r}}^{+}, G_{\underline{\mathbf{r}}}^{-}\right) \cup \operatorname{pending}\left(G_{\mathbf{r}}, G_{\mathbf{r}}^{+}, G_{-\prime}^{-}\right)$and do not exist two tuples $\bar{t}, \bar{t}^{\prime} \in\left(G_{\mathbf{r}}^{+} \cup G_{\mathbf{r}}^{-}\right) \cap\left\{\bar{t}^{\prime \prime} \in G_{\mathbf{r}}: \bar{t}^{\prime \prime}[J]=\right.$ $\left.j \wedge v t \leq \bar{t}^{\prime \prime} \leq v t^{\prime}\right\}$, such that $\left(\bar{t}, \bar{t}^{-1}\right)$ is an edge and the color $\left.\bar{t}[X], \bar{t}^{\prime}[Y], \bar{t}^{\prime}[Z]\right)$ is conflicting with $(x, y, z)$.

The three conditions above imply that if a color is safe in $\left(v t, v t^{\prime}, j\right)$, then it is neither in conflict with a color in 
Fig. 14 Auxiliary procedures used by procedure TupleWiseMin (Fig. 15)

procedure GroupIndependent? $\left(j, G_{\mathbf{r}}, k, G_{\mathbf{r}}^{+}, G_{\mathbf{r}}^{-}, \mathcal{C}\right)$

returns true if and only if in the current partial solution pending edges

comment: involving tuples belonging to the $J$-group with value $j$ are not conflicting with the pending edges introduced by other $J$-groups.

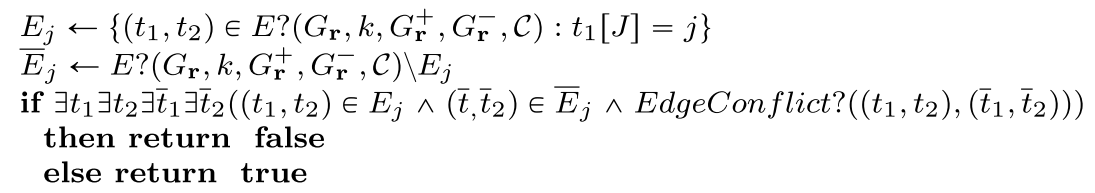

procedure MaxConsistentSubset $(E)$

returns the maximal subset $E^{\prime}$ of $E$ such that for every edge $\left(t_{1}^{\prime}, t_{2}^{\prime}\right) \in E^{\prime}$ comment: and for every edge $\left(t_{1}, t_{2}\right) \in E$ we have that $c\left(t_{1}^{\prime}, t_{2}^{\prime}\right)$ and $c\left(t_{1}, t_{2}\right)$ are not conflicting.

$E^{\prime} \leftarrow \varnothing$

for each $\left(t_{1}, t_{2}\right) \in E$ do $\left\{\begin{aligned} \text { if } \forall \bar{t}_{1} \forall \bar{t}_{2}\left(\left(\bar{t}_{1}, \bar{t}_{2}\right)\right. & \left.\in E \rightarrow \neg \text { EdgeConflict? }\left(\left(t_{1}, t_{2}\right),\left(\bar{t}_{1}, \bar{t}_{2}\right)\right)\right) \\ \text { then } E^{\prime} \leftarrow E^{\prime} & \cup\left\{\left(t_{1}, t_{2}\right)\right\}\end{aligned}\right.$ return $E^{\prime}$

procedure REACH? $\left(t_{1}, t_{2}\right.$, Nodes, Edges $)$

returns true if and only if there exists a path from $t_{1}$ to $t_{2}$ in the graph comment: (Nodes, Edges). It is a function that checks whether there exists a path between two nodes in a graph or not .

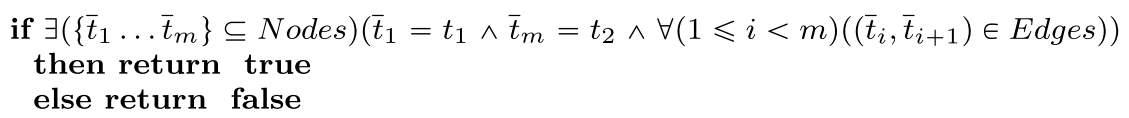
valid times $t[V T]$ and $\bar{t}[V T]$ in $t[J]$-group may be safely replaced.

$\operatorname{colors}\left(G_{\mathbf{r}}, G_{\mathbf{r}}^{+}, G_{\mathbf{r}}^{-}\right)$nor with a color in pending $\left(G_{\mathbf{r}}, G_{\mathbf{r}}^{+}\right.$, $\left.G_{\mathrm{r}}^{-}\right)$. However, this is just a necessary but not sufficient condition. Let us consider the example shown in Fig. 16 and assume that $k \geq 7$ (i.e., every o-pair in the example is also an edge). We have that the active edges are $\left(t_{1}, t_{2}\right),\left(t_{2}, t_{3}\right),\left(t_{3}\right.$, $\left.t_{4}\right)$, and $\left(t_{4}, t_{5}\right)$ for the $j_{1}$-group and $\left(t_{7}, t_{12}\right),\left(t_{8}, t_{12}\right)$ for the $j_{2}$-group, since we have $t_{9}, t_{10}, t_{11} \in G_{\mathbf{r}}^{-}$. Thus, we have $\operatorname{colors}\left(G_{\mathbf{r}}, G_{\mathbf{r}}^{+}, G_{\mathbf{r}}^{-}\right)=\left\{\left(x_{1}, y_{4}, z_{4}\right), \quad\left(x_{2}, y_{5}, z_{6}\right),\left(x_{5}\right.\right.$, $\left.\left.y_{6}, z_{6}\right),\left(x_{4}, y_{6}, z_{5}\right),\left(x_{1}, y_{6}, z_{6}\right),\left(x_{2}, y_{6}, z_{6}\right)\right\}$ and, since we have to decide the status of tuple $t_{6}$, we have pending $\left(G_{\mathbf{r}}, G_{\mathbf{r}}^{+}\right.$, 
Fig. 15 The main procedure for a tuple-wise check of APE-FDs. Notice that we use a compact notation for the recursive procedure which is initially called $\operatorname{TupleWiseMin}\left(G_{\mathbf{r}}, k\right)$. Here, when $G_{\mathbf{r}}^{+}, G_{\mathbf{r}}^{-}$and $\mathcal{C}$ are omitted in the procedure call, they get their respective default values specified in the procedure declaration (i.e., $\emptyset$ for each of them in this case)

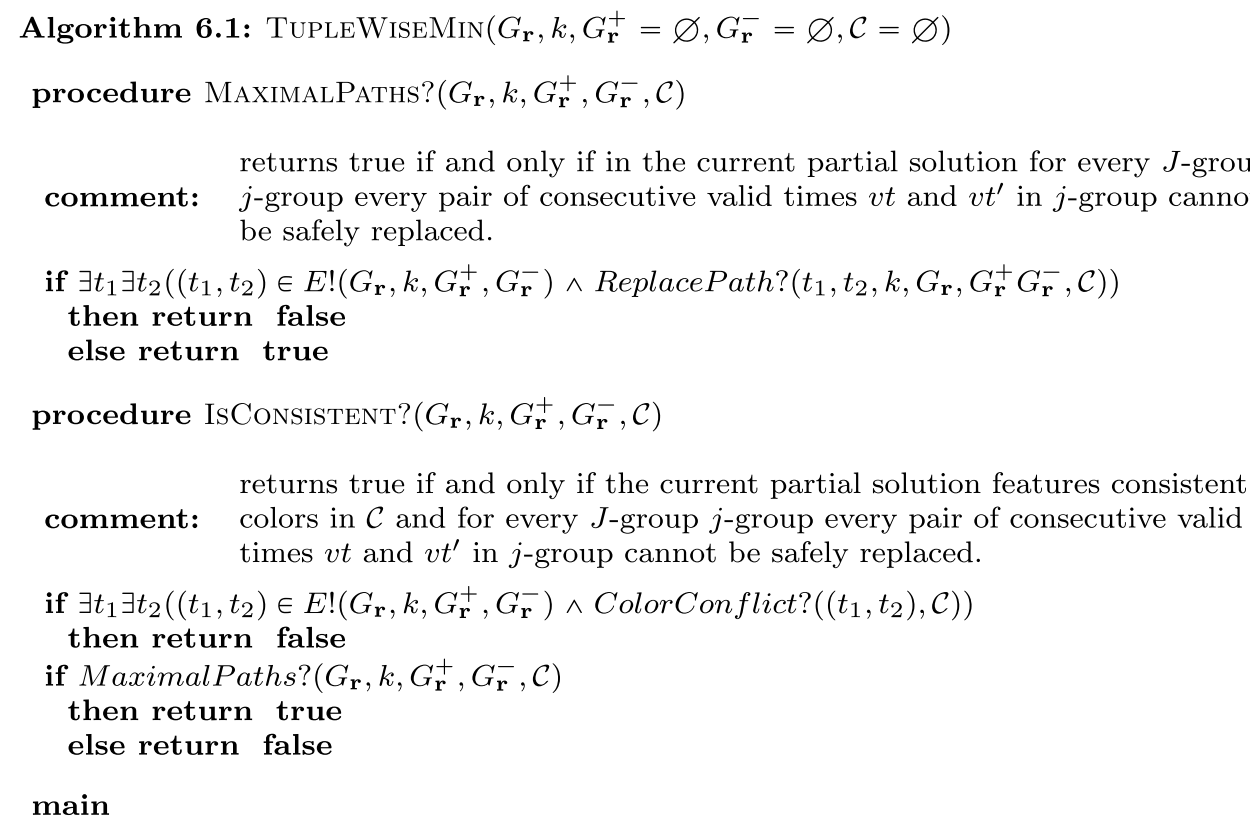

comment:

returns the minimum value $m=\min \left\|G_{\mathbf{r}} \backslash G_{\mathbf{r}}^{\prime}\right\|$ for $G_{\mathbf{r}}^{\prime}$ in $\left\{G_{\mathbf{r}}^{\prime \prime} \subseteq G_{\mathbf{r}}: G_{\mathbf{r}}^{\prime} \models\left[\tau_{G_{\mathbf{r}}}^{X Y Z_{\text {count }}}\right] X \bar{Y} \rightarrow \bar{Z}\right\}$.

if $G_{\mathbf{r}}=\varnothing$ then return $\left\|G_{\mathbf{r}}^{-}\right\|$

if $\exists j\left(j \in \operatorname{Dom}(J) \wedge\right.$ GroupIndependent? $\left.\left(j, G_{\mathbf{r}}, k, G_{\mathbf{r}}^{+}, G_{\mathbf{r}}^{-}, \mathcal{C}\right)\right)$

then $\left\{\begin{array}{l}\bar{G}_{\mathbf{r}} \leftarrow\left\{t \in G_{\mathbf{r}}: t[J]=j\right\} \\ \operatorname{return}\left(\begin{array}{c}\text { TupleWiseCheck }\left(\bar{G}_{\mathbf{r}}, k, G_{\mathbf{r}}^{+} \cap \bar{G}_{\mathbf{r}}, G_{\mathbf{r}}^{-} \cap \bar{G}_{\mathbf{r}}, \mathcal{C}\right)+ \\ \text { TupleWiseCheck }\left(G_{\mathbf{r}} \backslash \bar{G}_{\mathbf{r}}, k, G_{\mathbf{r}}^{+} \backslash \bar{G}_{\mathbf{r}}, G_{\mathbf{r}}^{-} \backslash \bar{G}_{\mathbf{r}}, \mathcal{C}\right)\end{array}\right)\end{array}\right.$

let $t \in G_{\mathbf{r}}$

if IsConsistent? $\left(G_{\mathbf{r}} \backslash\{t\}, k, G_{\mathbf{r}}^{+} \cup\{t\}, G_{\mathbf{r}}^{-}, \mathcal{C}\right)$

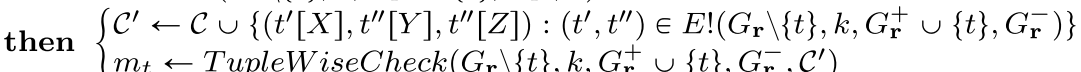

else $m_{t} \leftarrow+\infty$

if IsConsistent? $\left(G_{\mathbf{r}} \backslash\{t\}, k, G_{\mathbf{r}}^{+}, G_{\mathbf{r}}^{-} \cup\{t\}, \mathcal{C}\right)$

then $\left\{\begin{array}{l}\mathcal{C}^{\prime} \leftarrow \mathcal{C} \cup\left\{\left(t^{\prime}[X], t^{\prime \prime}[Y], t^{\prime \prime}[Z]\right):\left(t^{\prime}, t^{\prime \prime}\right) \in E !\left(G_{\mathbf{r}} \backslash\{t\}, k, G_{\mathbf{r}}^{+}, G_{\mathbf{r}}^{-} \cup\{t\}\right)\right\} \\ m_{女} \leftarrow \text { TupleWiseCheck }\left(G_{\mathbf{r}} \backslash\{t\}, k, G_{\mathbf{r}}^{+}, G_{\mathbf{r}}^{-} \cup\{t\}, \mathcal{C}^{\prime}\right)\end{array}\right.$

else $m_{t} \leftarrow+\infty$

return $\min \left(m_{t}, m_{\xi}\right)$
$\left.G_{\mathbf{r}}^{-}\right)=\left\{\left(x_{1}, y_{3}, z_{2}\right)\right\}$. Suppose that we are interested in the colors which are safe in $\left(1,5, j_{2}\right)$. For instance, $c\left(t_{7}, t_{9}\right)=\left(x_{1}, y_{3}, z_{3}\right)$ (i.e., the dotted edge in the $j_{2}$-group in Fig. 16) is not safe in $\left(1,5, j_{2}\right)$ because it does not belong neither to $\operatorname{colors}\left(G_{\mathbf{r}}, G_{\mathbf{r}}^{+}, G_{\mathbf{r}}^{-}\right)$nor to pending $\left(G_{\mathbf{r}}, G_{\mathbf{r}}^{+}, G_{\mathbf{r}}^{-}\right)$ (conditions 1 and 2) and it is in conflict with the unique color $\left(x_{1}, y_{3}, z_{2}\right) \in$ pending $\left(G_{\mathbf{r}}, G_{\mathbf{r}}^{+}, G_{\mathbf{r}}^{-}\right)$. On the other hand, colors $c\left(t_{8}, t_{9}\right)=\left(x_{2}, y_{3}, z_{3}\right), c\left(t_{8}, t_{10}\right)=\left(x_{2}, y_{4}, z_{4}\right)$ and $c\left(t_{10}, t_{11}\right)=\left(x_{4}, y_{5}, z_{5}\right)$ (i.e., the dashed edges in Fig. 16) are safe in $\left(1,5, j_{2}\right)$, because they are neither in conflict with colors either in colors $\left(G_{\mathbf{r}}, G_{\mathbf{r}}^{+}, G_{\mathbf{r}}^{-}\right)$or in pending $\left(G_{\mathbf{r}}, G_{\mathbf{r}}^{+}, G_{\mathbf{r}}^{-}\right)$ and there are no other edges in $j_{2}$ with valid times between 1 and 5 that exhibit either $\left(x_{2}, y_{3}\right)$, or $\left(x_{4}, y_{5}\right)$, or $\left(x_{2}, y_{3}\right)$ as first two components of their colors (thus condition 3 applies to these colors). Colors $c\left(t_{7}, t_{10}\right)=\left(x_{1}, y_{4}, z_{4}\right)$ and $c\left(t_{11}, t_{12}\right)=\left(x_{5}, y_{6}, z_{6}\right)$ (i.e., the continuous edges in $j_{2^{-}}$ group in Fig. 16) are safe in $\left(1,5, j_{2}\right)$, because they belong to $\operatorname{colors}\left(G_{\mathbf{r}}, G_{\mathbf{r}}^{+}, G_{\mathbf{r}}^{-}\right)$and thus both satisfy condition 1 . Finally, colors $c\left(t_{8}, t_{11}\right)=\left(x_{2}, y_{5}, z_{5}\right)$, and $c\left(t_{8}, t_{10}\right)=\left(x_{4}, y_{6}, z_{6}\right)$ are not safe in $\left(1,5, j_{2}\right)$ (i.e., the $X$-labeled edges in Fig. 16), because they are in conflict with two colors in $\operatorname{colors}\left(G_{\mathbf{r}}, G_{\mathbf{r}}^{+}, G_{\mathbf{r}}^{-}\right)$; more precisely, $\left(x_{2}, y_{5}, z_{5}\right)$ is in conflict with $\left(x_{2}, y_{5}, z_{6}\right)$ and $\left(x_{4}, y_{6}, z_{6}\right)$ is in conflict with $\left(x_{4}, y_{6}, z_{5}\right)$.

Given a partial solution $\left(G_{\mathbf{r}}, G_{\mathbf{r}}^{+}, G_{\mathbf{r}}^{-}\right)$and the triple $\left(v t, v t^{\prime}, j\right)$, a $\left(v t, v t^{\prime}, j\right)$-replace $D A G$ is a DAG $(V, E)$ where $V=\left\{t \in G_{\mathbf{r}}^{+}: t[V T]=v t \wedge t[J]=j\right\} \cup\left\{t \in G_{\mathbf{r}}^{-}: v t<t\right.$ $\left.[V T]<v t^{\prime} \wedge t[J]=j\right\} \cup\left\{t \in G_{\mathbf{r}}^{+}: t[V T]=v t^{\prime} \wedge t[J]=j\right\}$ and 
Fig. 16 An example of how a partial solution may be improved
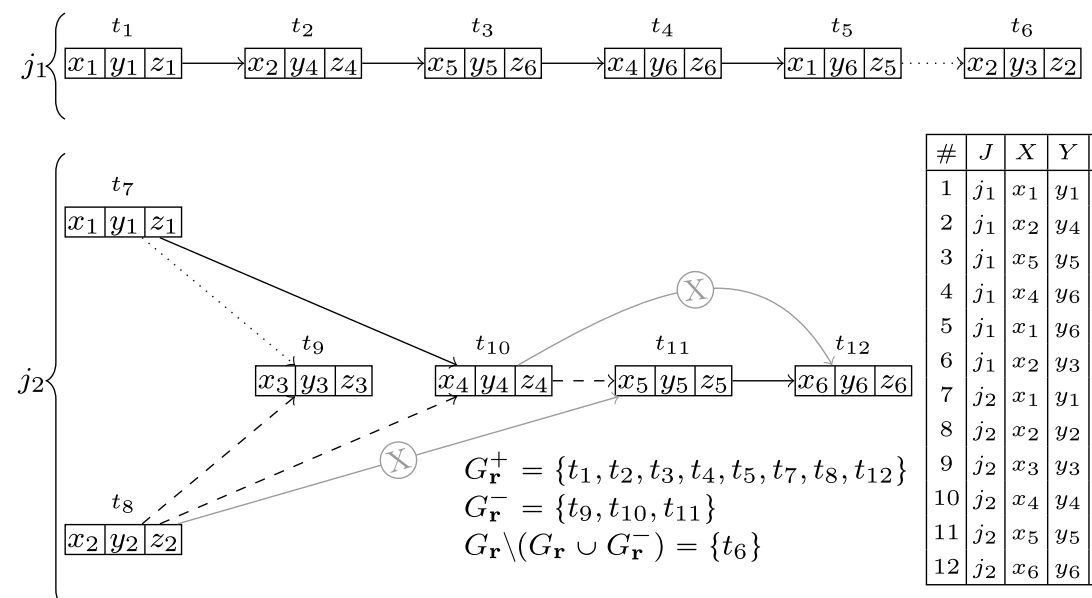

\begin{tabular}{|c|c|c|c|c|c|c|}
\hline$\#$ & $J$ & $X$ & $Y$ & $Z$ & $V T$ & count \\
\hline 1 & $j_{1}$ & $x_{1}$ & $y_{1}$ & $z_{1}$ & 2 & 1 \\
2 & $j_{1}$ & $x_{2}$ & $y_{4}$ & $z_{4}$ & 4 & 1 \\
3 & $j_{1}$ & $x_{5}$ & $y_{5}$ & $z_{6}$ & 5 & 1 \\
4 & $j_{1}$ & $x_{4}$ & $y_{6}$ & $z_{6}$ & 6 & 1 \\
5 & $j_{1}$ & $x_{1}$ & $y_{6}$ & $z_{5}$ & 7 & 1 \\
6 & $j_{1}$ & $x_{2}$ & $y_{3}$ & $z_{2}$ & 6 & 1 \\
7 & $j_{2}$ & $x_{1}$ & $y_{1}$ & $z_{1}$ & 1 & 1 \\
8 & $j_{2}$ & $x_{2}$ & $y_{2}$ & $z_{2}$ & 2 & 1 \\
9 & $j_{2}$ & $x_{3}$ & $y_{3}$ & $z_{2}$ & 3 & 1 \\
10 & $j_{2}$ & $x_{4}$ & $y_{4}$ & $z_{3}$ & 4 & 1 \\
11 & $j_{2}$ & $x_{5}$ & $y_{5}$ & $z_{4}$ & 5 & 1 \\
12 & $j_{2}$ & $x_{6}$ & $y_{6}$ & $z_{5}$ & 6 & 1 \\
\hline
\end{tabular}

$$
E=\begin{gathered}
\left\{\left(t, t^{\prime}\right) \in V \times V: \begin{array}{c}
\left(t[V T] \neq v t \vee t^{\prime}[V T] \neq v t^{\prime}\right) \wedge t[V T]<t^{\prime}[V T] \wedge \\
c\left(t, t^{\prime}\right) \text { is safe in }\left(v t, v t^{\prime}, j\right)
\end{array}\right\} \\
\cup \\
\left\{\left(t, t^{\prime}\right) \in V \times V:\left(t[V T] \neq v t \vee t^{\prime}[V T] \neq v t^{\prime}\right) \wedge t^{\prime}[V T]-t[V T]>k\right\}
\end{gathered}
$$

A node $t \in V$ is a starting node (resp. ending node) if and only if $v t<t[V T]<v t^{\prime}$ and, for every $t^{\prime} \in V$ with $t^{\prime}[V T]=v t$ (resp. $\left.t^{\prime}[V T]=v t^{\prime}\right)$, we have $\left(t^{\prime}, t\right) \in E$ (resp. $\left(t, t^{\prime}\right) \in E$ ). A replace path in a $\left(v t, v t^{\prime}, j\right)$-replace DAG $(V, E)$ is any path $t_{1} \ldots t_{m}$ in $(V, E)$ for which $t_{1}$ is a starting node and $t_{m}$ is an ending node. We say that $v t$ and $v t^{\prime}$ in $j$ can be safely replaced if and only if there exists a replace path in the $\left(v t, v t^{\prime}, j\right)$ -replace DAG $(V, E)$. Figure 16 depicts the $\left(1,5, j_{2}\right)$-replace DAG, where $t_{10}$ is the only initial node that is not an ending one, and $t_{11}$ is the only ending node that is not a starting one. Since $t_{10}$ is connected to $t_{11}$, we have that $t_{10} t_{11}$ is a replace path in the $\left(1,5, j_{2}\right)$-replace DAG and thus 1 and 5 can be safely replaced in $j_{2}$. Using the above definitions of replace DAGs/paths, we can provide the following result.

Theorem 3 Given a partial solution $\left(G_{\mathbf{r}}, G_{\mathbf{r}}^{+}, G_{\mathbf{r}}^{-}\right)$, if there exists a group $j$ with two consecutive valid times $v t$ and $v t^{\prime}$ such that $v t$ and $v t^{\prime}$ can be safely replaced in $j$, then every consistent solution that follows $\left(G_{\mathbf{r}}, G_{\mathbf{r}}^{+}, G_{\mathbf{r}}^{-}\right)$is not optimal.

The proof of the theorem is straightforward. Let us suppose that $t_{1} \ldots t_{m}$ is a replace path in the $\left(v t, v t^{\prime}, j\right)$-replace DAG. By definition, we have $t_{1}, \ldots, t_{m} \in G_{\mathrm{r}}^{-}$. It suffices to take any consistent solution $\left(G_{\mathbf{r}}, \bar{G}_{\mathbf{r}}^{+}, \bar{G}_{\mathbf{r}}^{-}\right)$that follows $\left(G_{\mathbf{r}}, G_{\mathbf{r}}^{+}, G_{\mathbf{r}}^{-}\right)$ and such that $\left(G_{\mathbf{r}}, \bar{G}_{\mathbf{r}}^{+} \cup\left\{t_{1}, \ldots, t_{m}\right\}, \bar{G}_{\mathbf{r}}^{-} \backslash\left\{t_{1}, \ldots, t_{m}\right\}\right)$ is still a consistent solution. Nonoptimality immediately follows.

We take advantage of Theorem 3 by pruning every computation rooted in a partial solution $\left(G_{\mathbf{r}}, G_{\mathbf{r}}^{+}, G_{\mathbf{r}}^{-}\right)$that features a $J$-group $j$-group and two consecutive valid times $v t$ and $v t^{\prime}$ in the $j$-group, such that $v t$ and $v t^{\prime}$ can be safely replaced in $j$. Verifying whether such condition applies or not may be performed in polynomial time. In procedure TupleWiseMin, this optimization is realized by subprocedures Maximal Paths? and Replace Path? reported in Figs. 14 and 15, respectively.

\section{The Second Algorithm}

Let us now propose another algorithm with some auxiliary procedures, reported in Figs. 17 and 18, for solving problem Check-APE-FD. Such an algorithm, whose main procedure is called EdgeWiseMin, strongly differs from TupleWiseMin in approaching the problem. In principle, it works better, but it may work only under a quite reasonable assumption on the input, which we will discuss in detail later on.

At every step, procedure EdgeWiseMin, instead of guessing if a tuple belongs to the current partial solution, guesses if a color is forbidden or allowed in the current partial solution. Informally, forbidding a color $(x, y, z)$ means avoiding all the active edges $\left(t, t^{\prime}\right) \in G_{\mathbf{r}} \times G_{\mathbf{r}}$ for which $c\left(t, t^{\prime}\right)=(x, y, z)$. On the other hand, allowing a color $(x, y, z)$ means forbidding all the active edges $\left(t, t^{\prime}\right) \in G_{\mathbf{r}} \times G_{\mathbf{r}}$ whose colors are conflicting with $(x, y, z)$. In order to do that, we introduce the concept of color-partial solution. A color-partial solution is a triple $\left(G_{\mathbf{r}}, \mathcal{C}^{+}, \mathcal{C}^{-}\right)$, such that $\mathcal{C}^{+}, \mathcal{C}^{-} \subseteq \operatorname{Dom}(X) \times \operatorname{Dom}(Y) \times \operatorname{Dom}(Z)$ are disjoint subsets of colors (i.e., $\mathcal{C}^{+} \cap \mathcal{C}^{-}=\emptyset$ ) and for every pair of colors $(x, y, z),\left(x^{\prime}, y^{\prime}, z^{\prime}\right) \in \mathcal{C}^{+}(x, y, z)$ is not conflicting with $\left(x^{\prime}, y^{\prime}, z^{\prime}\right)$ (i.e., if $x^{\prime}=x$ and $y^{\prime}=y$ then $z^{\prime}=z$ ). 
Fig. 17 Auxiliary procedures for the main ones in Fig. 18. Procedure BuildDag builds a single-source, single-sink DAG whose nodes are nonempty subsets of $G_{\mathbf{r}}$. Each subset is formed by tuples sharing the same value for $V T$, and thus function Time is well defined. Procedure SourceSinkShortestPath returns the shortest path from source to sink on the DAG provided by BuildDag. The solution is given as a set of nodes (i.e., subsets of $G_{\mathbf{r}}$ ), and it omits source and sink nodes procedure SourceSinkShortestPath(Nodes, Edges, Time, Weight)

returns the shortest path from a source node to a sink node in a Weighted DAG (Nodes, Edges). The weight of each edge is provided by the function Weight. The topological order of the elements of Nodes is provided by the function Time whch is used for identifying the source (Time(source) = $-\infty)$ and the sink (Time $(\operatorname{sink})=+\infty)$ nodes. Finally, the shortest path is returned as a subset of Nodes.

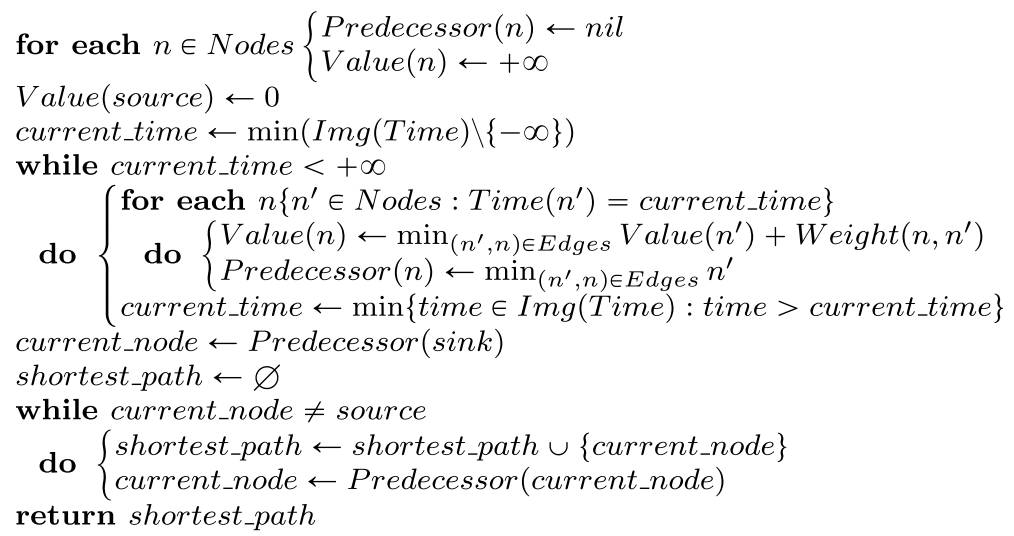

procedure BuILdDAG $\left(G_{\mathbf{r}}, k, \mathcal{C}^{+}, \mathcal{C}^{-}\right)$

given a set $G_{\mathbf{r}}$ for which there exists $j \in \operatorname{Dom}(J)$ such that for every $t \in G_{\mathbf{r}}$ comment: $\quad$ we have $t[J]=j$. Let $C P S=\left(G_{\mathbf{r}}, \mathcal{C}^{+}, \mathcal{C}^{-}\right)$The procedure returns the unfolded DAG for $\mathcal{L}_{C P S}^{j}$.

Nodes $\leftarrow\left\{S \subseteq G_{\mathbf{r}}: S \neq \varnothing \wedge \forall t \forall t^{\prime}\left(t \in S \wedge t^{\prime} \in S \rightarrow t[V T]=t^{\prime}[V T]\right)\right\}$

EndNodes $\leftarrow\{$ source, sink\}

AllNodes $=$ Nodes $\cup$ EndNodes

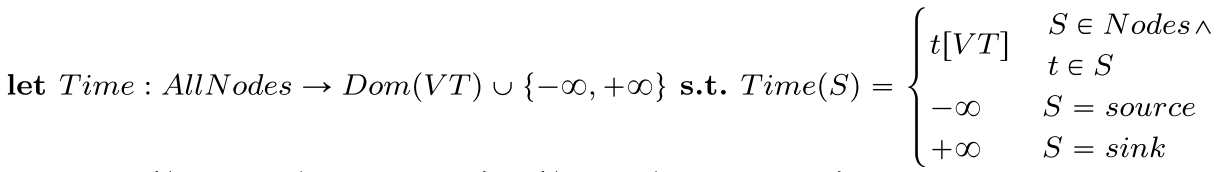

Edges $\leftarrow \begin{array}{ll}\{(\text { source }, S): S \in N \text { odes }\} \cup\{(S, \text { sink }): S \in N o d e s\} \\ \cup\left\{\left(S, S^{\prime}\right): S \in N \text { odes }, S^{\prime} \in N \text { odes }, \text { Time }\left(S^{\prime}\right)-\text { Time }(S)>k\right\}\end{array}$

Edges $\leftarrow\left\{\left(S, S^{\prime}\right): \forall t \forall t^{\prime}\left(\left(\begin{array}{l}t \in S \wedge t^{\prime} \in S^{\prime} \wedge \\ t^{\prime}[V T]-t[V T]>0\end{array}\right) \rightarrow\left(\begin{array}{l}\neg \operatorname{Color} \operatorname{Conflict}\left(\left(t[X], t^{\prime}[Y], t^{\prime}[Z]\right), \mathcal{C}^{+}\right) \\ \wedge\left(t[X], t^{\prime}[Y], t^{\prime}[Z]\right) \notin \mathcal{C}^{-}\end{array}\right)\right)\right\}$

let Weight : Edges $\rightarrow \mathbb{N}$ s.t.

$W \operatorname{Weight}\left(S, S^{\prime}\right)= \begin{cases}\sum_{t \in G_{\mathbf{r}}: \operatorname{Time}(S)<t[V T] \leqslant \operatorname{Time}\left(S^{\prime}\right) \wedge t \notin S^{\prime}}\|t\| & \text { if } S, S^{\prime} \in \text { Nodes } \\ \sum_{t \in G_{\mathbf{r}}: t[V T] \leqslant \operatorname{Time}\left(S^{\prime}\right) \wedge t \notin S^{\prime}}\|t\| & \text { if } S=\text { source } \\ \sum_{t \in G_{\mathbf{r}}: t[V T]>\operatorname{Time}(S) \wedge t \notin S^{\prime}}\|t\| & \text { if } S^{\prime}=\text { sink }\end{cases}$

return (Nodes $\cup$ EndNodes, Edges, Time, Weight)
Let $C P S=\left(G_{\mathbf{r}}, \mathcal{C}^{+}, \mathcal{C}^{-}\right)$be a color-partial solution: we say that a solution $\left(G_{\mathbf{r}}, G_{\mathbf{r}} \backslash G_{\mathbf{r}}^{-}, G_{\mathbf{r}}^{-}\right)$is induced by $C P S$ if and only if the two following conditions hold:

1. for every color $(x, y, z)$ in $\mathcal{C}^{+}$and for each edge $\left(t, t^{\prime}\right) \in G_{\mathbf{r}} \times G_{\mathbf{r}}$ for which $c\left(t, t^{\prime}\right)=\left(x, y, z^{\prime}\right)$, if $\left(t, t^{\prime}\right)$ is active then $z^{\prime}=z$.

2. for every color $(x, y, z)$ in $\mathcal{C}^{-}$and for each edge $\left(t, t^{\prime}\right) \in G_{\mathbf{r}} \times G_{\mathbf{r}}$, if $c\left(t, t^{\prime}\right)=(x, y, z)$ then $\left(t, t^{\prime}\right)$ is not active in $\left(G_{\mathbf{r}}, G_{\mathbf{r}}^{+}, G_{\mathbf{r}}^{-}\right)$. It means that one of the following two conditions holds:

- $t \in G_{\mathbf{r}}^{-}$or $t^{\prime} \in G_{\mathbf{r}}^{-}$;
- there exists a tuple $t^{\prime \prime} \in G_{\mathrm{r}}^{+}$such that $t[V T]<t^{\prime \prime}[V T]<t^{\prime}[V T]$ and $t^{\prime \prime}[J]=t[J](t[V T]$ and $t^{\prime}[V T]$ are not consecutive in the current partial solution for the $J$-group with value $t[J])$.

An induced solution $\left(G_{\mathbf{r}}, G_{\mathbf{r}} \backslash G_{\mathbf{r}}^{-}, G_{\mathbf{r}}^{-}\right)$by $C P S$ is minimal if and only if an induced solution $\left(G_{\mathbf{r}}, G_{\mathbf{r}} \backslash \bar{G}_{\mathbf{r}}^{-}, \bar{G}_{\mathbf{r}}^{-}\right)$by $C P S$ does not exist with $\left|G_{\mathrm{r}}^{-}\right|>\left|\bar{G}_{\mathrm{r}}^{-}\right|$. In this case, we say that $\left(G_{\mathbf{r}}, G_{\mathbf{r}} \backslash G_{\mathbf{r}}^{-}, G_{\mathbf{r}}^{-}\right)$is an induced minimal solution. Since all the induced minimal solutions by $C P S$ have the same size, we say that the value of CPS, denoted by val(CPS), is the value $\left\|G_{\mathbf{r}}^{-}\right\|$, where $\left(G_{\mathbf{r}}, G_{\mathbf{r}} \backslash G_{\mathbf{r}}^{-}, G_{\mathbf{r}}^{-}\right)$is a minimal induced partial solution. 
Fig. 18 Main procedure for the edge-wise checking of a $A P E$ -FD $\left[\Delta_{k}\left(\tau_{G}^{X Y Z \text { count }}\right)\right] X \bar{Y} \stackrel{\epsilon}{\rightarrow} \bar{Z}$. The procedure returns the minimum number of tuples to delete in $\mathbf{r}$ in order to obtain an instance $\mathbf{r}^{\prime} \subseteq \mathbf{r}$ such that $\mathbf{r}^{\prime} \vDash\left[\Delta_{k}\left(\tau_{\mathbf{r}}^{R}\right)\right] X \overline{\bar{Y}} \rightarrow \bar{Z}$. Like procedure TupleWiseMin of Figure 15, the initial call to the recursive procedure is $\operatorname{EdgeWiseMin}\left(G_{\mathbf{r}}, k\right)$ with $\mathcal{C}^{+}$, $\mathcal{C}^{-}$, and optimal initialized to their respective default values

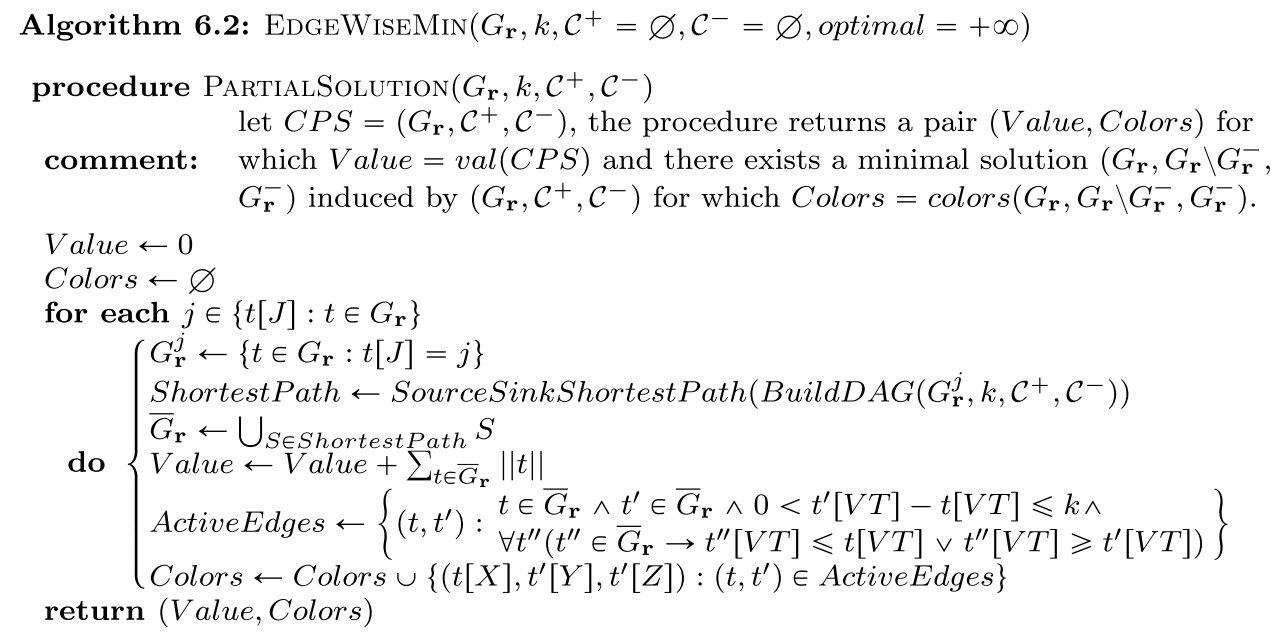

main

returns the minimum value $m=\min \left\|G_{\mathbf{r}} \backslash G_{\mathbf{r}}^{\prime}\right\|$

comment: for $G_{\mathbf{r}}^{\prime}$ in $\left\{G_{\mathbf{r}}^{\prime \prime} \subseteq G_{\mathbf{r}}: G_{\mathbf{r}}^{\prime} \models\left[\tau_{G_{\mathbf{r}}}^{X Y Z \text { count }}\right] X \bar{Y} \rightarrow \bar{Z}\right\}$.

$m \leftarrow 0$

Colors $\leftarrow \varnothing$

for each $g \in\left\{g: \exists\left(t \in G_{\mathbf{r}}\right)(t[J]=g)\right\}$

do $\left\{\begin{array}{l}(\text { Value }, C) \leftarrow \text { PartialSolution }\left(G b r, k, \mathcal{C}^{+}, \mathcal{C}^{-}\right) \\ m \leftarrow m+\text { Value } \\ \text { Colors } \leftarrow \text { Colors } \cup C\end{array}\right.$

if $m \geqslant$ optimal then return optimal

for each $c \in$ Colors $\left\{\begin{aligned} \text { for each } c^{\prime} \in \text { Colors } \\ \text { return } m\end{aligned}\left\{\begin{aligned} \text { if } c[X]=c^{\prime}[X] \wedge c[\bar{Y}]=c^{\prime}[\bar{Y}] \wedge c[\bar{Z}] \neq c^{\prime}[\bar{Z}] \\ \text { then }\left\{\begin{array}{l}m_{c} \leftarrow \text { EdgeWiseMin }\left(G_{\mathbf{r}}, k, \mathcal{C}^{+} \cup\{c\}, \mathcal{C}^{-}, \text {optimal }\right) \\ m_{\neg c} \leftarrow \text { EdgeWiseMin }\left(G_{\mathbf{r}}, k, \mathcal{C}^{+}, \mathcal{C}^{-} \cup\{c\}, \text { optimal }\right) \\ m \leftarrow \min \left(m_{c}, m_{\neg c}\right) \\ \text { return min }(m, \text { optimal })\end{array}\right.\end{aligned}\right.\right.$

In an opposite way from TupleWiseMin, in this algorithm color-partial solutions induce complete minimal solutions. However, such solutions may be inconsistent. The algorithm tries to obtain consistency by either forcing or forbidding one color at a time. This is done by means of sets $\mathcal{C}^{+}$and $\mathrm{C}^{-}$, which are both initialized to $\emptyset$ at the beginning of the procedure. As we informally said above, if a color $(x, y, z)$ belongs to $\mathcal{C}^{+}$, it means that the current partial solution must avoid all the active edges $\left(t, t^{\prime}\right)$ such that $t[X]=x, t^{\prime}[Y]=y$, and $t^{\prime}[Z] \neq z$; if a color $(x, y, z)$ belongs to $\mathcal{C}^{-}$, it means that the current partial solution must avoid all the active edges $\left(t, t^{\prime}\right)$ such that $t[X]=x, t^{\prime}[Y]=y$, and $t^{\prime}[Z]=z$.

As a general overview of the algorithm, let us consider the following simplified procedure (let $C P S=\left(G_{\mathbf{r}}, \mathcal{C}^{+}, \mathcal{C}^{-}\right)$to be the current color-partial solution):

1. compute a minimal solution $\left(G_{\mathbf{r}}, G_{\mathbf{r}} \backslash G_{\mathbf{r}}^{-}, G_{\mathbf{r}}^{-}\right)$induced by CPS;

2. if $\left(G_{\mathbf{r}}, G_{\mathbf{r}} \backslash G_{\mathbf{r}}^{-}, G_{\mathbf{r}}^{-}\right)$is consistent, $\operatorname{return} \operatorname{val}(C P S)=\left\|G_{\mathbf{r}}^{-}\right\|$

3. if $\left(G_{\mathbf{r}}, G_{\mathbf{r}} \backslash G_{\mathbf{r}}^{-}, G_{\mathbf{r}}^{-}\right)$is inconsistent, let $\left(t, t^{\prime}\right)$ be an active edge in $\left(G_{\mathbf{r}}, G_{\mathbf{r}} \backslash G_{\mathbf{r}}^{-}, G_{\mathbf{r}}^{-}\right)$, such that there exists an active edge $\left(t^{\prime \prime}, t^{\prime \prime \prime}\right)$ in $\left(G_{\mathbf{r}}, G_{\mathbf{r}} \backslash G_{\mathbf{r}}^{-}, G_{\mathbf{r}}^{-}\right)$, which is conflicting with $\left(t, t^{\prime}\right)$. Then, return the minimum value between those returned by two recursive calls, one where $\mathcal{C}^{+}$is updated to $\mathcal{C}^{+} \cup\left\{\left(t[X], t^{\prime}[Y], t^{\prime}[Z]\right)\right\}$, and the other one where $\mathcal{C}^{-}$is updated to $\mathcal{C}^{-} \cup\left\{\left(t[X], t^{\prime}[Y], t^{\prime}[Z]\right)\right\}$.

Two observations are omitted in the above procedure w.r.t. function EdgeWiseMin. The first one is that the procedure does not take into account the fact that the value $\left\|G_{\mathbf{r}}^{-}\right\|$of the color-partial solution computed at point 1 is a lower bound for the optimal solution that may be achieved in the current branch of the computation. Procedure EdgeWiseMin uses it in a classical branch-and-bound fashion by propagating the value of the current optimal solution (if any) in the tree of recursive calls (in Fig. 18 this is done by means of parameter optimal). In step 1., if the computed value $\left\|G_{\mathbf{r}}^{-}\right\|$is greater than the optimal one, we immediately return from the recursive call, because no better solution may be found.

The last omitted observation regards how the value of the color-partial solution $\operatorname{val}(C P S)$ is computed, where $C P S=\left(G_{\mathbf{r}}, \mathcal{C}^{+}, \mathcal{C}^{-}\right)$. For every $J$-group in $G_{\mathbf{r}}$, i.e. any set of tuples having value $j$ for attribute $J$, we build 
the following $w L-D A G \mathcal{L}_{C P S}^{j}=\left(V^{j}, E^{j}, \mathcal{L}^{j}, \mathcal{W}^{j}\right)$ where $V^{j}=\left\{t \in G_{\mathbf{r}}: t[J]=j\right\}$. For each $t \in V^{j}$, we have $\mathcal{W}^{j}(t)=t[$ count $]$ and $\mathcal{L}^{j}(t)=t[V T]$, and

$$
\begin{aligned}
& \left\{\left(t, t^{\prime}\right) \in V^{j} \times V^{j}: t^{\prime}[V T]-t[V T]>k\right\} \\
& U \\
& E^{j}=\left\{\left(t, t^{\prime}\right) \in V^{j} \times V^{j}: 0<t^{\prime}[V T]-t[V T] \leq k \wedge c\left(t, t^{\prime}\right) \in \mathcal{C}^{+}\right\} \\
& \left\{\left(t, t^{\prime}\right) \in V^{j} \times V^{j}: \begin{array}{l}
0<t^{\prime}[V T]-t[V T] \leq k \wedge c\left(t, t^{\prime}\right) \notin \mathcal{C}^{-} \wedge \\
\forall x \forall y \forall z\left((x, y, z) \in \mathcal{C}^{+} \rightarrow c\left(t, t^{\prime}\right) \text { is not conflicting with }(x, y, z)\right.
\end{array}\right\}
\end{aligned}
$$

Let $J\left(G_{\mathbf{r}}\right)$ be the set $\left\{j: \exists t\left(t \in G_{\mathbf{r}} \wedge t[J]=j\right)\right\}=\left\{j_{1}, \ldots, j_{h}\right\}$. For every $1 \leq i \leq h$, let $M_{C P S}^{i}$ be the maximum value for which the $w L-D A G \mathcal{L}_{C P S}^{j_{i}}$ admits an $M_{C P S}^{i}$-thick path. The following result is straightforward.

Theorem 4 For every color-partial solution $C P S=\left(G_{\mathbf{r}}, \mathcal{C}^{+}, \mathcal{C}^{-}\right)$we have

$\operatorname{val}(C P S)=\left\|G_{\mathbf{r}}\right\|-\sum_{j\left(G_{\mathbf{r}}\right)} M_{C P S}^{j}$

Theorem 4 tells us that for computing $\operatorname{val}(C P S)$, we need to compute for every $j \in J\left(G_{\mathbf{r}}\right)$ the maximum value $M_{C P S}^{j}$, for which $\mathcal{L}_{C P S}^{j}$ admits an $M_{C P S}^{j}$-thick path. Given a $w L-D A G \mathcal{L}_{\mathcal{G}}$, we will call MAX-ThickPath (Max-TP for short) the problem of finding the maximum $M$ for which $\mathcal{L}_{\mathcal{G}}$ admits a $M$-Thick Path. Max-TP may be solved by a simple dichotomic search having a decision procedure that solves the problem $M$-TP (Problem 4), which is NP-Complete [10]. Here, our assumption comes into play and allows us to find $M_{C P S}^{j}$ for every $j \in J\left(G_{\mathbf{r}}\right)$ in a "reasonable" time. Indeed, in the instances that are used to prove the NP-completeness, the number of nodes in any layer, roughly corresponding to the number of tuples of the given relation at a corresponding time point, is supposed to increase as the number of time points/layers increases.

This is not the case in many daily applications, especially in the clinical domain, where we may have a great number of tuples but scattered along the timeline. In thefollowing, we will provide a formal definition of our assumption. Given $j \in J\left(G_{\mathbf{r}}\right)$ we define $V T\left(G_{\mathbf{r}}, j\right)=\left\{v t: \exists t\left(t \in G_{\mathbf{r}} \wedge t[V T]=v t \wedge t[J]=j\right)\right\}$, $\operatorname{MaxLevel}\left(G_{\mathbf{r}}, j\right)=\max _{v t \in V T\left(G_{\mathbf{r}}, j\right)}|\{t: t[J]=j \wedge t[V T]=v t\}|$, $\left.\operatorname{Max} \operatorname{Count}\left(G_{\mathbf{r}}, j\right)=\max _{v t \in V T\left(G_{\mathbf{r}}, j\right)}\left(\sum_{t \in G_{\mathbf{r}} \wedge t[V T]=v \wedge t[J]=j}\|t\|\right)\right)$ and the value $\operatorname{space}\left(j, G_{\mathbf{r}}\right)$ as the value $2^{\operatorname{MaxLevel}\left(G_{\mathrm{r}}, j\right)}$ $\cdot\left|V T\left(G_{\mathbf{r}}, j\right)\right| \cdot \log _{2}\left(\operatorname{Max} \operatorname{Count}\left(G_{\mathbf{r}}, j\right)\right)$. Let $\operatorname{MaxSpace}\left(G_{\mathbf{r}}\right)$ $=\max _{j \in J\left(G_{\mathbf{r}}\right)} \operatorname{space}\left(j, G_{\mathbf{r}}\right)$. We will see that EdgeWiseMin is applicable to our instance, if we have $\mathcal{O}\left(\operatorname{MaxSpace}\left(G_{\mathbf{r}}\right)^{2}\right)$ bits for computing it. The problem with $\operatorname{MaxPace}\left(G_{\mathbf{r}}\right)$ is that it is exponential in $\operatorname{MaxLevel}\left(G_{\mathbf{r}}, j\right)$, but this value depends on the maximum number of tuples that shares the same values for attributes $V T$ and $J$ and differs on at least one among the attributes $X, Y$ and $Z$ in the original instance r. As we say above, we assume this value to be manageable as it happens in many real-world applications. Hereinafter, we will suppose to have $\mathcal{O}\left(\operatorname{MaxSpace}\left(G_{\mathbf{r}}\right)^{2}\right)$ bits for performing our computation.

Let us suppose to have a $w L-D A G \mathcal{L}_{\mathcal{G}}=(V, E, \mathcal{L}, \mathcal{W})$, and we want to solve MAX-TP on it. Given a $w L-D A G$ $\mathcal{L}_{\mathcal{G}}=(V, E, \mathcal{L}, \mathcal{W})$, a subset $V^{\prime} \subseteq V$ is a level-subset if and only if $V^{\prime} \neq \emptyset$ and $\mathcal{L}(v)=\mathcal{L}\left(v^{\prime}\right)$ for every $v, v^{\prime} \in V^{\prime}$. Let $\mathcal{V}=\left\{V^{\prime} \subseteq V: V^{\prime}\right.$ is a level-subset $\}$. By definition of level subset, the function $\mathcal{L}^{\prime}: \mathcal{V} \rightarrow \mathbb{N}$, such that for every $V^{\prime} \in \mathcal{V}$ we have $\mathcal{L}^{\prime}\left(V^{\prime}\right)=\mathcal{L}(v)$ for some $v \in V^{\prime}$, turns out to be well defined. We define the unfolding of $w L-D A G \quad \mathcal{L}_{\mathcal{G}}=(V, E, \mathcal{L}, \mathcal{W})$ as the weighted DAG $U\left(\mathcal{L}_{\mathcal{G}}\right)=\left(\mathcal{V} \cup\{\right.$ source, sink $\left.\}, E^{\prime}, \mathcal{W}_{E^{\prime}}\right)$, where

$E^{\prime}=\begin{gathered}\left\{\left(\text { source }, V^{\prime}\right): V^{\prime} \in \mathcal{V}\right\} \cup\left\{\left(V^{\prime}, \text { sink }\right): V^{\prime} \in \mathcal{V}\right\} \cup \\ \left\{\left(V^{\prime}, V^{\prime \prime}\right) \in \mathcal{V} \times \mathcal{V}: \forall v \forall v^{\prime}\left(v \in V^{\prime} \wedge v^{\prime} \in V^{\prime \prime} \rightarrow\left(v, v^{\prime}\right) \in E\right)\right\}\end{gathered}$,

For every (source, $\left.V^{\prime}\right) \in E^{\prime}$, we have

$\mathcal{W}^{\prime}\left(\right.$ source, $\left.V^{\prime}\right)=\sum_{v \in V \backslash V^{\prime}: \mathcal{L}(v) \leq \mathcal{L}\left(V^{\prime}\right)} \mathcal{W}(v)$,

For every $\left(V^{\prime}, \sin k\right) \in E^{\prime}$, we have

$\mathcal{W}^{\prime}\left(V^{\prime}, \sin k\right)=\sum_{v \in V \mathcal{L}^{\prime}\left(V^{\prime}\right)<\mathcal{L}(v)} \mathcal{W}(v)$,

And for every $\left(V^{\prime}, V^{\prime \prime}\right) \in E^{\prime}$ with $V^{\prime}, V^{\prime \prime} \in \mathcal{V}$ we have

$\mathcal{W}^{\prime}\left(V^{\prime}, V^{\prime \prime}\right)=\sum_{v \in V \backslash V^{\prime \prime}: \mathcal{L}^{\prime}\left(V^{\prime}\right)<\mathcal{L}(v) \mathcal{L}^{\prime}\left(V^{\prime \prime}\right)} \mathcal{W}(v)$.

For instance, the DAG in Fig. 19 is the result of unfolding the $w L-D A G$ in Fig. 11. The unfolding of a $w L-D A G$, in the worstcase scenario, is exponential in the size of $\mathcal{L}_{\mathcal{G}}$. Given a $w L$ $D A G \mathcal{L}_{\mathcal{G}}=(V, E, \mathcal{L}, \mathcal{W})$, we define $W_{\text {all }}\left(\mathcal{L}_{\mathcal{G}}\right)=\sum_{v \in V} \mathcal{W}(v)$ as the sum of all the weights associated with nodes in $V$. It is straightforward to prove that the union of all the internal nodes in a source-to-sink path in the unfolding of a $w L-D A G \mathcal{L}_{\mathcal{G}}$ is a thick path in $\mathcal{L}_{\mathcal{G}}$ and, on the other hand, every thick path in $\mathcal{L}_{\mathcal{G}}$ may be associated with a source-to-sink path in its unfolding. Moreover, for every source-to-sink path $p$ in the unfolding of $\mathcal{L}_{\mathcal{G}}$, let $w_{p}$ be its weight. The weight of the thick path associated with $p$ is exactly $W_{\text {all }}\left(\mathcal{L}_{\mathcal{G}}\right)-w_{p}$ (i.e., $\mathcal{L}_{\mathcal{G}}$ admits a thick path with value $\left.W_{\text {all }}\left(\mathcal{L}_{\mathcal{G}}\right)-w_{p}\right)$. With these premises, we can prove the following result.

Theorem 5 Given a $w L-D A G \mathcal{L}_{\mathcal{G}}=(V, E, \mathcal{L}, \mathcal{W})$, let $w$ the value of the shortest source-to-sink path in its unfolding. We have that the value of MAX-TP on $\mathcal{L}_{\mathcal{G}}=(V, E, \mathcal{L}, \mathcal{W})$ is equal to $W_{\text {all }}-w$.

Given a color-partial solution $C P S=\left(G_{\mathbf{r}}, \mathcal{C}^{+}, \mathcal{C}^{-}\right)$, procedure EdgeWise computes the value val $(C P S)$ (performed 
Fig. 19 The unfolding of the $w L-D A G$ of Figure 11 into a weighted DAG for solving the MAX-TP problem. The table below the graph provides the weights for source-to-node edges and node-to-sink edges, which are both represented by dashed lines. Continuous edges without labels have weight 0 . $P=\operatorname{source}\left\{v_{1}\right\}\left\{v_{2}, v_{3}\right\}\left\{v_{7}, v_{9}\right\}\left\{v_{8}\right\}\left\{v_{11}\right\}$ sink is a source-sink shortest path with value 4

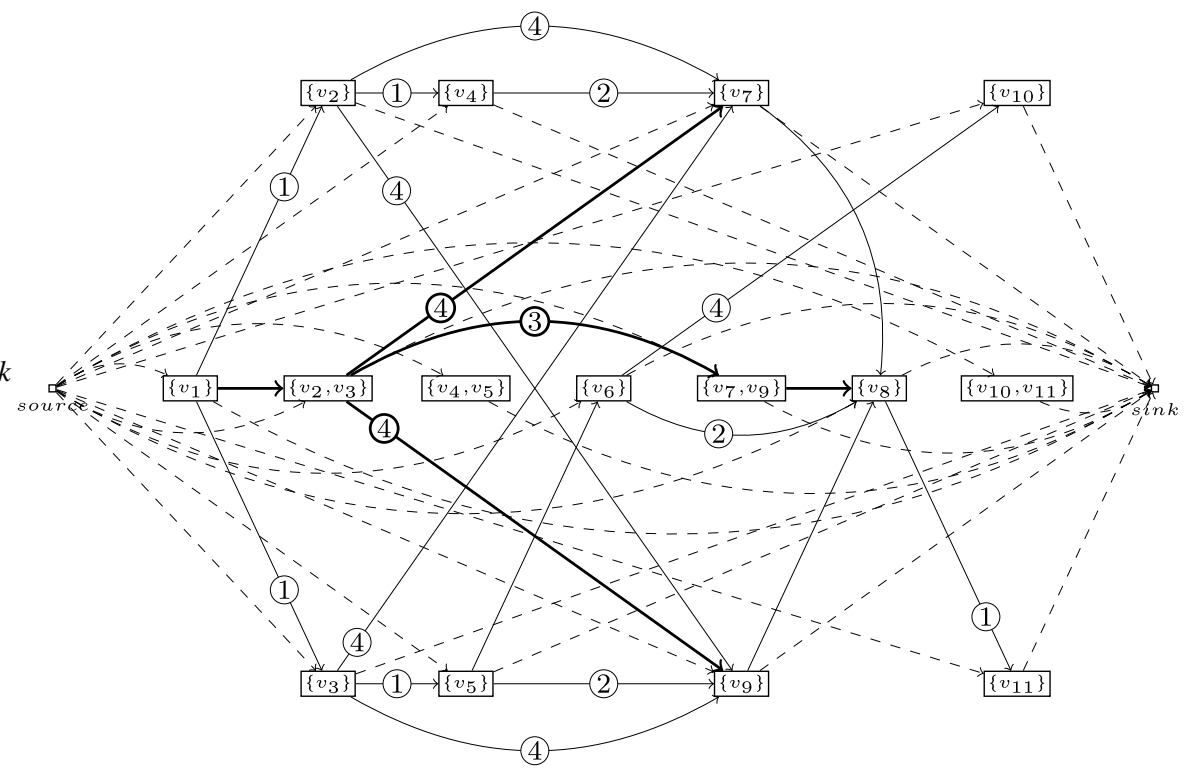

\begin{tabular}{|c|c|c|c|c|c|c|c|c|}
\hline$V^{\prime}$ & $\mathcal{W}^{\prime}\left(\right.$ source, $\left.V^{\prime}\right)$ & $\mathcal{W}^{\prime}\left(V^{\prime}\right.$, sink $)$ & $V^{\prime}$ & $\mathcal{W}^{\prime}\left(\right.$ source,$\left.V^{\prime}\right)$ & $\mathcal{W}^{\prime}\left(V^{\prime}\right.$, sink $)$ & $V^{\prime}$ & $\mathcal{W}^{\prime}\left(\right.$ source,$\left.V^{\prime}\right)$ & $\mathcal{W}^{\prime}\left(V^{\prime}\right.$, sink $)$ \\
\hline$\left\{v_{1}\right\}$ & 0 & 10 & $\left\{v_{6}\right\}$ & 5 & 5 & $\left\{v_{11}\right\}$ & 10 & 0 \\
$\left\{v_{2}\right\}$ & 2 & 8 & $\left\{v_{7}\right\}$ & 7 & 3 & $\left\{v_{2}, v_{3}\right\}$ & 1 & 8 \\
$\left\{v_{3}\right\}$ & 2 & 8 & $\left\{v_{8}\right\}$ & 8 & 2 & $\left\{v_{4}, v_{5}\right\}$ & 3 & 6 \\
$\left\{v_{4}\right\}$ & 4 & 6 & $\left\{v_{9}\right\}$ & 7 & 3 & $\left\{v_{7}, v_{9}\right\}$ & 6 & 3 \\
$\left\{v_{5}\right\}$ & 4 & 6 & $\left\{v_{10}\right\}$ & 10 & 0 & $\left\{v_{10}, v_{11}\right\}$ & 9 & 0 \\
\hline
\end{tabular}

by procedure PartialSolution in Fig. 18) summing up all the values $M_{C P S}^{j}$ for every $j \in J\left(G_{\mathbf{r}}\right)$. Each $M_{C P S}^{j}$ is computed as value of a source-to-sink shortest path (performed by procedure SourceSinkShortestPath in Figure 17) on the unfolding of $\mathcal{L}_{C P S}^{j}$ (built by procedure BuildDag in Fig. 17). For building $U\left(\mathcal{L}_{C P S}^{j}\right)$, on which we will compute value of a source-to-sink shortest path, we may need $\mathcal{O}\left(\operatorname{MaxSpace}\left(G_{\mathbf{r}}\right)^{2}\right)$ bits.

Finally, let us observe that procedure PartialSolution does not return only the value $\operatorname{val}(C P S)$ of the current color-partial solution $C P S=\left(G_{\mathbf{r}}, \mathcal{C}^{+}, \mathcal{C}^{-}\right)$. Since it effectively computes a minimal solution $\left(G_{\mathbf{r}}, G_{\mathbf{r}}^{+}, G_{\mathbf{r}}^{-}\right)$induced by CPS in order to provide $\operatorname{val}(C P S)$, it returns the set $\operatorname{colors}\left(G_{\mathbf{r}}, G_{\mathbf{r}} \backslash G_{\mathbf{r}}^{-}, G_{\mathbf{r}}^{-}\right)$, that is, the set of all and only the colors associated with active edges in $\left(G_{\mathbf{r}}, G_{\mathbf{r}} \backslash G_{\mathbf{r}}^{-}, G_{\mathbf{r}}^{-}\right)$. If such a set does not contain two colors $(x, y, z)$ and $\left(x, y, z^{\prime}\right)$ such that $z \neq z^{\prime}$, then we have that $\left(G_{\mathbf{r}}, \mathcal{C}^{+}, \mathcal{C}^{-}\right)$is a consistent solution and we may return val(CPS). Otherwise, procedure EdgeWiseMin takes a color $(x, y, z)$ in $\operatorname{colors}\left(G_{\mathbf{r}}, G_{\mathbf{r}} \backslash G_{\mathbf{r}}^{-}, G_{\mathbf{r}}^{-}\right)$such that there exists $\left(x, y, z^{\prime}\right)$ in $\operatorname{colors}\left(G_{\mathbf{r}}, G_{\mathbf{r}} \backslash G_{\mathbf{r}}^{-}, G_{\mathbf{r}}^{-}\right)$with $z \neq z^{\prime}$ and performs two recursive calls, one in which $\mathcal{C}^{+}$is updated to $\mathcal{C}^{+} \cup\{(x, y, z)\}$ and the other in which $\mathcal{C}^{-}$is updated to $\mathcal{C}^{-} \cup\{(x, y, z)\}$.

\section{Mining APE-FDs}

In this section, we consider the problem of mining $A P E$ -FDs on a given instance $\mathbf{r}$ of a temporal schema $R$ from a practical point of view. We will describe a prototype that performs such task. In particular, we point out two big computational challenges we addressed in the implementation of our prototype.

Let us start with the formal definition of our problem. Given a temporal schema $R=U \cup\{V T\}$, an instance $\mathbf{r}$ of $R$, a nonempty set $J \subseteq U$, a threshold $0 \leq \epsilon \leq 1$, and a value $k \in \mathbb{N}$, we denote as $\mathcal{P E}(\mathbf{r}, k, \epsilon)$ the set of all the $A P E$-FDs $\left[\Delta_{k}\left(\tau_{J}^{R}\right)\right] X \bar{Y} \stackrel{\epsilon}{\rightarrow} \bar{Z}$, formally introduced in "Discovering Pure Temporally Evolving Functional Dependencies" section, such that $\mathbf{r} \vDash\left[\Delta_{k}\left(\tau_{J}^{R}\right)\right] X \bar{Y} \stackrel{\epsilon}{\rightarrow} \bar{Z}$.

A set $\mathcal{S}$ of $A P E$-FDs is complete if and only if for every $\left[\Delta_{k}\left(\tau_{J}^{R}\right)\right] X \bar{Y} \stackrel{\epsilon}{\rightarrow} \bar{Z}$ belonging to $\mathcal{S}$, there exists $X^{\prime} \subseteq X$ and $\bar{Y}^{\prime} \subseteq \bar{Y}$ such that $\left[\Delta_{k}\left(\tau_{J}^{R}\right)\right] X^{\prime} \bar{Y}^{\prime} \stackrel{\epsilon}{\rightarrow} \bar{Z} \in \mathcal{P E}(\mathbf{r}, k, \epsilon)$. A complete $A P E$-FD-set $\mathcal{P E}(\mathbf{r}, k, \epsilon)$ is minimal if and only if for every $\left[\Delta_{k}\left(\tau_{J}^{R}\right)\right] X \bar{Y} \stackrel{\epsilon}{\rightarrow} \bar{Z} \in \mathcal{S}$, set $\mathcal{S} \backslash\left\{\left[\Delta_{k}\left(\tau_{J}^{R}\right)\right] X \bar{Y} \stackrel{\epsilon}{\rightarrow} \bar{Z}\right\}$ is not complete anymore. Given a complete minimal $A P E$-FD-set $\mathcal{P E}(\mathbf{r}, k, \epsilon)$, every subset $\overline{\mathcal{P E}}(\mathbf{r}, k, \epsilon) \subseteq \mathcal{P E}(\mathbf{r}, k, \epsilon)$ is called a minimal partial.

Given a temporal schema $R=U \cup\{V T\}$, an instance $\mathbf{r}$ of $R$, a nonempty set $J \subseteq U$, a threshold $0 \leq \epsilon \leq 1$ and a value $k \in \mathbb{N}$, we are interested in finding a minimal complete set 
$\mathcal{P E}(\mathbf{r}, k, \epsilon)$. However, in order to do that, we have to deal with two computational problems:

- the smallest minimal complete set $\mathcal{S}$ may be exponential in the size of $U$ with respect to some given temporal schemata $R=U \cup\{V T\}$, instances $\mathbf{r}$ of $R$, nonempty sets $J \subseteq U$, thresholds $0 \leq \epsilon \leq 1$, and values $k \in \mathbb{N}$;

- given a single $A P E-\mathrm{FD}\left[\Delta_{k}\left(\tau_{J}^{R}\right)\right] X \bar{Y} \stackrel{\epsilon}{\rightarrow} \bar{Z}$ on a schema $R=U \cup\{V T\}$, and an instance $\mathbf{r}$ of $R$, deciding whether or not $\mathbf{r} \vDash\left[\Delta_{k}\left(\tau_{J}^{R}\right)\right] X \bar{Y} \stackrel{\epsilon}{\rightarrow} \bar{Z}$ is a NP-complete problem.

The first result may be derived by leveraging a result of Kivinen et al. [16] on approximate functional dependencies. The second result is proved in "The Computational Complexity of Checking APE-FD" section. However, such theoretical bounds are both difficult to achieve in real-world domains. For instance, the size of $\mathcal{P E}(\mathbf{r}, k, \epsilon)$ could be exponential in $|U|$, but in real-case scenarios, we have that $|U|$ is often less than 50/60 elements. Moreover, the instance built in [16] for achieving the exponential lower bound does not occur in real-world instances. The complexity of checking a single $A P E$-FD is even worse. Indeed, checking a single $A P E$-FD is NP-Complete in the number of tuples, which may be very high and increasing time after time. This problem is known as the curse of cardinality, and its relevance has been recently considered for temporal inference of sequential patterns in [18]. Even in this case, the instance specified in "The Computational Complexity of Checking $A P E$-FD" section for proving the NP-hardness result has been built in a very complex and constrained way. Such an instance does not even remotely resemble some real-world scenario. Thus, we are allowed to design and implement a prototype for the practical mining of such dependencies on real-world datasets and evaluate its performances.

\section{Prototype Overview}

Even though the results reported in "The Computational Complexity of Checking APE-FD" section are not completely encouraging and according to the last comments in the previous section, we developed a prototype. Given a temporal schema $R=U \cup\{V T\}$, an instance $\mathbf{r}$ of $R$, a nonempty set $J \subseteq U$, a threshold $0 \leq \epsilon \leq 1$ and a value $k \in \mathbb{N}$, it returns a minimal complete set $\mathcal{P E}(\mathbf{r}, k, \epsilon)$. The prototype is named Attila(Approximate Temporal Tailored Inference Lean Application). In the following, after providing a high-level description of Attilamodules and their interaction, we focus with a detailed description on novel ideas underlying the design of the prototype. Attilawas implemented according to the principles of distributed programming. Different tasks are executed by different processes (possibly executed on different machines). Attila is composed by three main processes:
- Worker is responsible for maintaining a representation of the minimal partial $\overline{\mathcal{P E}}(\mathbf{r}, k, \epsilon)$ of $\mathcal{P} \mathcal{E}(\mathbf{r}, k, \epsilon)$. Thus, Worker maintains a compact representation both of the set of the APE-FDs already checked and of the APE-FDs that remain to be checked. It updates its state according to the last $A P E$-FD $\left[\Delta_{k}\left(\tau_{J}^{R}\right)\right] X \bar{Y} \stackrel{\epsilon}{\rightarrow} \bar{Z}$ that has been checked. The next state depends on the fact that either $\mathbf{r} \vDash\left[\Delta_{k}\left(\tau_{J}^{R}\right)\right] X \bar{Y} \stackrel{\epsilon}{\rightarrow} \bar{Z}$ or $\mathbf{r} \backslash \vDash\left[\Delta_{k}\left(\tau_{\epsilon}^{R}\right)\right] X \bar{Y} \stackrel{\epsilon}{\rightarrow} \bar{Z}$. In both cases, Worker marks $\left[\Delta_{k}\left(\tau_{J}^{R}\right)\right] X \bar{Y} \stackrel{\epsilon}{\rightarrow} \bar{Z}$ to not be checked anymore. In this way, it can determine precisely the next APE-FD to check;

- Contributor has to check a single APE-FD against the instance r.It retrieves $A P E$-FD from Worker. When Contributor gets an $A P E-\operatorname{FD}\left[\Delta_{k}\left(\tau_{J}^{R}\right)\right] X \bar{Y} \stackrel{\epsilon}{\rightarrow} \bar{Z}$, it schedules jobs among the computational units that will effectively check the given $A P E-\mathrm{FD}$;

- Sub-Contributor is the basic computational unit and depends on Contributor. Sub-Contributor leverages the graph representation generated by Contributor for resolving a subproblem of the original one. More precisely, it receives a subproblem of the one stored by Contributor for checking a given $\left[\Delta_{k}\left(\tau_{J}^{R}\right)\right] X \bar{Y} \stackrel{\epsilon}{\rightarrow} \bar{Z}$ against it. During the computation, Contributor may send a reduction of the subproblem Sub-Contributor is dealing with. Indeed, Contributor may assign portions of a problem to several Sub-Contributors.

Processes composing $\mathcal{A t t i l a a r e}$ hierarchically organized. A Worker could manage minimal partial $\overline{\mathcal{P E}}(\mathbf{r}, k, \epsilon)$, but more Contributors are needed for checking multiple dependencies at the time. Each Contributor may have several SubContributors in order to speed up the checking procedure.

Now, let us consider in some detail each process type, in order to give a general idea of how computations are handled. Worker has to manage the minimal partial $\overline{\mathcal{P E}}(\mathbf{r}, k, \epsilon)$. At the end of computation, $\overline{\mathcal{P E}}(\mathbf{r}, k, \epsilon)$ turns out to be a minimal complete set, according to the goal of our distributed procedure. Worker interacts only with its pool of Contributors. Figure 20 depicts how such interaction happens, by a BPMN choreography [22]. A Contributor can register to the Worker at any time incrementing by one the number of $A P E$-FDs that may be checked simultaneously. Worker may receive $A P E$-FD request only by registered Contributors. To this regard, it keeps two auxiliary APE-FD-sets Pending and Assigned $\subseteq$ Pending. Pending is a minimal set of $A P E$-FDs such that Pending $\cap \overline{\mathcal{P E}}(\mathbf{r}, k, \epsilon)=\emptyset$ and, if for every $\left[\Delta_{k}\left(\tau_{I}^{R}\right)\right] X \bar{Y} \stackrel{\epsilon}{\rightarrow} \bar{Z} \in$ Pending $\mathbf{r} \vDash\left[\Delta_{k}\left(\tau_{J}^{R}\right)\right] X \bar{Y} \stackrel{\epsilon}{\rightarrow} \bar{Z}$, then Pending $\cup \overline{\mathcal{P E}}(\mathbf{r}, k, \epsilon)$ is a minimal complete $A P E$ -FD-set. Pending contains all APE-FDs among whom Worker must choose every time it is asked for an APE-FD by some of its Contributors. Assigned represents all APE -FDs in Pending already assigned to Contributors. Every time a Contributor returns the result of $A P E-F D$-check 
Fig. 20 A BPMN choreography showing the interaction between a Worker and its (possibly) many Contributors

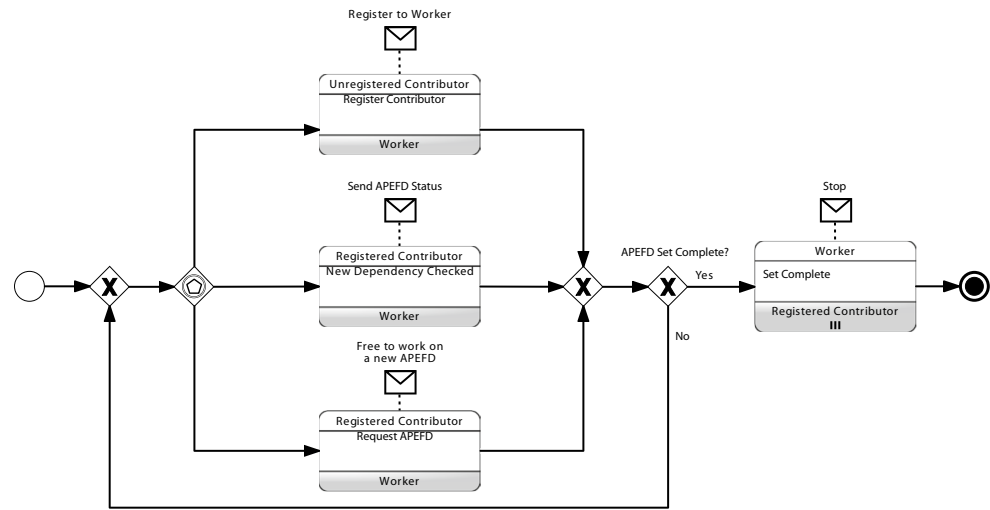

on an element of Assigned, Pending and $\overline{\mathcal{P E}}(\mathbf{r}, k, \epsilon)$ are updated according to the fact that either $\mathbf{r} \vDash\left[\Delta_{k}\left(\tau_{J}^{R}\right)\right] X \bar{Y} \stackrel{\epsilon}{\rightarrow} \bar{Z}$ or $\mathbf{r} \backslash \vDash\left[\Delta_{k}\left(\tau_{J}^{R}\right)\right] X \bar{Y} \stackrel{\epsilon}{\rightarrow} \bar{Z}$. More precisely, we have that $\left[\Delta_{k}\left(\tau_{J}^{R}\right)\right] X \bar{Y} \stackrel{\leftrightarrow}{\rightarrow} \vec{Z}$ is removed from both Pending and Assigned, and

- if $\mathbf{r} \vDash\left[\Delta_{k}\left(\tau_{J}^{R}\right)\right] X \bar{Y} \stackrel{\epsilon}{\rightarrow} \bar{Z}$, then Worker inserts such APE-FD in $\overline{\mathcal{P E}}(\mathbf{r}, k, \epsilon)$;

- if $\mathbf{r} \backslash \vDash\left[\Delta_{k}\left(\tau_{J}^{R}\right)\right] X \bar{Y} \stackrel{\epsilon}{\rightarrow} \bar{Z}$, then for each attribute $W \in U \backslash(X \cup Y \cup\{Z\})$, dependencies $\left[\Delta_{k}\left(\tau_{J}^{R}\right)\right] X W \bar{Y} \stackrel{\epsilon}{\rightarrow} \bar{Z}$ and $X \overline{Y W} \stackrel{\epsilon}{\rightarrow} \bar{Z}$ are inserted in Pending.

The main operations Worker performs are:

1. (i) it pulls an $A P E-F D\left[\Delta_{k}\left(\tau_{J}^{R}\right)\right] X \bar{Y} \stackrel{\epsilon}{\rightarrow} \bar{Z}$ out of Pending,

2. it updates $\overline{\mathcal{P E}}(\mathbf{r}, k, \epsilon)$ if needed, and

3. (iii) it updates Pending and Assigned as soon as the status of a new dependency is discovered.

We use Ordered Binary Decision Diagrams (OBDDs) [1] as data structures allowing an efficient execution of such operations. An OBDD is a single-rooted directed acyclic graph that represents a propositional formula $\psi$. A propositional variable is associated with every node as a label, except for the only terminal node $1 .^{2}$ Any nonterminal node $v$ may have at most two outgoing edges low $(v)$ (dotted line, depicted in Fig. 21) and $h i g h(v)$ (solid lines, depicted in Fig. 21). low (v) $(h i g h(v))$ means that variable $v$ is taken with value $0(1)$. A variable truth assignment is represented by a path from the root to terminal node 1 . Thus, an OBDD represents the set of all truth assignments for a given formula $\psi$. Worker uses three different OBDDs corresponding to formulas $\psi_{\overline{\mathcal{P E}}}, \psi_{P}$ and $\psi_{A}$, to keep track of sets $\overline{\mathcal{P} \mathcal{E}}(\mathbf{r}, k, \epsilon)$, Pending and Assigned, respectively. APE-FDs in $\overline{\mathcal{P E}}(\mathbf{r}, k, \epsilon)$ correspond to all and only the solutions of formula $\psi_{\overline{\mathcal{P E}}}$ (and the same for

\footnotetext{
${ }^{2}$ We use OBDD without the 0 terminal node.
}

$A P E$-FDs in Pending with respect to formula $\psi_{P}$ and for $A P E$ -FDs in Assigned with respect to $\psi_{A}$ ). Hereinafter, we will use $\psi$ for denoting both the formula and the OBDD corresponding to all its possible solutions. Informally, updates of these three sets are implemented by adding conjuncts/disjuncts to their respective formulas.

For representing set $\overline{\mathcal{P E}}(\mathbf{r}, k, \epsilon)$ as all and only the solutions of a formula, it suffices to assign to each attribute $X_{i} \in U$ three variables $x_{i}, \bar{x}_{i}, \vec{x}_{i}$. Then, formula $\psi \overline{\mathcal{P E}}$ has be satisfied by assignments $\sigma:\left\{x_{1}, \ldots, x_{n}, \bar{x}_{1}, \ldots\right.$, $\left.\bar{x}_{n}, \vec{x}_{i}, \ldots, \vec{x}_{n}\right\} \rightarrow\{0,1\}$. An APE-FD $\left[\Delta_{k}\left(\tau_{J}^{R}\right)\right] X \bar{Y} \stackrel{\epsilon}{\rightarrow} \bar{Z}$ belongs to $\overline{\mathcal{P}}(\mathbf{r}, k, \epsilon)$ if and only if the assignment $\sigma\left(x_{i}\right)=1 \forall X_{i} \in X$, $\sigma\left(\bar{y}_{j}\right)=1 \forall \bar{Y}_{j} \in \bar{Y}$, and $\sigma(\vec{z})=1$ satisfies $\psi_{\overline{\mathcal{P}} \mathcal{E}}$. According to this approach, for instance, $a_{1} \wedge a_{2} \wedge \bar{a}_{3} \wedge \vec{a}_{4}$ represents $A P E$ $\operatorname{FD}\left[\Delta_{k}\left(\tau_{J}^{R}\right)\right] A_{1} A_{2} \bar{A}_{3} \rightarrow \bar{A}_{4}$. Clearly, if a formula $\psi_{\overline{\mathcal{P E}}}$ represents all the possible $A P E$-FDs, an OBDD for $\psi_{\overline{\mathcal{P E}}}$ represents them as well. The same approach may be used for sets Pending and Assigned. Hereinafter, we refer to $\psi$ as the OBDD representing all and only the assignments $\sigma$ such that $\sigma \vDash \psi$.

A Worker begins the APE-FD mining task by initializing two OBBDs representing $\psi_{\overline{\overline{P E}}}=\psi_{A}=\perp$. Initially, $\psi_{\overline{\overline{P E}}}=\perp$ (i.e., $\overline{\mathcal{P E}}(\mathbf{r}, k, \epsilon)=\emptyset$ ) since the distributed procedure has not discovered any valid $A P E$-FD yet. $\psi_{P}$ is true only for those assignments that represent well-formed $A P E$-FDs. In order to extract from $\psi_{P}$ an $A P E$-FD to be tested, we take the solution associated with any root-to-terminal path in the OBDD for $\psi_{P} \wedge \neg \psi_{A}$. For inserting an APE-FD $\left[\Delta_{k}\left(\tau_{J}^{R}\right)\right] X \bar{Y} \stackrel{\epsilon}{\rightarrow} \bar{Z}$

Fig. 21 The update of the set $\overline{\mathcal{P E}}(\mathbf{r}, k, \epsilon)=\left\{\left[\Delta_{k}\left(\tau_{\text {Patld }}^{\text {Cont }}\right)\right] G A F\right.$, $P h y s \stackrel{\epsilon}{\rightarrow} \overline{C T}$ (left) into the set $\overrightarrow{\mathcal{P E}}(\mathbf{r}, k, \epsilon)=\left\{\left[\Delta_{k}\left(\tau_{\text {Pattl }}^{\text {Contact }}\right)\right] G A F\right.$, Phys $\left.\stackrel{\epsilon}{\rightarrow} \overline{C T},\left[\Delta_{k}\left(\tau_{\text {Pattd }}^{\text {Cont }}\right)\right] G A F, \overline{\text { Phys }} \stackrel{\bullet}{\rightarrow} \overline{C T}\right\}$ (right)
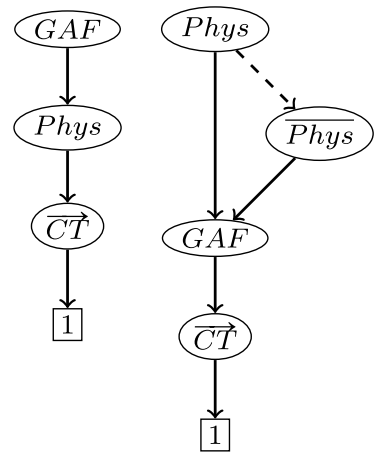
in $\overline{\mathcal{P E}}(\mathbf{r}, k, \epsilon)$, it suffices to update $\psi_{\overline{\mathcal{P E}}}$ to $\psi_{\overline{\mathcal{P E}}} \vee \psi$ where $\psi=\bigwedge_{X_{i} \in X} x_{i} \wedge \bigwedge_{X_{i} \in Y} \bar{x}_{i} \wedge \vec{z}$. Moreover, for deleting a solution from Pending it suffices to update $\psi_{P}$ :

- if we have that $\mathbf{r} \vDash\left[\Delta_{k}\left(\tau_{J}^{R}\right)\right] X \bar{Y} \stackrel{\epsilon}{\rightarrow} \bar{Z}$, then we update $\psi_{P}$ to $\psi_{P} \wedge \neg \psi$

- if we have that $\mathbf{r} \backslash \vDash\left[\Delta_{k}\left(\tau_{J}^{R}\right)\right] X \bar{Y} \stackrel{\epsilon}{\rightarrow} \bar{Z}$, then we update $\psi_{P}$ to $\psi_{P} \wedge \bar{\psi}$ where $\bar{\psi}=\vec{z} \rightarrow\left(\bigvee_{X_{i} \in U \backslash X} x_{i} \vee \bigvee_{X_{i} \in U \backslash Y} \bar{x}_{i}\right)$ (i.e., if $\vec{z}$ holds, then at least one of variables not contained in the formula for the given $A P E$-FD must hold).

It is worth noting that we have a search operation for $A P E$ -FDs that is linear in $\mid U l$. Moreover, Boolean operations on OBDDs are implemented in very efficient way by many packages on the market (in our prototype, we used BuDDy [17]). This solution allows us to have a compact representation of sets of $A P E$-FDs that can be manipulated efficiently.

Figure 21 shows an example of how $\overline{\mathcal{P E}}(\mathbf{r}, k, \epsilon)$ is updated if we represent it through formula $\psi_{\overline{\mathcal{P E}}}$. In this example, we borrow two dependencies from the psychiatric case register introduced in a simplified way in "Discovering Pure Temporally Evolving Functional Dependencies" section and discussed in detail in "Mining APE-FDs on Clinical Domains" section. The real-world schema differs from the example since patients are identified by PatId attribute in place of their names. Furthermore, several attributes are used for storing information regarding registered calls. The most significant attribute is Global Assessment of Functioning $(G A F)$ : it is a numeric value provided by the physician at the end of the call, and it scores the patient's mental health status. Figure 21 shows an update operation on $\overline{\mathcal{P E}}(\mathbf{r}, k, \epsilon)=\left\{\left[\Delta_{k}\left(\tau_{\text {Patld }}^{\text {Contact }}\right)\right] G A F\right.$, Phys $\left.\underset{\mathcal{P E}}{\rightarrow} \overline{C T}\right\}$, where $\overline{\mathcal{P E}}(\mathbf{r}, k, \epsilon)$ is updated to $\overline{\mathcal{P E}}^{\prime}(\mathbf{r}, k, \epsilon)$ $=\left\{\left[\Delta_{k}\left(\tau_{\text {Pattd }}^{\text {Contact }}\right)\right] G A F\right.$, Phys $\left.\stackrel{\epsilon}{\rightarrow} \overline{C T},\left[\Delta_{k}\left(\tau_{\text {Patld }}^{\text {Contact }}\right)\right] G A F, \overline{\text { Phys }} \stackrel{\epsilon}{\rightarrow} \overline{C T}\right\}$. As one may notice, each node $v$ has at most two outgoing edges, one solid and one dashed representing high(v) and $l o w(v)$ respectively. Taking a solid edge high(v) in a root-toterminal path denotes that the attribute corresponding to $v$ belongs to the dependency. Taking a dashed edge low $(v)$ in a root-to-terminal path denotes that the attribute corresponding to $v$ does not belong to the dependency. As an example, the OBDD shown in Fig. 21 (right) features two paths from the root node, labeled Phys, to terminal node 1. Since the edge (Phys, $\overline{\text { Phys }}$ ) is dashed, the path Phys, $\overline{P h y s}, G A F, \overrightarrow{C T}, 1$ represents the dependency $\left[\Delta_{+\infty}\left(\tau_{\text {Patld }}^{\text {Cont }}\right)\right] G A F, \overline{\text { Phys }} \rightarrow \overline{C T}$. Such dashed edge implies that attribute Phys is not taken in $X$ while it is taken in $Y$ because the outgoing edge the path takes from $\overline{P h y s}$ is a continuous one.

Let us consider now the Contributor process. Until now we just considered it as a process which asks Worker for a dependency and eventually answers whether $\mathbf{r} \vDash\left[\Delta_{k}\left(\tau_{J}^{R}\right)\right] X \bar{Y} \stackrel{\leftrightarrow}{\rightarrow} \bar{Z}$ holds or not, as shown by the BPMN choreography in Fig. 20. Thus, Contributor is responsible for checking a single $A P E-F D$ at a time. Since the complexity is intractable (recall that the problem in NP-Complete), Contributor does not deal directly with the computation. Indeed, as mentioned before, it splits a problem among several computational units called Sub-Contributor $s$.

The way in which a Contributor deals with its pool of Sub-Contributors is very close to the interaction between Worker and its Contributors, and it is described by the BPMN choreography diagram provided in Fig. 22. The status of the problem is managed by Contributor, and it is represented by a binary tree, where each node is labeled with a tuple and its two children represent either the case in which the tuple is inserted in the current solution or it is removed from it. Subproblems are generated by asserting that a tuple belongs or not to the final solution. This procedure generates a tree. Initially, the whole tree is given to a single Sub-Contributor to visit. Suppose that a new SubContributor registers himself to the same Contributor during a computation. Such Contributor selects the subproblem of a Sub-Contributor and a tuple $t$ in $\mathbf{r}$. Contributor splits the subproblem into two parts, one in which $t$ must belong to the solution and the other in which $t$ does not belong to the solution. One portion is given to the new Sub-Contributor and the "old" Sub-Contributor is notified to reduce its problem to the other portion. Usually, we have multiple Sub-Contributors that work in a subtree rooted at the node where the reduce operation happens, and thus, as reported in Fig. 22, we have to notify all of them about the reduction.

Figure 23 shows an example of how Contributor works. Suppose that there is exactly one Sub-Contributor $s c_{1}$ that is exploring the tree (a) on the top of Fig. 23. At a certain point, a new Sub-Contributor $s c_{2}$ registers himself to the Contributor and requests a subproblem. Now Contributor looks at the active subproblem and chooses the one of $s c_{1}$. At the root of such problem, there is tuple $t_{1}$. Therefore, Contributor splits the subproblem into two more subproblems: one where $t_{1}$ is forced to be deleted (tree (b) in Fig. 23), and the other where $t_{1}$ is kept (tree (c) in Fig. 23). Finally, the exploration of subtree (c) is given to $s c_{2}$ and $s c_{1}$ is notified that its exploration of the tree (a) is reduced to the exploration of the subtree (b).

Sub-Contributor is the minimal computation unit: it simply performs tasks assigned by its master Contributor. SubContributor listens constantly to its Contributor in order to receive reduction of its current subproblem for speeding up the process; meanwhile, it explores its current subproblem searching for a solution. Sub-Contributor operates in two symmetric ways that may be seen as two concurrent threads. The first thread assumes that its subproblem contains the solution and performs a depth-first search of the tree in order to find it. The other thread assumes that the subproblem does not contain the solution and tries to find a counterexample. 


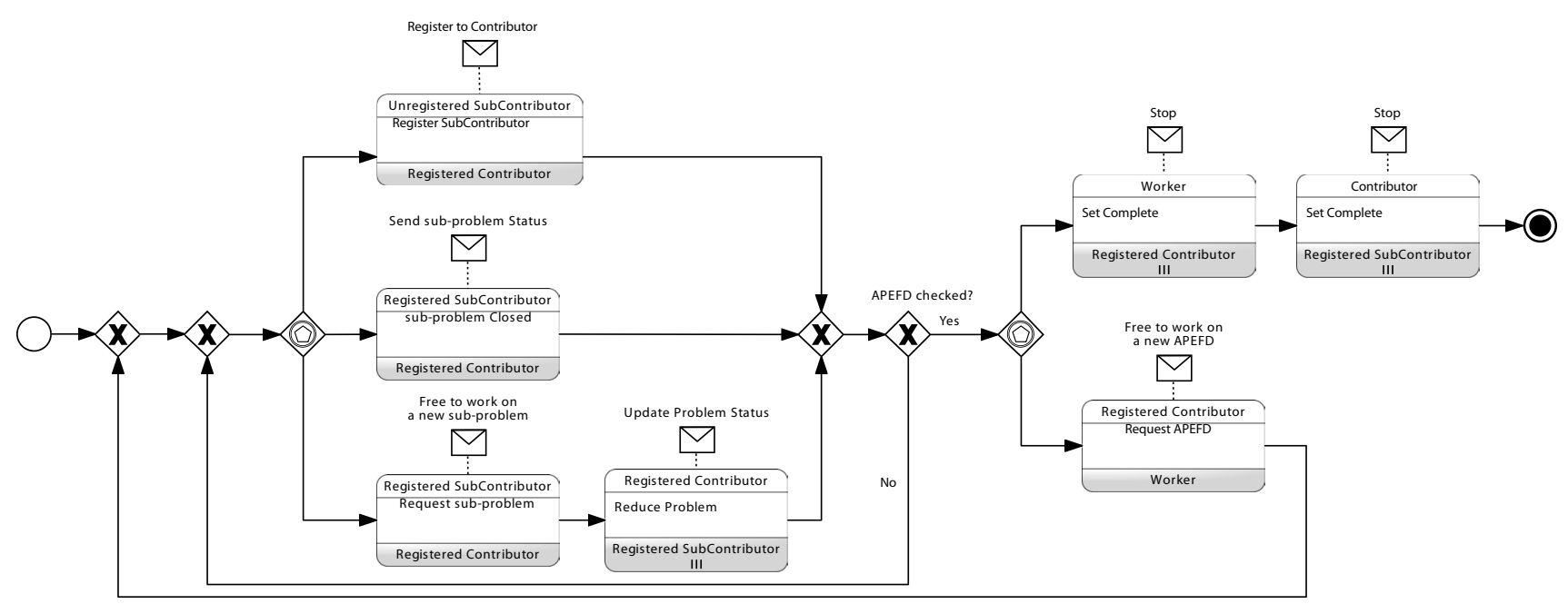

Fig. 22 A BPMN choreography showing the interaction between a Contributor and its (possibly) many Sub-Contributors

(a)

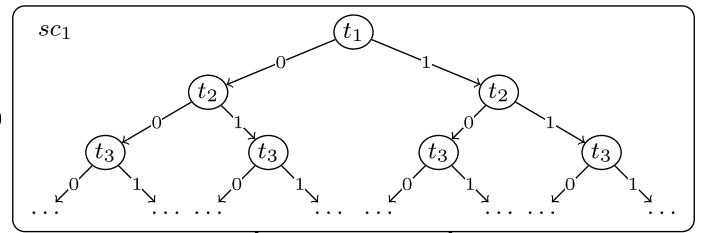

(b)
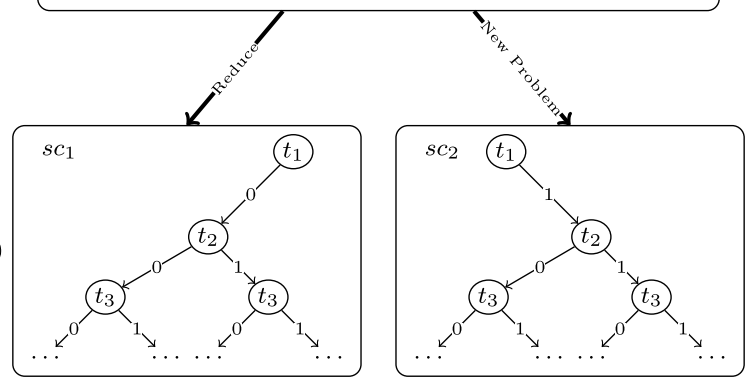

Fig. 23 A graphical account of how the tree is split among Sub-Contributors

In order to deal with the latter task, the Sub-Contributor translates the subproblem into a linear programming problem and verifies its feasibility. This symmetric approach turns out to be very efficient. Attilamakes use of the open-source linear programming library GNU Linear Programming Kit (GLPK) [24] to perform such linear programming tasks.

\section{Mining APE-FDs on Clinical Domains}

In this section, we discuss APE-FDs obtained through Attila. These results may be also considered as an early validation of our prototype. As we already mentioned, we focused on two different clinical domains. The first one is that of psychiatry. In this domain, one of the main sources of information consists of data acquired during the (mainly, telephonic) contacts between patients and psychiatrists. "Discovering Pure Temporally Evolving Functional Dependencies" section provides a detailed description of this domain. Attila allowed us to extract the following APE-FDs from relation Contact:

- $\left[\Delta_{+\infty}\left(\tau_{\text {Patld }}^{\text {Contact }}\right)\right] G A F \stackrel{\epsilon}{\rightarrow} \overline{\text { numPsychologists }}$ with $\epsilon=0.1$. This dependency represents the fact that, considering two consecutive calls of the same patient, the number of psychologists involved in the second call uniquely depends on the GAF score of the patient during the first call. It may highlight that some (maybe implicit) policy determines the number of psychologists required for a contact, according to the conditions the given patient showed in the previous call.

- $\left[\Delta_{+\infty}\left(\tau_{\text {Patld }}^{\text {Contact }}\right)\right]$ Service, $G A F \stackrel{\epsilon}{\rightarrow} \overline{C T}$ with $\epsilon=0.1$. Informally, it means that for each pair of consecutive calls for the same patient, the previous patient's GAF score and Service (clinical psychiatry, medical psychology, psychotherapy, ...) uniquely determines the next contact type (family member, a neighbor, the police, ...).

- $\left[\Delta_{+\infty}\left(\tau_{\text {Patld }}^{\text {Contact }}\right)\right] G A F$, Physician $\rightarrow \overline{\text { Request }}$ with $\epsilon=0.1$. This dependency says that the next actions on patients are mainly based on the previous GAF score and physician. For each pair of consecutive calls, the request (it could be group psychotherapy, family psychotherapy, legal medical evaluation, ...) decided during the second call depends on the physician and on the GAF score of the given patient during the first call.

The second clinical domain is that of pharmacovigilance, which is the science related to the management and prevention of suspected adverse reactions induced by drugs [34]. Premarketing trials are not able to discover all adverse reactions induced by the investigated drug. This is due to trial 
limitations, e.g., short timespan of the study, highly selected test groups, and so on. Adverse drug reactions (ADRs) may, indeed, go undetected and become evident when the drug is already on the market [28]. Therefore, marketed drugs require a continuous monitoring of their possible effects. The spontaneous reporting of ADRs allows healthcare stakeholders to identify unexpected reactions and to inform regulating authorities about them. This practice is extremely important, provides early warnings and requires limited economic and organizational efforts and resources [21]. Among its multiple advantages, spontaneous reporting allows one to consider every drug on the market and any category of patients. It investigates possible relationships between one or more adverse reactions and one or more drugs. physicians, chemists or citizens are allowed to submit reports. The analysis focuses on unknown or completely undocumented relationships and may suggest a potential cause-effect link between ADRs and drugs, classified as "suspected" or "concomitant." Any report contains both demographic and specific pharmacovigilance data, as patient information (age, nationality, gender, weight, outcome of reactions, and so on), drug(s) involved in the suspected reaction(s) (identified by their Anatomical Therapeutic Chemical - ATC - classification, brand name, dosage), and the description of the occurred adverse reaction(s) encoded by means of the MedDRA classification [20], the entry date, the period of the adverse reaction and the periods of drug administrations. These temporal data are then processed and analyzed to possibly discover any cause-effect relationship among drugs and reaction(s) in different time periods, or according to the exposure timespan. In this case, we consider the evolution of reports for the same drug (by using PhProd (Pharmaceutical Product, i.e., active principle) for performing the join in the evolution expression). This way, we may observe whether the therapy decided by physicians is influenced by previous adverse reactions. As an example, the fact that physicians are aware of past cases of adverse events could determine changes in drug dosages. Changing the prescribed drug quantities for patients because of previously suspected drugs could be considered as attempt of avoiding such adverse reactions.

Among APE-FDs extracted from a recent instance of Reports schema of the Italian Network of Pharmacovigilance, we introduce here the following $A P E-F D$.

- $\left[\Delta_{+\infty}\left(\tau_{\text {PhProd }}^{\text {Reports }}\right)\right]$ PhProd,Dos $\stackrel{\epsilon}{\rightarrow} \overline{\text { Dos }}$ with $\epsilon=0.2$. Such a dependency may highlight that, when an ADR is reported, the dose is usually adjusted in the same way for most patients, depending on the previous administrations. Such $A P E-F D$ may suggest that Italian physicians methodically consider the Italian Network of Pharmacovigilance in managing drug therapies.

\section{Performance Analysis}

In this section, we present a short and preliminary performance analysis of Attila and of its components. We executed two kinds of test. The first test was done using a single machine. This way, we obtained a first evaluation of the time required for mining multiple $A P E$-FDs on a large real-world database. The second test focused on a single $A P E-\mathrm{FD}$, but considering some distributed architecture, using a server and at most two distinct remote machines. This test allows us to observe whether the time required for checking a single $A P E$ -FD decreases, when the problem is distributed among different computational units.

We started by analyzing the performances of the whole system, when the computation is entirely done by a single machine. We tested Attila on an instance of schema Contact, consisting of approximately $1.5 \cdot 10^{6}$ rows. APE-FDs as $\left[\Delta_{+\infty}\left(\tau_{\text {Pattd }}^{\text {Contact }}\right)\right] X \bar{Y} \rightarrow \bar{Z}$ were extracted, with a threshold $\epsilon=0.1$. Figure 24 depicts the result of this first experiment. Attilaverified almost 4500 APE-FDs in about 10 days. Figure 24 shows through a pie chart that checked $A P E$-FDs (i.e., holding and not holding) are less than half of possible $A P E$-FDs. Indeed, many APE-FDs denoted as superset are subsumed by the checked and holding APE-FDs. Thus, they have not been tested (i.e., only minimal APE-FDs have been tested). Moreover, Worker is often idling, as Contributors perform most of the computation. As described in "Mining $A P E$-FDs" section, a Contributor has to visit a tree, which is exponentially large w.r.t. the instance size. Even though this operation is theoretically unfeasible for large instances, by employing simple pruning conditions (as, for example, too many tuples deleted, existence of violated constraints, and so on), the tree size may be reduced.

Finally, we analyzed the interactions between Contributor and Sub-Contributors by checking APE-FD $\left[\Delta_{+\infty}\left(\tau_{\text {PhProd }}^{\text {Repors }}\right)\right]$ PhProd, Dos $\stackrel{\epsilon}{\rightarrow} \overline{\text { Dos }}$ with $\epsilon=0.2$, discussed in "Mining APE-FDs on Clinical Domains" section and related to the pharmacovigilance domain. Figure 25a depicts some comparisons between various configurations of Sub-Contributors when checking the given dependency. We considered five possible situations:

(1) a single local Sub-Contributor(Server), running on the same machine where Contributor is running;

(2) a single local Sub-Contributor, and a remote SubContributor(Remote);

(3) two local Sub-Contributors and a single remote SubContributor,

(4) two remote Sub-Contributors, i.e., two separate physical machines with identical hardware/specs running a Sub-Contributor each;

(5) a single local Sub-Contributor, and two remote SubContributors. 
Fig. 24 Result of the execution of Attilaon a single machine (Intel Core i3(TM) CPU M 330 $2.13 \mathrm{GHz}, 4 \mathrm{~GB})$ on an instance of table Contact $\left(\sim 1.5 \cdot 10^{6}\right.$ rows)
Dependencies Tested: 4427

Execution Time (hours): 218

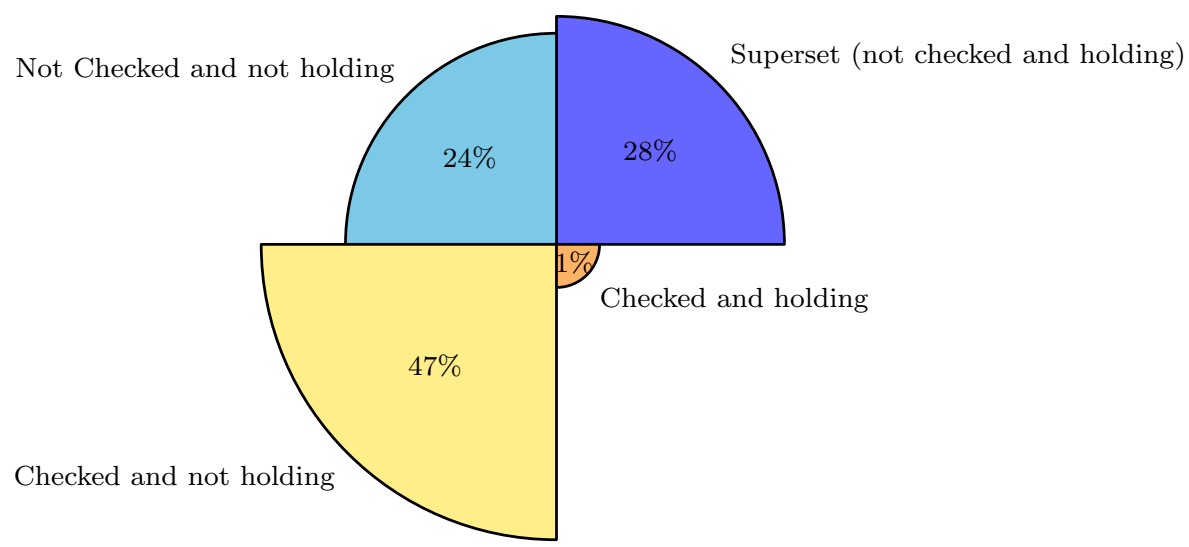

(a)

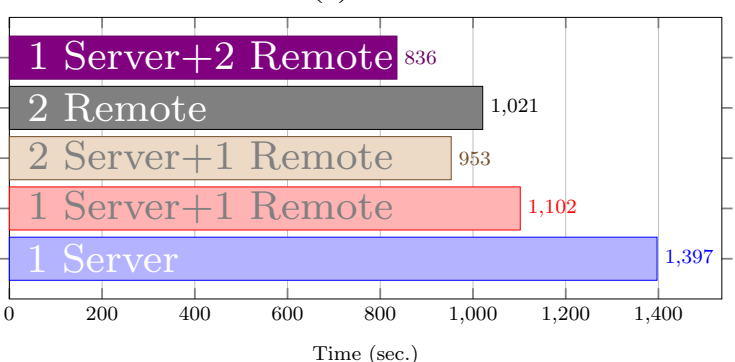

Fig. 25 a Result of Attilaexecution varying Sub-Contributors configurations on the same instance of the schema Reports consisting of $\sim 1.5 * 10^{5}$ rows (Server: 6 Core AMD Opteron(TM) $42843 \mathrm{GHz}$, 8GB, Remote: AMD Phenom(TM) II X6 1055T Processor $2.8 \mathrm{GHz}$,

As expected, we observed that performances improved when distributing the task among different machines.

Figure $25 \mathrm{~b}$ depicts the number of closed branches, which increases according to the size of the instance. These results confirm that our database instance is easier to evaluate than the artificial instances we built to prove NP-hardness results.

\section{Conclusions}

In this paper, we proposed a framework for discovering Approximate Pure Temporally Evolving Functional Dependencies (APE-FDs for short) from a temporal database. We have addressed in depth the data complexity of such problem. Unfortunately, this complexity turns out to be NP-Complete even for a single dependency. Moreover, moving to mining the set of $A P E$-FDs holding on an instance $\mathbf{r}$, the size of the result set depends also on the number of attributes of the schema of $\mathbf{r}$. For some instances, the lower bound of such size is exponential. We faced these problems in a real-world context, by proposing the use of model checking techniques, distributed computations and linear programming (b)

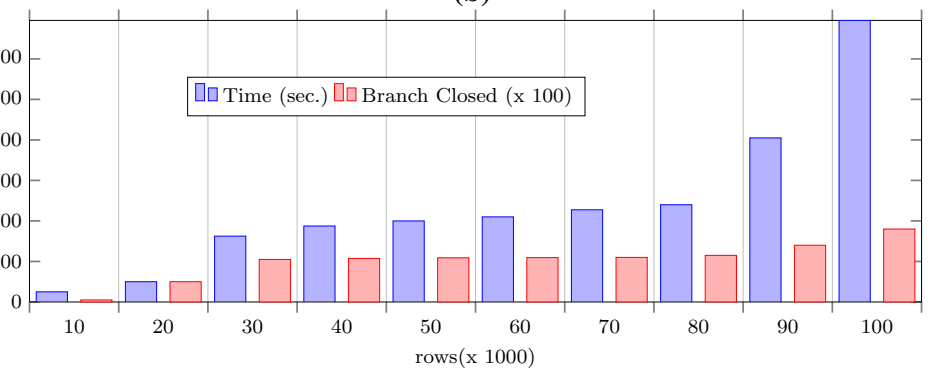

8GB). b Number of closed branches considering incremental portions of the same instance of schema Reports (the time refers to the execution on a Intel Core i3(TM) CPU M 330 2.13GHz, 4GB machine)

techniques. The implemented prototype Attila was tested on two real-world clinical scenarios and proved to be efficient. Moreover, we discussed the meaning of some interesting $A P E$-FDs mined from the databases in the psychiatry and pharmacovigilance domains, previously introduced. These results may provide (clinical) stakeholders with some new, previously unknown, understanding of the underlying data. We plan to further improve and extend our prototype, by integrating it in a platform allowing the discovery of different types of (temporal) approximate functional dependencies. Finally, we plan to perform an extended validation of mined $A P E$-FDs with clinical experts.

\section{Compliance with Ethical Standards}

Conflict of interest On behalf of all authors, the corresponding author states that there are no conflict of interest.

Ethical Approval This article does not contain any studies with human participants or animals performed by any of the authors.

Open Access This article is licensed under a Creative Commons Attribution 4.0 International License, which permits use, sharing, 
adaptation, distribution and reproduction in any medium or format, as long as you give appropriate credit to the original author(s) and the source, provide a link to the Creative Commons licence, and indicate if changes were made. The images or other third party material in this article are included in the article's Creative Commons licence, unless indicated otherwise in a credit line to the material. If material is not included in the article's Creative Commons licence and your intended use is not permitted by statutory regulation or exceeds the permitted use, you will need to obtain permission directly from the copyright holder. To view a copy of this licence, visit http://creativecommons .org/licenses/by/4.0/.

\section{References}

1. Bryant RE. Graph-based algorithms for Boolean function manipulation. IEEE Trans Comput. 1986;35(8):677-91. https://doi. org/10.1109/TC.1986.1676819.

2. Codd EF. Normalized data base structure: a brief tutorial. In: Proceedings of the 1971 ACM SIGFIDET (now SIGMOD) workshop on data description, access and control. ACM; 1971, pp. 1-17.

3. Combi C, Keravnou-Papailiou E, Shahar Y. Temporal information systems in medicine. Berlin: Springer; 2010.

4. Combi C, Mantovani M, Sabaini A, Sala P, Amaddeo F, Moretti U, Pozzi G. Mining approximate temporal functional dependencies with pure temporal grouping in clinical databases. Comput Biol Med. 2014;62:306-24.

5. Combi C, Mantovani M, Sala P. Discovering quantitative temporal functional dependencies on clinical data. In: 2017 IEEE international conference on healthcare informatics, ICHI 2017, Park City, UT, USA, Aug 23-26, 2017. IEEE Computer Society; 2017. pp. 248-257. https://doi.org/10.1109/ICHI.2017.80.

6. Combi C, Montanari A, Pozzi G. The T4SQL temporal query language. In: Silva MJ, Laender AHF, Baeza-Yates RA, McGuinness DL, Olstad B, Olsen ØH, Falcão AO, editors. CIKM. ACM; 2007. pp. 193-202.

7. Combi C, Montanari A, Sala P. A uniform framework for temporal functional dependencies with multiple granularities. In: Advances in spatial and temporal databases. Springer; 2011. pp. 404-421.

8. Combi C, Oliboni B, Pozzi G. Modeling and querying temporal semistructured data. In: New trends in data warehousing and data analysis. Springer; 2009. pp. 1-25.

9. Combi C, Pozzi G, Rossato R. Querying temporal clinical databases on granular trends. J Biomed Inform. 2012;45(2):273-91.

10. Combi C, Rizzi R, Sala P. The price of evolution in temporal databases. In: Grandi F, Lange M, Lomuscio A, editors, 22nd international symposium on temporal representation and reasoning, TIME 2015, Kassel, Germany, Sept 23-25, 2015. IEEE Computer Society; 2015. pp. 47-58. https://doi.org/10.1109/TIME.2015.24.

11. Combi C, Sala P. Mining approximate interval-based temporal dependencies. Acta Inf. 2016;53(6-8):547-85. https://doi. org/10.1007/s00236-015-0246-x.

12. Dvorský M. Common permutation problem. CoRR; 2008. arXiv :abs/0803.4261.

13. Huhtala Y, Karkkainen J, Porkka P, Toivonen H. Efficient discovery of functional and approximate dependencies using partitions. In: Proceedings of the 14th international conference on data engineering, 1998. IEEE; 1998. pp. 392-401.

14. Huhtala Y, Kärkkäinen J, Porkka P, Toivonen H. TANE: an efficient algorithm for discovering functional and approximate dependencies. Comput J. 1999;42(2):100-11.

15. Jensen CS, Snodgrass RT, Soo MD. Extending existing dependency theory to temporal databases. IEEE Trans Knowl Data Eng. 1996;8(4):563-82.
16. Kivinen J, Mannila H. Approximate inference of functional dependencies from relations. Theor Comput Sci. 1995;149(1):129-49.

17. Lind-Nielsen J. BuDDy-a binary decision diagram package. http://vlsicad.eecs.umich.edu/BK/Slots/cache/www.itu.dk/resea rch/buddy/. Accessed 3 Mar 2020.

18. Liu C, Zhang K, Xiong H, Jiang G, Yang Q. Temporal skeletonization on sequential data: patterns, categorization, and visualization. In: Proceedings of the 20th ACM SIGKDD international conference on Knowledge discovery and data mining. ACM; 2014. pp. 1336-1345.

19. Lopes S, Petit JM, Lakhal L. Functional and approximate dependency mining: database and fca points of view. J. Exp. Theor. Artif. Intell. 2002;14(2-3):93-114.

20. MedDRA. Medical Dictionary for Regulatory Activities. https ://www.meddra.org/. Accessed 23 Apr 2020.

21. Meyboom R, Lindquist M, Egberts A, Edwards I. Signal selection and follow-up in pharmacovigilance. Drug Saf. 2002;25(6):459-65.

22. Object Management Group: Business Process Model and Notation (BPMN), v2.0.2. http://www.omg.org/spec/BPMN/2.0.2/ PDF/. Accessed 3 Mar 2020.

23. Olson DL, Lauhoff G. Descriptive data mining. Computational risk management. 2nd ed. Berlin: Springer; 2019. https://doi. org/10.1007/978-981-13-7181-3.

24. Pryor J, Chinneck JW. Faster integer-feasibility in mixed-integer linear programs by branching to force change. Comput Oper Res. 2011;38(8):1143-52. https://doi.org/10.1016/j.cor.2010.10.025.

25. Sacchi L, Larizza C, Combi C, Bellazzi R. Data mining with temporal abstractions: learning rules from time series. Data Min Knowl Discov. 2007;15(2):217-47. https://doi.org/10.1007/s10618-007-0077-7.

26. Sala P. Approximate interval-based temporal dependencies: the complexity landscape. In: 2014 21st international symposium on temporal representation and reasoning (TIME). IEEE; 2014. pp. 69-78.

27. Sala P, Combi C, Cuccato M, Galvani A, Sabaini A. A framework for mining evolution rules and its application to the clinical domain. In: Balakrishnan P, Srivatsava J, Fu W, Harabagiu SM, Wang F, editors. 2015 International conference on healthcare informatics, ICHI 2015, Dallas, TX, USA, Oct 21-23, 2015. IEEE Computer Society; 2015. pp. 293-302. https://doi.org/10.1109/ ICHI.2015.42

28. Sordo M, Ochoa G, Murphy SN. A PSO/ACO approach to knowledge discovery in a pharmacovigilance context. In: GECCO (Companion); 2009. pp. 2679-2684.

29. Vaisman AA, Zimányi E. Data warehouse systems - design and implementation. Data-centric systems and applications. Berlin: Springer; 2014. https://doi.org/10.1007/978-3-642-54655-6.

30. Vianu V. Dynamic functional dependencies and database aging. J ACM (JACM). 1987;34(1):28-59.

31. Wang XS, Bettini C, Brodsky A, Jajodia S. Logical design for temporal databases with multiple granularities. ACM Trans. Database Syst. (TODS). 1997;22(2):115-70.

32. Wijsen J. Temporal FDs on complex objects. ACM Trans Database Syst. 1999;24(1):127-76.

33. Wijsen J. Temporal dependencies. In: Liu L, Özsu MT, editors. Encyclopedia of database systems. Berlin: Springer; 2009. p. 2960-6. https://doi.org/10.1007/978-0-387-39940-9_396.

34. World Health Organization and WHO Collaborating Centre for International Drug Monitoring: The Importance of Pharmacovigilance. Safety monitoring of medicinal products. World Health Organization; 2002.

Publisher's Note Springer Nature remains neutral with regard to jurisdictional claims in published maps and institutional affiliations. 